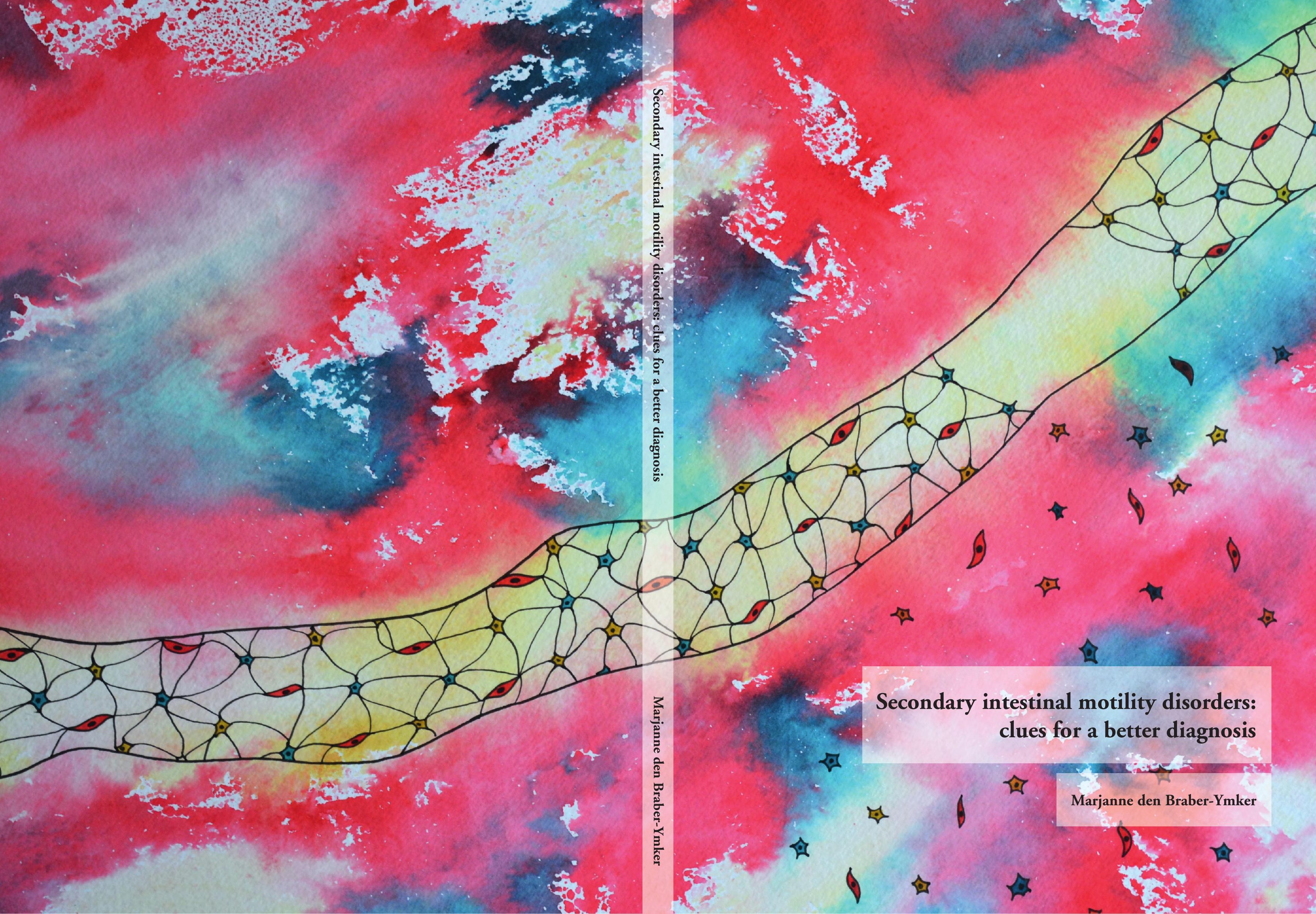




\section{SECONDARY INTESTINAL MOTILITY DISORDERS: CLUES FOR A BETTER DIAGNOSIS}

Marjanne den Braber-Ymker 
Dit proefschrift is goedgekeurd door:

de promotoren

prof. dr. ir. M.J.A.M. van Putten

prof. dr. I.D. Nagtegaal

de copromotor

prof. dr. M.M.Y. Lammens

Cover design: Erna Ymker

Layout: ProefschriftOntwerp

Printing: ProefschriftMaken

ISBN: 978-90-365-4833-5

DOI: $10.3990 / 1.9789036548335$

(C) 2019, M. den Braber-Ymker, The Netherlands.

All rights reserved. No parts of this thesis may be reproduced, stored in a retrieval system or transmitted in any form or by any means without permission of the author.

Alle rechten voorbehouden. Niets uit deze uitgave mag worden vermenigvuldigd, in enige vorm of op enige wijze, zonder voorafgaande schriftelijke toestemming van de auteur. 


\title{
SECONDARY INTESTINAL MOTILITY DISORDERS: CLUES FOR A BETTER DIAGNOSIS
}

\section{PROEFSCHRIFT}

\author{
ter verkrijging van \\ de graad van doctor aan de Universiteit Twente, \\ op gezag van de rector magnificus, \\ prof. dr. T.T.M. Palstra, \\ volgens besluit van het College voor Promoties \\ in het openbaar te verdedigen \\ op 31 oktober 2019 om 12.45 uur
}

door

\section{Marjanne den Braber-Ymker}

geboren op 6 mei 1986

te Zweeloo, Nederland 


\section{Promotiecommissie:}

\section{Voorzitter/secretaris}

prof. dr. J.L. Herek

Universiteit Twente

Promotoren

prof. dr. ir. M.J.A.M. van Putten Universiteit Twente

prof. dr. I.D. Nagtegaal Radboudumc

\section{Copromotor}

prof. dr. M.M.Y. Lammens

Universitair Ziekenhuis Antwerpen

\section{Leden}

prof. dr. ir. C.H. Slump

Universiteit Twente

prof. dr. M.M.A.E. Claessens

Universiteit Twente

prof. dr. I. de Blaauw

Radboudumc

prof. dr. J.J. Kolkman

Universitair Medisch Centrum Groningen

dr. P.M.A. Broens

Universitair Medisch Centrum Groningen

dr. R.S. van der Post

Radboudumc 




\section{Contents}

Chapter 1 General introduction and outline of the thesis

Part I Methodological issues

Chapter 2 Practical and reproducible estimation of myenteric interstitial

Chapter 3 Semiquantitative methods

Chapter 4 The enteric nervous system and the musculature of the colon are altered in patients with spina bifida and spinal cord injury

Chapter 5 Intestinal involvement in amyloidosis is a sequential process

Chapter 6 Intestinal hypomotility in systemic sclerosis: a histological study into the sequence of events

Chapter 7 General discussion

$\begin{array}{lll}\text { Appendices } & \text { Summary } & 131\end{array}$

Nederlandse samenvatting $\quad 137$

List of abbreviations $\quad 143$

List of publications, abstracts and presentations $\quad 145$

Curriculum Vitae $\quad 147$

Dankwoord 149 


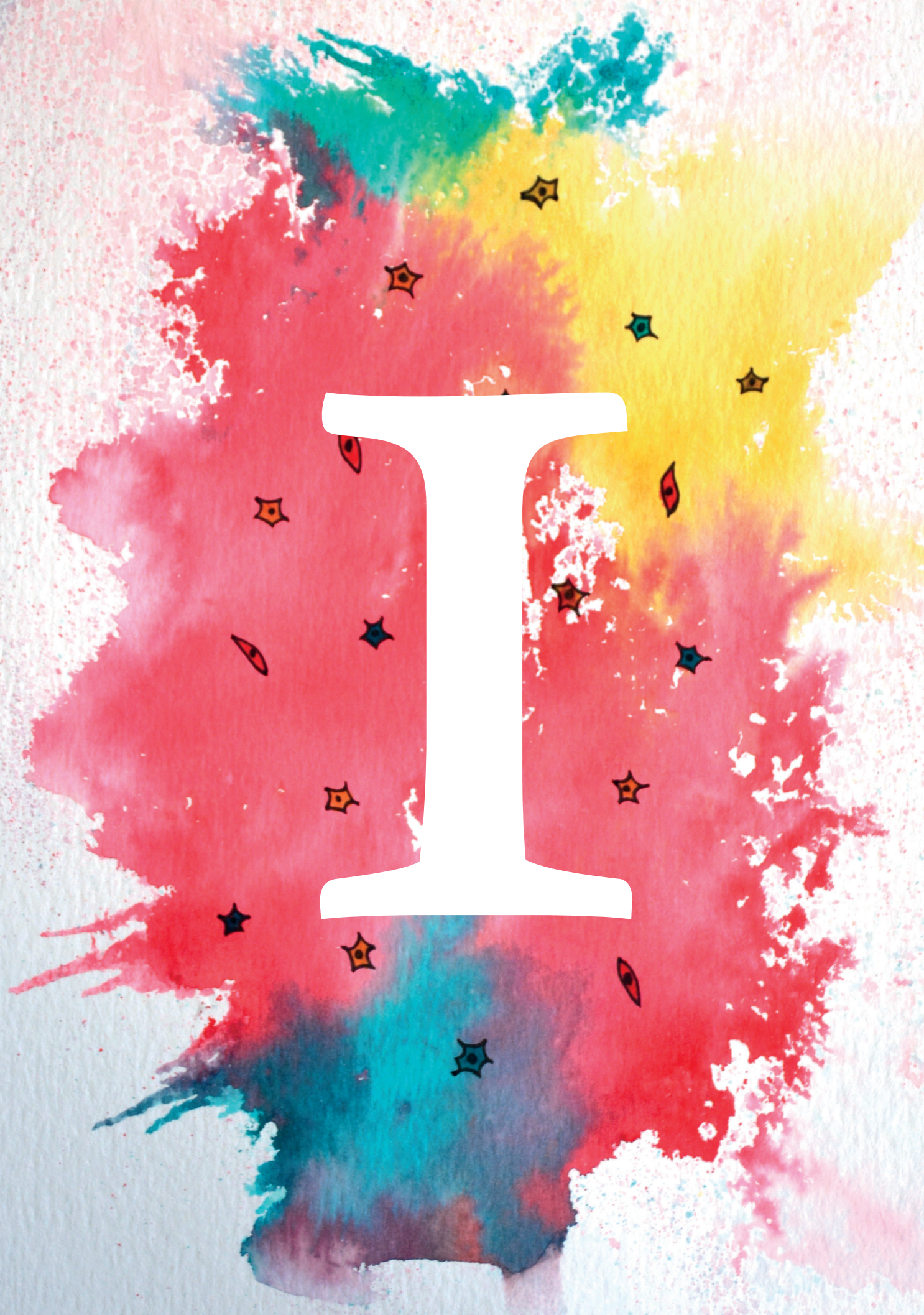




\section{Chapter I}

General introduction 
Chapter I 


\section{General introduction and outline of the thesis}

The digestive system is an essential organ system in the body and frequently involved in a broad spectrum of diseases, such as cancers, inflammatory diseases and neuromuscular disorders. The latter is the least well-known group of diseases, which is characterised by gastrointestinal dysmotility.

\section{Anatomy and physiology of the intestines}

The intestines are part of the gastrointestinal (GI) tract. The small intestine extends from the pylorus to the ileocaecal junction. The small intestine is approximately 6 to 7 metres long and consists of the duodenum, jejunum and ileum (Figure 1.1A). The large intestine extends from the distal end of the ileum to the anus and is 1 to 1.5 metres long in adults. Parts of the large intestine are caecum, appendix, colon, rectum and anal canal (Figure 1.1B). The colon consists of the ascending, transverse, descending and sigmoid colon. $^{[1]}$

Digestion and absorption of dietary nutrients are the main functions of the small intestines, and its motor activity is closely related to these functions. Small intestinal motility during feeding is characterised by segmental contractions that churn the bowel contents and peristaltic contractions resulting in a forward propulsive motion of luminal contents. In the fasting state, migrating motor complexes are responsible for slow rhythmic contractions to clear the gut lumen. ${ }^{[2]}$ Functions of the large bowel are absorption of water, electrolytes and short-chain fatty acids, storage and elimination of faeces. The colon has two types of motor activity: nonpropulsive segmentation generated by slow wave activity that churns the bowel content and mass peristalsis that propels the content distally. ${ }^{[2]}$ 

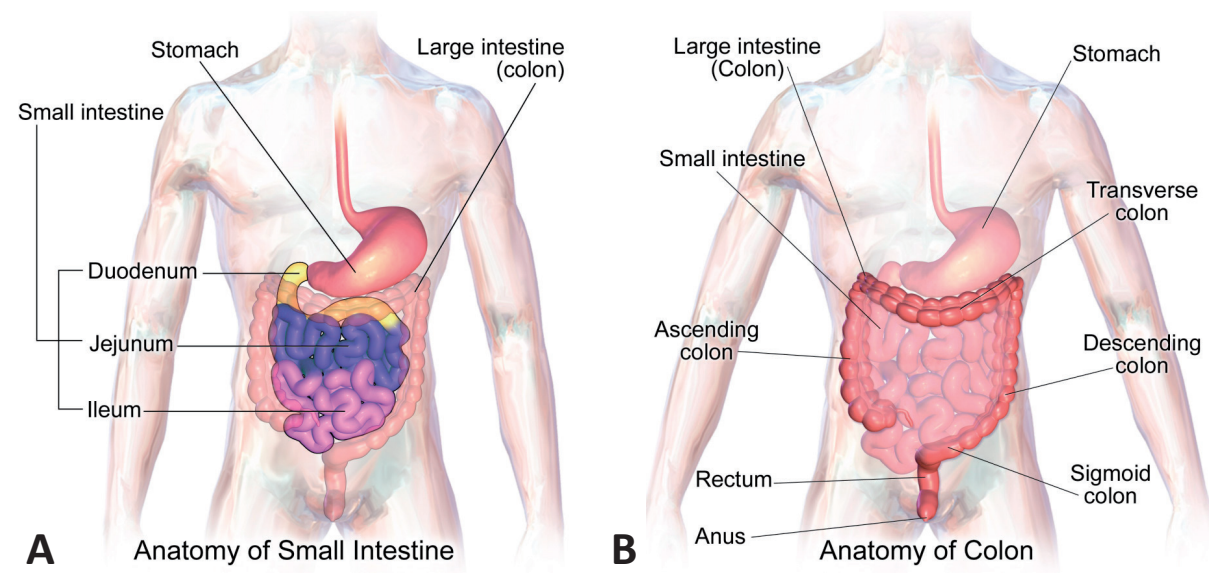

Figure I.I Anatomy of the small intestine (A) and colon (B). ${ }^{[3]}$

\section{The intestinal wall}

The gut wall is generally similar throughout its length and consists of several layers: mucosa, submucosa, muscularis propria (or externa) and serosa (Figure 1.2). The mucosa consists of an epithelial layer, connective tissue forming the lamina propria and a thin muscular layer called the muscularis mucosae. The surface for absorption in the small intestine is largely increased by the presence of circular folds (plicae circulares), villi and microvilli, while the mucosa of the colon has deep crypts but no villi. Epithelial cells of the colon contain microvilli as well. The submucosa contains loose connective tissue with the submucosal (or Meissner's) plexus, blood and lymphatic vessels. The muscularis propria includes two muscle layers: the inner layer with circumferentially oriented smooth muscle fibres (circular layer) and the outer layer containing longitudinally oriented muscle fibres (longitudinal layer). The myenteric (or Auerbach's) plexus is situated between the circular and longitudinal muscle layer. The serosa is a thin connective tissue layer surrounding the muscularis propria. ${ }^{[4]}$ 

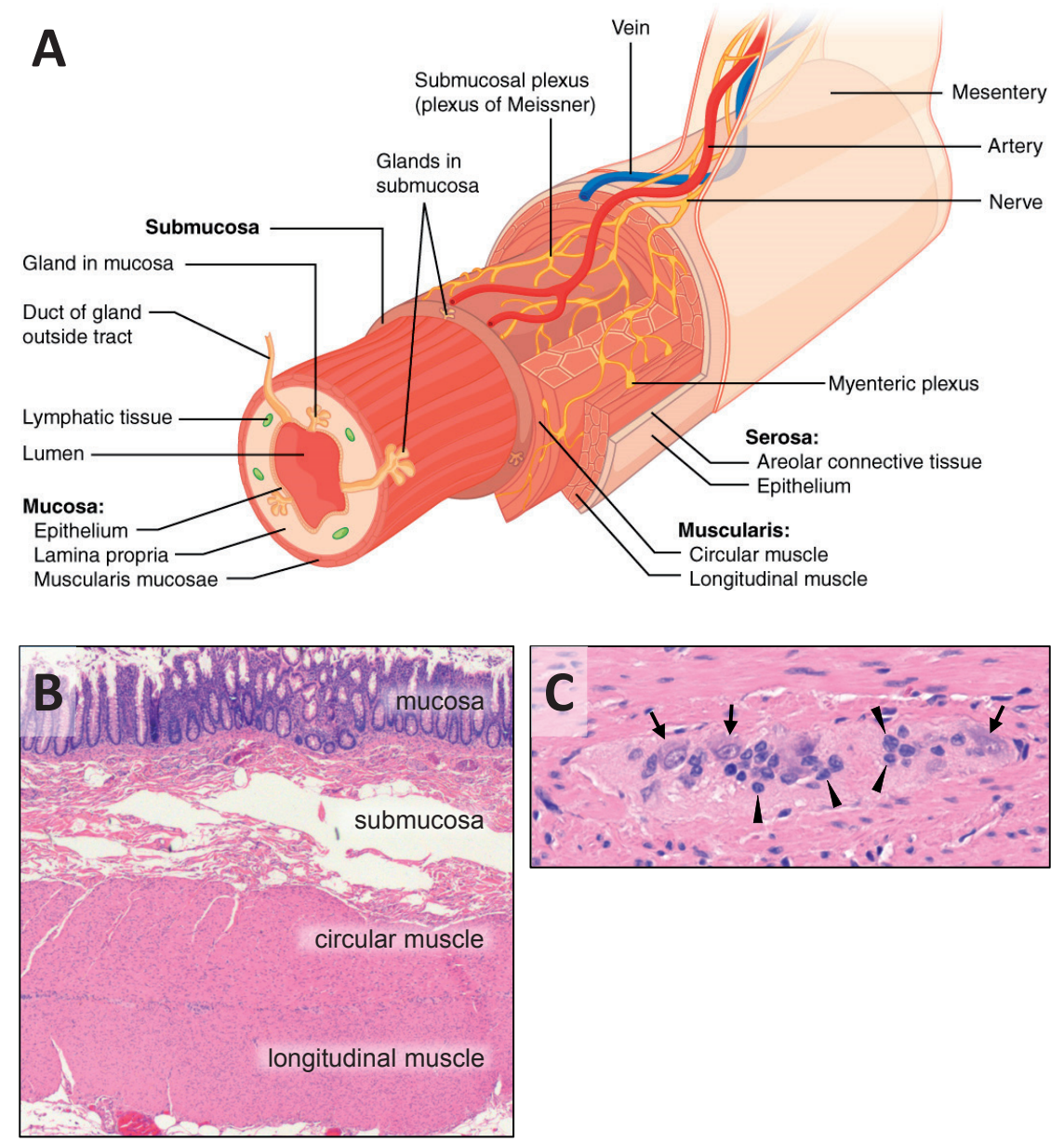

Figure I.2 Anatomy of the intestinal wall.

A) The gut wall consists of mucosa, submucosa, muscularis propria and serosa. The submucosal and myenteric plexuses are part of the enteric nervous system and are situated in the submucosa and between the circular and longitudinal muscle layer of the muscularis propria, respectively. B) Histology of the gut wall shows the different layers, H\&E stain. C) Ganglion of the myenteric plexus containing neurons (arrows) and glial cells (arrow heads). Image A obtained from Wikimedia Commons under the Creative Commons Attribution-Share Alike 3.0 Unreported license. ${ }^{[5]}$ 


\section{Gut motility}

Intestinal motility (segmentation and peristalsis) is mainly regulated by the neuromuscular apparatus of the bowel. The neuromuscular apparatus of the gut consists of the enteric nervous system (ENS), the interstitial cells of Cajal (ICCs) and the smooth muscle cells of the muscularis propria. ${ }^{[6]}$ The muscularis mucosae is usually not included in the neuromuscular system ${ }^{[6,7]}$, because its main function is movement of the mucosa and villi. Intestinal function is controlled by both intrinsic and extrinsic innervation. The ENS forms the intrinsic nervous system and is the primary mechanism that controls motility and secretion. The ENS consists of approximately 100 million neurons along the whole GI tract, that functions largely independently of the central nervous system, but can be modified by extrinsic input from the parasympathetic and sympathetic nerves (Figure 1.3). ${ }^{[2,8]}$ The small intestine and proximal two-thirds of the colon receive signals from the distal branches of the vagal nerve, the distal third of the colon and the rectum are innervated by the pelvic nerves. Stimulation of parasympathetic efferent fibres results in increased secretion and motility. Parasympathetic nerves also contain afferent fibres, transporting sensory information to the medulla oblongata from chemoreceptors and mechanoreceptors in the mucosa. Sympathetic nerve fibres leave the spinal cord in the thoracolumbar region and synapse with neurons in the prevertebral sympathetic ganglia located in the abdomen: the caeliac, superior mesenteric and inferior mesenteric ganglia. These neurons project on neurons in the ENS or directly innervate effector cells. Stimulation of sympathetic efferent fibres results in suppression of gut activity. ${ }^{[2,9]}$

The submucosal plexus and myenteric plexus form the histological recognisable part of the ENS, which consist of a network of interconnecting ganglia. ${ }^{[4]}$ The ganglia contain clusters of neurons (ganglion cells), enteric glial cells and neural processes. The neurons can be identified on haematoxylin and eosin (H\&E) stained sections by their large oval shape, pink cytoplasm with basophilic Nissl substance, and a large nucleus with single prominent nucleolus (Figure 1.2C) ${ }^{[4]}$ Enteric glial cells support the neurons in the ganglia and ensheath the neuronal processes. Glial cells are increasingly recognised as important for regulatory functions in the gut, including the control of motility. ${ }^{[10,11]}$ Enteric glial cells were recognised as a unique class of peripheral glia in the early 1970s. ${ }^{[12,13]}$ Enteric glia show more similarities with astrocytes in the central nervous system (e.g. star-shape, similar gliofilaments) than with Schwann cells in the peripheral nervous system. However, enteric glia and astrocytes have different developmental origins, indicating that enteric glia are fundamentally different from astrocytes. ${ }^{[12]}$ Today, at least three subpopulations of enteric glial cells are identified in the intestinal wall: mucosal, intraganglionic and intramuscular glia, which are found in the lamina propria, inside the plexus, and in the layers of the muscularis propria, respectively. Mucosal glia are located directly below the epithelium and interact with enteroendocrine cells (sensory function), nerve fibres and blood vessels in the mucosa. In the submucosal and myenteric 
ganglia, glial cells are closely related to the neurons: a subset of intraganglionic glial cells are connected by gap junctions. Intramuscular glia are long and bipolar shaped and are associated with nerve fibres that innervate the smooth muscle cells. ${ }^{[14]}$ The ENS is responsible for the control of gut motility by intrinsic reflex circuits. These reflexes are initiated by afferent sensory neurons, which are sensitive to chemical and mechanical changes and connect with interneurons. Interneurons activate efferent motor neurons, which stimulate or inhibit various effector cells, including ICCs and smooth muscle cells. ${ }^{[2,15,16]}$ Mucosal secretion and blood flow are mainly regulated by the submucosal plexus, while gut motility is primarily controlled by the myenteric plexus. ${ }^{[17,18]}$

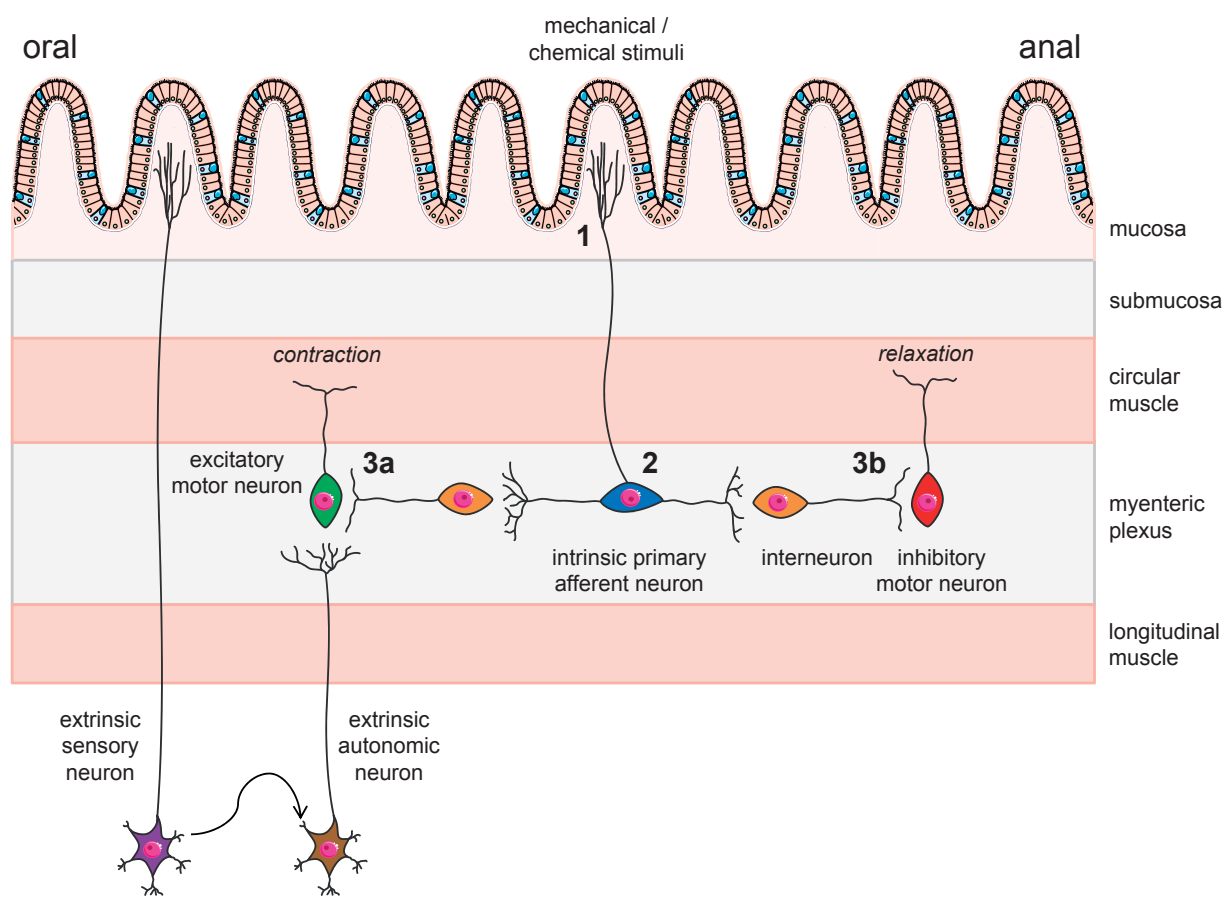

Figure I.3 Innervation of the gut wall.

Extrinsic sensory neurons (purple) activate parasympathetic and sympathetic nerves (brown) that may stimulate or inhibit the enteric neurons in the intrinsic nervous circuit. The peristaltic reflex works as follows: 1) mechanical or chemical stimuli activate intrinsic primary afferent neurons (IPANs, blue); 2) IPANs activate interneurons (orange); 3a) ascending interneurons (oral direction) activate excitatory motor neurons (green) that cause smooth muscle contraction; 3b) descending interneurons (anal direction) activate inhibitory motor neurons (red) that result in smooth muscle relaxation. This schematic illustration is not drawn to scale. This figure is created with images adapted from Servier Medical $\operatorname{Art}^{[19]}$. Original images are licensed under a Creative Commons Attribution 3.0 Unreported License. Simplification and colour changes were made to the original neuron and mucosa cartoons. 
ICC networks are found in several layers of the bowel wall, variable per region in the GI tract. ${ }^{[20-22]}$ ICCs are pacemaker cells that generate and propagate electrical slow waves to smooth muscle cells. ${ }^{[20]}$ They also play a role in neurotransmission and may function as stretch receptor. ${ }^{[16,22]}$ Most ICCs are present around the circumference of the myenteric plexus, forming a mesh-like network. ${ }^{[21]}$ The myenteric ICC network may play a major role in peristalsis, while the submucosal ICC network may generate slow waves to enhance absorption. ${ }^{[23]}$ Intramuscular ICCs may play an intermediary role in enteric neuromuscular transmission and as mechanoreceptors. ${ }^{[22]}$

Smooth muscle cells are activated by enteric neurons and ICCs and are therefore the final effector cells of gut motility. Gap junctions provide connections between smooth muscle cells to facilitate coordinated contractions in the bowel. ${ }^{[17]}$

\section{Gastrointestinal neuromuscular diseases}

Abnormalities in one or more components of the neuromuscular apparatus of the GI tract (ENS, ICC and muscularis propria) can result in disturbance of gut motility. The term gastrointestinal neuromuscular disease (GINMD) describes a clinically heterogeneous group of chronic diseases characterised by GI dysmotility, as a result of neuromuscular dysfunction and/or interactions with other cells such as those of the immune system. ${ }^{[24,25]}$ Neuromuscular disorders can be either congenital (e.g. Hirschsprung's disease) or acquired (e.g. intestinal pseudo-obstruction and slow transit constipation). ${ }^{[25]}$ The term GINMD includes both primary motility disorders (e.g. Hirschsprung's disease, chronic intestinal pseudo-obstruction, slow transit constipation), in which abnormalities of the enteric neuromusculature is the primary cause of disease, and secondary motility disorders, as result of a systemic disease (e.g. systemic sclerosis, amyloidosis). ${ }^{[7]}$

GINMDs can be defined based on clinical, radiological, physiological or histological criteria, which make the definition of such disorders more complex than it may first appear. Diseases are categorised as either symptom-based (e.g. inflammatory bowel syndrome, functional dyspepsia, idiopathic constipation) or measurement based (e.g. enteric dysmotility, intestinal pseudo-obstruction, slow transit constipation). ${ }^{[25]}$ Approximately one third of the general population in Western countries have unexplained symptoms related to the GI tract, which are often termed 'functional' or 'idiopathic'. Although most of these patients have mild to moderate symptoms, a minority of patients suffer from severe symptoms, including chronic pain, nausea, vomiting, bloating and severe constipation. ${ }^{[26]}$ GNIMDs show thus different degrees of dysmotility and symptoms have a variable negative impact on patients' quality of life. ${ }^{[6]}$ Some patients have recurrent episodes of pseudo-obstruction, characterised by clinical symptoms of intestinal obstruction but without any mechanical lesion in the gut lumen. ${ }^{[27]}$ However, increasing evidence suggests that gut dysmotility in 'functional' or 'idiopathic' GI 
disorders may have underlying distinct histopathological abnormalities (i.e. mucosal, neuroenteric, muscular) ${ }^{[6]}$ To create consensus about the histopathological approach of this complex disease group, the Gastro 2009 International Working Group published guidelines for histological analysis and diagnosis of gastrointestinal neuromuscular pathology (GINMP). ${ }^{[24]}$ These guidelines were followed by the London Classification, providing a first classification of diagnostic criteria for histopathological phenotypes in relation to entities observed in clinical practice. ${ }^{[7]}$ Generally, GINMP can be divided into neuropathies, myopathies and ICC abnormalities (enteric mesenchymopathy). ${ }^{[7]}$ The International Working Group believes that accurate histopathological reporting will in the future result in better understanding of disease mechanisms, developments of diagnostic biomarkers and possibly more effective targeted therapies. ${ }^{[7,24]}$ In the future, terms as 'functional' or 'idiopathic' may be replaced by more suitable definitions such as enteric neuro-gliopathies or neuro-myopathies. ${ }^{[6,11]}$

A careful histological analysis of gut tissues is thus important to support the clinical diagnosis of GINMDs. ${ }^{[6,28]}$ Full-thickness biopsies are required for systematic examination of the neuromuscular apparatus of the gut. ${ }^{[24,28]}$ Furthermore, use of a panel of histochemical and immunohistochemical stains is helpful in this evaluation. ${ }^{[6,24,28,29]}$

\section{Aim and outline}

The pathophysiologic disease mechanisms in GINMDs are complex and only in a minority of diseases the pathogenesis is well understood, including Hirschsprung's disease, mitochondropathies and Chagas disease. ${ }^{[25]}$ Primary GINMDs functionally defined as chronic intestinal pseudo-obstruction and slow transit constipation show a large individual variation for underlying mechanisms. For example, some patients with chronic intestinal pseudo-obstruction show neuropathic changes, while others have myopathic changes of the bowel wall or a combination of both. ${ }^{[7]}$ Consequently, a wide range of histological characteristics in the bowel have been described for chronic intestinal pseudo-obstruction, which makes it impossible to distinguish one general underlying pathophysiological mechanism. ${ }^{[30]}$ The aetiology of secondary motility disorders is generally better understood than that of primary GINMDs, because of our knowledge of the primary disease. However, detailed pathophysiological mechanisms leading to intestinal dysmotility in those patients are less clear. Systematic histological evaluation of the neuromuscular structures in the bowel wall of these patients can explain their clinical symptoms of intestinal dysmotility. The aim of this thesis is to investigate the histological background of the pathophysiology of secondary gastrointestinal neuromuscular diseases with a clear aetiology (spinal cord damage, amyloidosis and systemic sclerosis). Correlation of aetiology with histopathology may help to understand potential pathological mechanisms of intestinal motility disorders in general. 
The first part of this thesis describes how histopathological characteristics are studied in a systematic way using semiquantitative scoring methods. The second part of this thesis includes the research into the histological background of the secondary motility disorders using full-thickness patient tissue material from autopsies or surgical resections to assess the neuromuscular structures in the bowel wall. Neuromuscular structures that are histologically studied in this thesis are the submucosal and myenteric plexus (neurons and glial cells), the myenteric ICC network, and the muscularis propria.

\section{Part I: Methodological issues}

Chapter 2 describes a semiquantitative method for estimation of the ICC network around the circumference of the myenteric plexus, which is easy to use in clinical diagnostic setting.

- Chapter 3 explains how the ENS and muscular layers of the bowel wall are assessed in a semiquantitative way. These semiquantitative methods are used in the next chapters.

\section{Part II: Secondary motility disorders}

- Chapter 4 aims to correlate histological changes in the bowel wall to intestinal dysmotility in patients with spina bifida and spinal cord injury.

- Chapter 5 explores histological characteristics of the bowel wall in AL and AA amyloidosis to gain more insight in pathophysiological mechanisms associated with intestinal dysmotility in amyloidosis.

- Chapter 6 aims to investigate histopathological features of the intestinal wall which may be associated with hypomotility in systemic sclerosis.

The final chapter discusses the key findings of this thesis and gives future perspectives. 


\section{References}

1. Moore KL, Dalley AF, Agur AMR. Clinically Oriented Anatomy. 8th ed. 2017: Wolters Kluwer Health/Lippincott Williams \& Wilkins.

2. Boron WF, Boulpaep EL. Medical physiology: a cellular and molecular approach. Second ed. 2012, Philadelphia: Elsevier Health Sciences.

3. Blausen.com staff (2014). Medical gallery of Blausen Medical 2014. WikiJournal of Medicine 1(2). DOI: $10.15347 / \mathrm{wjm} / 2014.010$. ISSN 2002-4436.

4. Mills SE. Histology for Pathologists. 2007: Lippincott Williams \& Wilkins.

5. OpenStax College, Rice University (2013). Layers of the Gastrointestinal Tract. Connexions, OpenStax College, Anatomy \& Physiology, Overview of the Digestive System.

6. Bernardini N, Ippolito C, Segnani C, et al. Histopathology in gastrointestinal neuromuscular diseases: methodological and ontological issues. Adv Anat Pathol, 2013. 20(1): p. 17-31.

7. Knowles $\mathrm{CH}$, De Giorgio R, Kapur RP, et al. The London Classification of gastrointestinal neuromuscular pathology: report on behalf of the Gastro 2009 International Working Group. Gut, 2010. 59(7): p. 882-887.

8. Camilleri M, Ford MJ. Review article: colonic sensorimotor physiology in health, and its alteration in constipation and diarrhoeal disorders. Aliment Pharmacol Ther, 1998. 12(4): p. 287-302.

9. Rhoades RA, Bell DR. Medical Physiology: Principles for Clinical Medicine. 2012: Wolters Kluwer Health/Lippincott Williams \& Wilkins.

10. Sharkey KA. Emerging roles for enteric glia in gastrointestinal disorders. J Clin Invest, 2015. 125(3): p. 918-925.

11. Bassotti G, Villanacci V. Can "functional" constipation be considered as a form of enteric neurogliopathy? Glia, 2011. 59(3): p. 345-350.

12. Gulbransen BD. Enteric Glia. 2014: Biota Publishing.

13. Gabella G. Fine structure of the myenteric plexus in the guinea-pig ileum. J Anat, 1972. 111(Pt 1): p. 69-97.

14. Rao M, Gershon MD. Enteric nervous system development: what could possibly go wrong? Nat Rev Neurosci, 2018. 19(9): p. 552-565.

15. Costa M, Brookes SJ, Hennig GW. Anatomy and physiology of the enteric nervous system. Gut, 2000. 47 Suppl 4: p. iv15-19; discussion iv26.

16. Johnson L, Ghishan F, Kaunitz J, et al. Physiology of the Gastrointestinal Tract. Physiology of the Gastrointestinal Tract. Vol. 1-2. 2012.

17. Furness JB. The Enteric Nervous System. Second ed. 2008, Oxford: Wiley-Blackwell.

18. Wood JD. Enteric Nervous System: Physiology, in Encyclopedia of Neuroscience, Squire LR, Editor. 2009, Academic Press: Oxford. p. 1103-1113.

19. Servier Medical Art. Intestinal villi; Neuron. Free medical images [Internet]. Les Laboratoires Servier. Available from: https://smart.servier.com/. Accessed 2019 April 17.

20. Sanders KM, Ward SM, Koh SD. Interstitial cells: regulators of smooth muscle function. Physiol Rev, 2014. 94(3): p. 859-907. 


\section{Chapter I}

21. Al-Shboul OA. The importance of interstitial cells of cajal in the gastrointestinal tract. Saudi J Gastroenterol, 2013. 19(1): p. 3-15.

22. Komuro T. Atlas of Interstitial Cells of Cajal in the Gastrointestinal Tract. 2012, Dordrecht: Springer Science \& Business Media.

23. Huizinga JD, Martz S, Gil V, et al. Two independent networks of interstitial cells of cajal work cooperatively with the enteric nervous system to create colonic motor patterns. Front Neurosci, 2011. 5: p. 93.

24. Knowles CH, De Giorgio R, Kapur RP, et al. Gastrointestinal neuromuscular pathology: guidelines for histological techniques and reporting on behalf of the Gastro 2009 International Working Group. Acta Neuropathol, 2009. 118(2): p. 271-301.

25. Knowles $\mathrm{CH}$. New horizons in the pathogenesis of gastrointestinal neuromuscular disease. J Pediatr Gastroenterol Nutr, 2007. 45 Suppl 2: p. S97-102.

26. Knowles CH, Lindberg G, Panza E, et al. New perspectives in the diagnosis and management of enteric neuropathies. Nat Rev Gastroenterol Hepatol, 2013. 10(4): p. 206-218.

27. Mann SD, Debinski HS, Kamm MA. Clinical characteristics of chronic idiopathic intestinal pseudo-obstruction in adults. Gut, 1997. 41(5): p. 675-681.

28. Odze RD, Goldblum JR. Surgical Pathology of the GI Tract, Liver, Biliary Tract and Pancreas. 2014: Elsevier Health Sciences.

29. Bassotti G, Villanacci V, Salerni B, et al. Beyond hematoxylin and eosin: the importance of immunohistochemical techniques for evaluating surgically resected constipated patients. Tech Coloproctol, 2011. 15(4): p. 371-375.

30. Downes TJ, Cheruvu MS, Karunaratne TB, et al. Pathophysiology, Diagnosis, and Management of Chronic Intestinal Pseudo-Obstruction. J Clin Gastroenterol, 2018. 52(6): p. 477-489. 
General introduction and outline of the thesis 


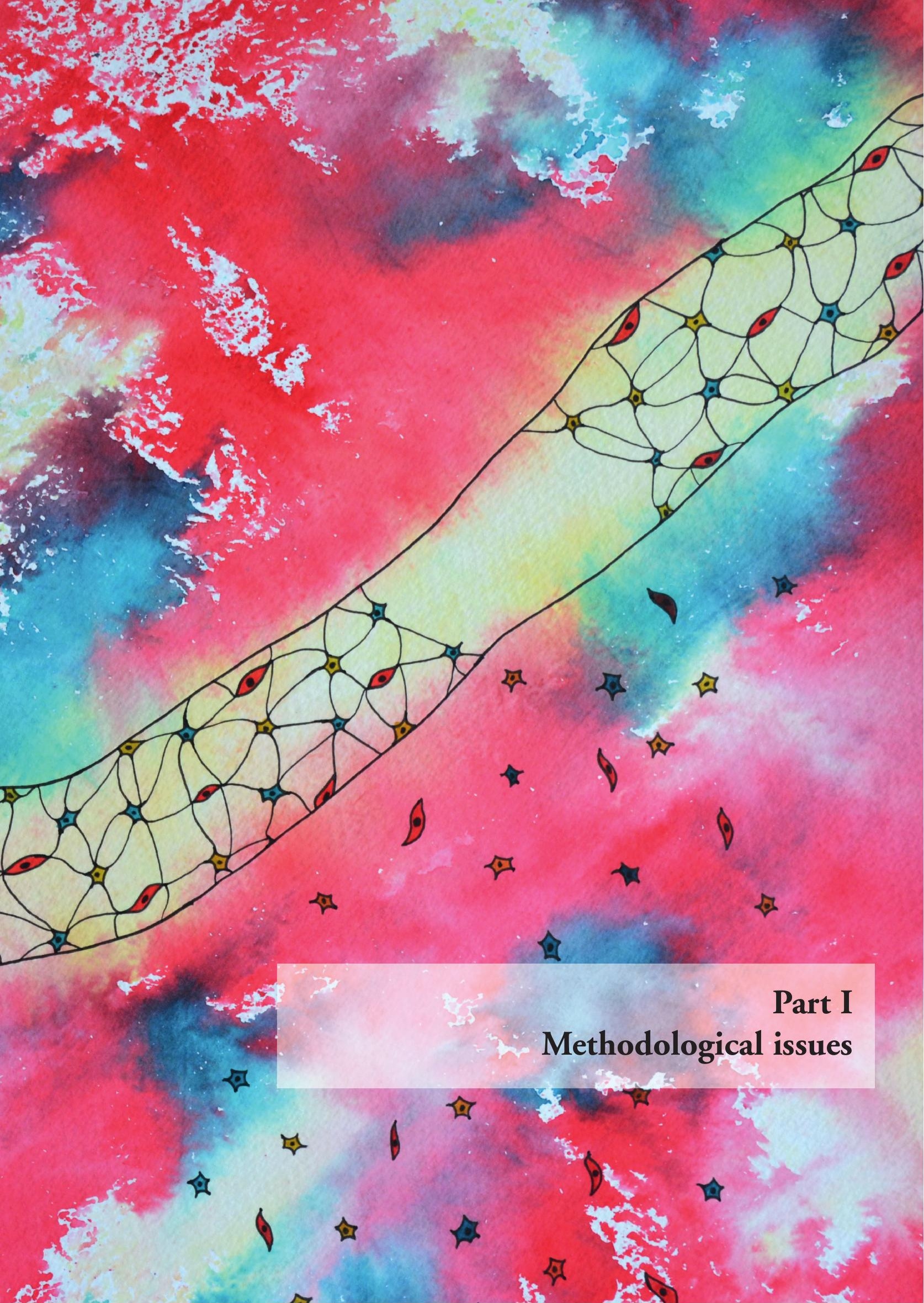




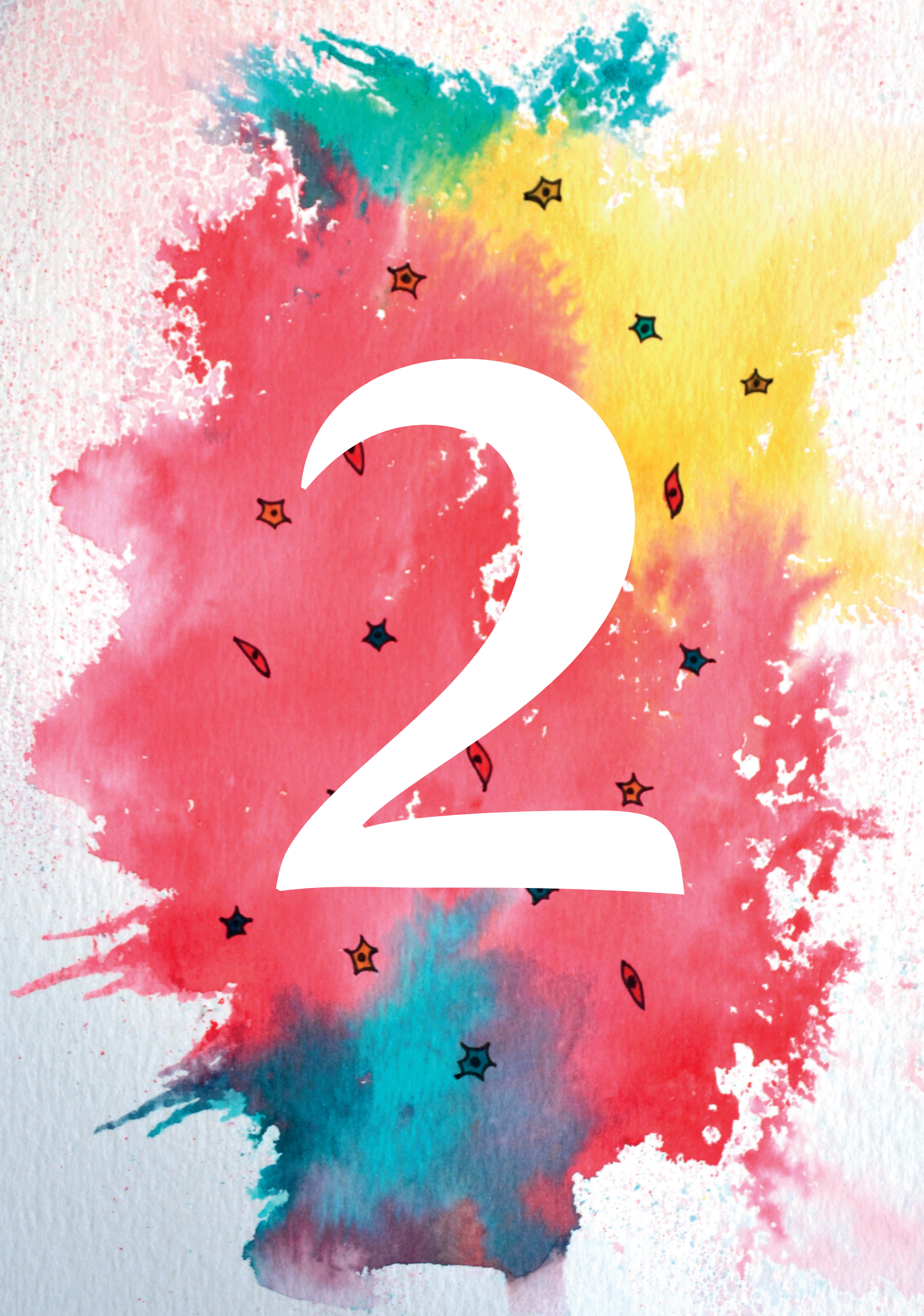




\title{
Chapter 2
}

\section{Practical and reproducible estimation of myenteric interstitial cells of Cajal in the bowel for diagnostic purposes}

\author{
M. den Braber-Ymker ${ }^{1}$, S. Heijker ${ }^{1}$, M. Lammens ${ }^{1,2,3}$, I. D. Nagtegaal ${ }^{1}$ \\ ${ }^{1}$ Department of Pathology, Radboud University Medical Center, Nijmegen, The Netherlands \\ ${ }^{2}$ Department of Pathology, Antwerp University Hospital, University of Antwerp, Edegem, Belgium \\ ${ }^{3}$ MIPRO, University of Antwerp, Antwerp, Belgium
}

Neurogastroenterol Motil, 2016; 28(8): 1261-1267 


\section{Abstract}

Histological assessment of the interstitial cells of Cajal (ICCs) in the bowel is important for diagnosing patients with gastrointestinal neuromuscular diseases (GINMD). Although the International Working Group on GINMD proposed reporting a decrease in ICC number of more than 50\%, quantitative methods used in literature are not practical for daily routine of the pathologist. Consequently, this study presents a straightforward semiquantitative estimation method for myenteric ICCs of the bowel.

Formalin-fixed paraffin-embedded sections from small bowel $(\mathrm{n}=87)$ and colon $(n=159)$ were collected to create two control groups and four groups composed of patients with gastrointestinal motility disorders. The control groups included material of resection and autopsy origin, respectively. Samples were stained with CD117 (c-kit) antibody to estimate the myenteric ICC network. Scores of two observers were compared to analyse inter- and intraobserver agreement and reliability.

Interobserver reliability was almost perfect for small bowel (intraclass correlation coefficient 0.847 ; 95\% confidence interval (CI) (0.774-0.897)) and substantial for colon $(0.683 ; 95 \%$ CI $(0.591-0.758))$. Almost perfect intraobserver reliability was found (intraclass correlation coefficient 0.918 ; 95\% CI (0.874-0.947)). The small bowel showed more myenteric ICCs than the colon. No significant differences between colonic regions were found, nor were there any differences in the orientation of the sections.

The proposed estimation method for the myenteric ICC network showed generally good agreement and reliability. Since the method is semiquantitative, simple and capable to differentiate between normal and diseased tissue, it can be used in routine diagnostics of gastrointestinal neuromuscular disorders. 


\section{Introduction}

The neuromuscular apparatus of the gut includes the enteric nervous system (ENS), the interstitial cells of Cajal (ICCs) and the smooth muscle of the muscularis propria. Changes in these structures may result in gastrointestinal neuromuscular disease (GINMD). Histological evaluation is important for an appropriate diagnosis. ${ }^{[1]}$ This study focuses on the ICC network around the circumference of the myenteric plexus. ICCs in the bowel wall are the pacemaker cells that generate slow waves to the smooth muscle cells, but they also play a role in neurotransmission and function as stretch receptor. ${ }^{[2]}$ Therefore, absence or disruption of the ICC network can result in motility disorders. ${ }^{[3]}$ Indeed, many gastrointestinal neuromuscular diseases are associated with ICC loss. ${ }^{[3]}$ Consequently, histological evaluation of ICCs in intestinal tissue in these patients is important. The International Working Group on GINMD suggested that only a reduction of more than $50 \%$ of ICCs should be reported, because of the wide variation in normal values. ${ }^{[1]}$ Several quantification methods are described in literature, including counting of ICCs by a grid, using high-power fields or confocal microscopy. ${ }^{[4-6]}$ However, these methods are time-consuming and therefore not useful in the daily routine of the pathologist. Hence, pathologists assess the presence of ICCs by rough estimation, based on their own experiences.

ICC networks are found in several layers of the intestinal wall, although some networks are more important for motility than others. ${ }^{[2,3]}$ Huizinga et al ${ }^{[7]}$ hypothesised that the myenteric ICC network is involved in propulsion, while the submucosal ICC network generate slow waves to enhance absorption. Thus, the myenteric network may play a major role in peristalsis. ${ }^{[8]}$ This network is formed around the circumference of the myenteric plexus between circular and longitudinal smooth muscle layers of the intestinal wall. ${ }^{[8,9]}$

This study proposed a novel semiquantitative estimation method for ICCs surrounding the intestinal myenteric plexus, which could easily be implemented in diagnostic practice.

\section{Materials and Methods}

\section{Subjects}

For our study we collected two control groups of patients without gastrointestinal motility disorders and four case groups, that are composed of patients with a variety of gastrointestinal motility disorders. The first control group included archived formalinfixed paraffin-embedded (FFPE) segments of ileum and colon, obtained from patients who underwent right-sided hemicolectomy for non-obstructive colon carcinoma (from here referred to as 'control resection'). These controls reflect daily practice. Tissue blocks were obtained at a distance of $\geq 10 \mathrm{~cm}$ from the tumour and histologically confirmed as 
normal by haematoxylin and eosin staining. The second control group contained autopsy material of ileum and colon, achieved from patients without bowel motility problems and histologically confirmed as normal (from here referred to as 'control autopsy'). This material was specifically collected to allow for more extensive comparisons of the different locations and orientation of normal tissue. Archived FFPE segments of small bowel and colon from several (secondary) motility disorders were obtained from patients who underwent surgical resection of a part of the bowel: slow transit constipation (STC) and spinal cord injury (SCI; including spina bifida). Also sections from autopsy patients were obtained: amyloidosis and systemic sclerosis (SSc).

The study was approved by the local Ethics Committee (reference number 2014-1256). Samples were obtained in accordance with the Code of Conduct of the Federation of Medical Scientific Societies in the Netherlands. ${ }^{[10]}$

\section{Immunohistochemistry}

FFPE full-thickness tissue blocks were cut in $4 \mu \mathrm{m}$ sections for immunohistochemistry. Most cross-sections were transversely oriented, but also longitudinal and oblique crosssections were included. Sections were deparaffinised, rehydrated in xylene and ethanol series, and rinsed in tap water. CD117 (c-kit) immunohistochemical staining was done by an automated LabVision Autostainer 480 (Klinipath, Duiven, The Netherlands). No antigen retrieval was performed. Subsequently, endogenous peroxidase was blocked with 3\% hydrogen peroxide in methanol for 10 minutes. Sections were incubated with the primary antibody (CD117, clone YR145, Immunologic, Duiven, The Netherlands, dilution 1:200) for 60 minutes. After that, Powervision poly-HRP anti Ms/Rb/Rt was incubated for 30 minutes, followed by staining with PowerDAB for 7 minutes and counterstaining with haematoxylin for one minute. All incubations were performed at room temperature. Tissue blocks containing appendix and gastrointestinal stromal tumour tissue were used as positive controls.

Subsequently, the network of ICCs around the circumference of the myenteric plexus was assessed by light microscopy. The percentage of the circumference which is covered by CD117 positive cells was estimated from $0-100 \%$ in $10 \%$ increments, under $100 \mathrm{x}$ magnification. Thus a percentage of $0 \%$ represented no positive cells around the ganglions (Figure 2.1A). Ganglions in which $10 \%$ of the circumference was covered by CD117 positive cells were scored as $10 \%$, ganglions in which $20 \%$ of the circumference was covered by CD117 positive cells were scored as 20\%, etcetera (Figure 2.1). In sections estimated as $100 \%$, the ganglions were completely surrounded by CD117 positive cells (Figure 2.1D). Staining of mast cells was used as internal positive control.

To study interobserver reliability, two independent observers blinded to the clinical information estimated the CD117 positive network. Mean scores of both observers were used for analysis. For intraobserver comparison, one observer repeated the analyses on 79 samples after $>6$ months period. 

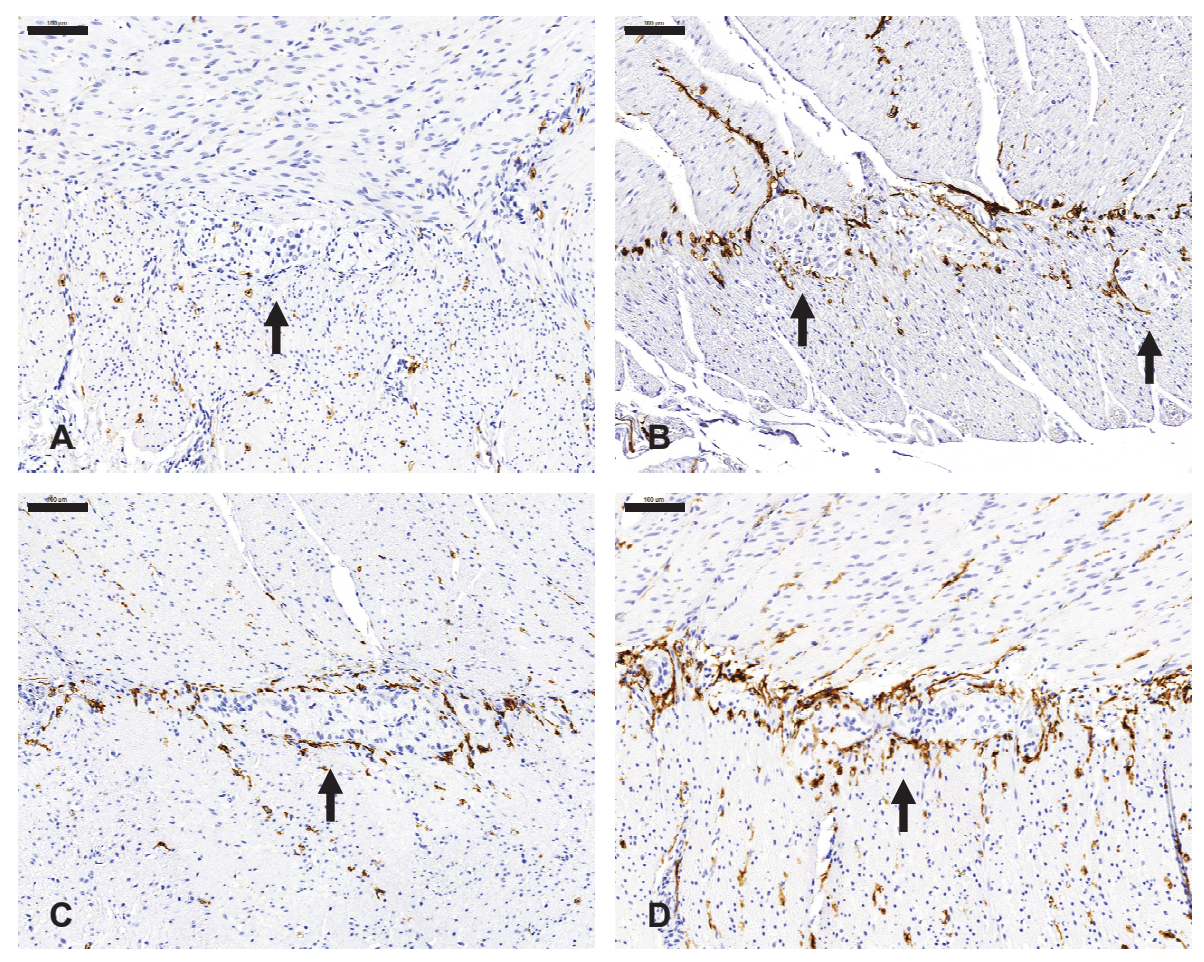

Figure 2.I Estimation of the percentage CDII7 positive cells (ICCs) surrounding the myenteric plexus.

Arrows indicate the ganglions of the myenteric plexus. A) No ICCs are shown around the circumference of the ganglion (0\%). B-D) The ganglions are surrounded by ICCs for 30\% (B), $80 \%$ (C) and $100 \%$ (D) of the circumference, respectively. Pictures demonstrate sections of SCI colon (A), control autopsy ileum (B), control resection ileum (C), and SCI ileum (D). Scalebars $100 \mu \mathrm{m}$. ICC, interstitial cell of Cajal, SCI, spinal cord injury.

\section{Statistical analysis}

The data are presented as means \pm standard deviation (SD). Intraobserver and interobserver agreement and reliability were analysed by the Bland-Altman method and intraclass correlation coefficients based on a two-way mixed model with measures of consistency. ${ }^{[11,12]}$ An intraclass correlation coefficient of 1 indicates a perfect reliability (with no measurement error), whereas 0 represents just reliability based on chance (no reliability). The reliability coefficients were interpreted as follows: $0-0.20$ slight; 0.21 0.4 fair; 0.41-0.60 moderate; 0.61-0.8 substantial, and 0.81-1.00 almost perfect. ${ }^{[13]}$

Comparisons of independent groups were performed using the Mann-Whitney Test, paired samples were tested by the Wilcoxon Signed Ranks Test. A p-value of 0.05 was considered significant. Data were analysed by the IBM SPSS Statistics 20 Software (SPSS Inc., Chicago, IL, USA) and GraphPad Prism version 5.00 for Windows 
(GraphPad Software, San Diego California USA).

\section{Results}

The patient characteristics are given in Table 2.1. The agreement in interobserver and intraobserver analyses is illustrated by Bland-Altman plots (Figure 2.2). In the interobserver analyses, the mean differences were close to zero $(<3 \%)$, indicating that there was no marked systematic error between the observers (Figure 2.2A, B). Twice the standard deviation, which represents the error range of the estimated scores, was $28 \%$ and $18 \%$ in the interobserver analyses of small bowel and colon, respectively. For the interobserver reliability, the scores of small bowel $(n=87)$ and colon $(n=159)$ were used in general, thus without distinguishing between the groups. The interobserver reliability was almost perfect for small bowel (intraclass correlation coefficient 0.847 ; $95 \%$ confidence interval (CI) (0.774-0.897)) and substantial for colon $(0.683 ; 95 \% \mathrm{CI}$ (0.591-0.758)).

One observer repeated the assessment of 79 cases (control resection and SCI cases). The mean difference was 2.2\% and twice the standard deviation was 23\% (Figure 2.2C). The intraobserver reliability was almost perfect (intraclass correlation coefficient 0.918; 95\% CI (0.874-0.947)).

Table 2.I Patient characteristics of the groups included in the study.

\begin{tabular}{|c|c|c|c|c|c|c|}
\hline \multirow[b]{2}{*}{ Group } & \multicolumn{3}{|c|}{ Small bowel } & \multicolumn{3}{|c|}{ Colon } \\
\hline & $\mathbf{n}$ & Male, n (\%) & Age $($ mean \pm SD), year & $\mathbf{n}$ & Male, n (\%) & Age $($ mean $\pm S D)$, year \\
\hline Control resection & 7 & $4(57.1)$ & $72.0 \pm 14.4$ & 16 & $5(31.2)$ & $61.8 \pm 12.6$ \\
\hline Control autopsy & 20 & $11(55.0)$ & $63.2 \pm 16.1$ & 22 & $13(59.1)$ & $63.7 \pm 14.2$ \\
\hline STC & 7 & $1(14.3)$ & $54.7 \pm 12.4$ & 11 & $4(36.4)$ & $56.7 \pm 18.3$ \\
\hline SCI & 8 & $4(50.0)$ & $53.0 \pm 18.8$ & 46 & $21(45.7)$ & $47.8 \pm 19.7$ \\
\hline Amyloidosis & 9 & $7(77.8)$ & $68.3 \pm 17.7$ & 11 & $10(90.9)$ & $66.4 \pm 12.3$ \\
\hline SSc & 16 & $5(31.2)$ & $60.0 \pm 12.3$ & 15 & $5(33.3)$ & $58.7 \pm 12.5$ \\
\hline
\end{tabular}

$S D$, standard deviation, $S C I$, spinal cord injury, $S S c$, systemic sclerosis, $S T C$, slow transit constipation. 

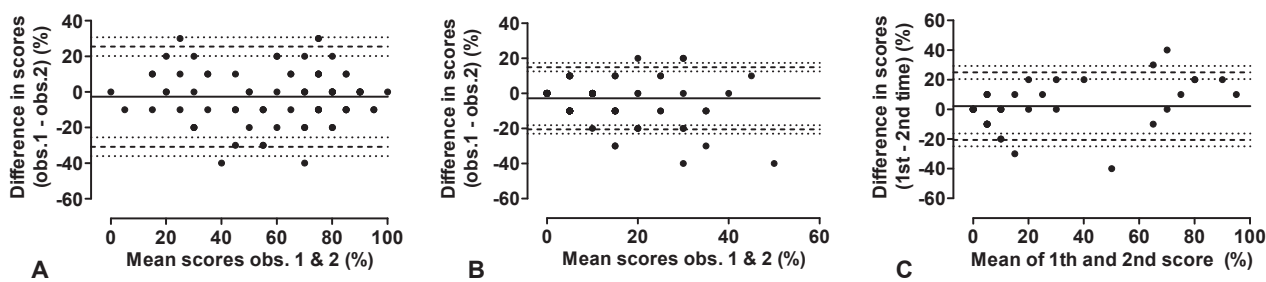

Figure 2.2 Bland-Altman plots of the inter- and intraobserver difference in ICC estimation. Paired differences between estimated ICC scores examined by two observers in the small bowel (A) and colon (B). C) Intraobserver differences between estimated ICC scores. Solid lines represent mean; dashed lines the $95 \%$ limits of agreement, each with $95 \%$ confidence interval (dotted lines). ICC, interstitial cell of Cajal.
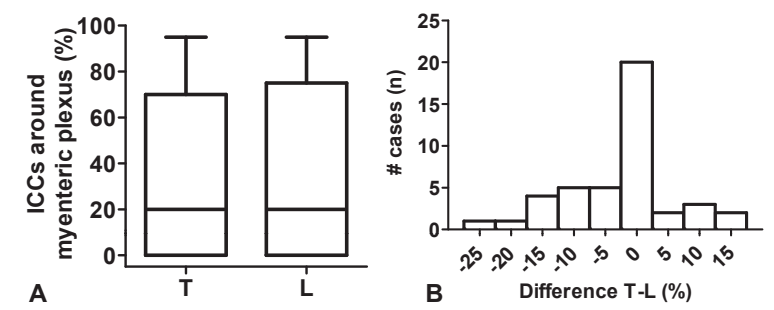

Figure 2.3 Pairwise comparison of myenteric ICC scores in transverse and longitudinal cross-sections $(n=43)$.

A) Distributions of ICC estimation scores in both cross-sections were similar. B) illustrates the difference between scores of transverse and longitudinal cross-sections. Most cases showed no difference $(\mathrm{T}-\mathrm{L}=0 \%)$. $I C C$, interstitial cell of Cajal, $L$, longitudinal $T$, transverse cross-section.

\section{Orientation of the sections}

In 43 cases both transverse and longitudinal cross-sections were pairwise compared (Figure 2.3). No differences were found between transverse (mean $31.6 \pm 33.6 \%$ ) and longitudinal (mean $34.2 \pm 34.4 \%$ ) cross-sections, $\mathrm{p}=0.072$. Hence, for these cases the transverse cross-sections were chosen for further analysis. 


\section{Location of the examined sections}

Sections of patients $(n=39)$ with both small bowel and colon tissue were pairwise compared. Scores given in small bowel tissue were in all cases higher than in colon (mean $60.5 \pm 25.6 \%$ respectively $6.2 \pm 8.2 \%$ ).

Control autopsy samples were systematically collected from different locations in the colon: ascending, transverse and descending colon (Figure 2.4). Scores of ascending colon sections showed no difference with transverse colon scores $(\mathrm{n}=7, \mathrm{p}=$ 0.258). Between ascending and descending colon no differences were found $(\mathrm{n}=5, \mathrm{p}=$ $0.102)$ and no differences between transverse and descending colon as well $(\mathrm{n}=7, \mathrm{p}=$ 0.258). Therefore, the mean CD117 scores of different colonic regions were calculated and used in the following analyses.

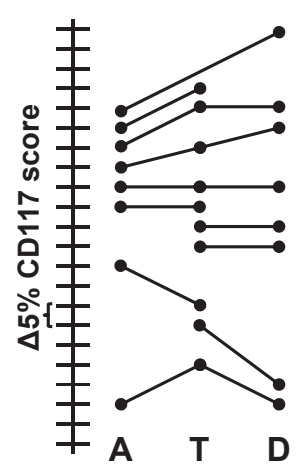

Figure 2.4 Comparison of different regions of control autopsy tissues ( $\mathrm{n}=\mathrm{II}$ ).

No clear differences between ascending $(A)$, transverse $(T)$ and descending $(D)$ colon were found. Each line represents the positive or negative difference in CD117 score within one case. ICC, interstitial cell of Cajal.

\section{Comparison of different groups}

First, results of the estimation of the CD117 positive myenteric ICC network of both control groups were compared. Figure 2.5 shows that no differences were found between the control resection $(\mathrm{n}=7$, mean $75.7 \pm 11.0 \%)$ and control autopsy $(\mathrm{n}=20$, mean $60.5 \pm 29.2 \%)$ groups in the small bowel $(\mathrm{p}=0.420)$. In the colon, higher scores were given in the control resection group $(\mathrm{n}=16$, mean $12.5 \pm 8.0 \%)$ compared with the control autopsy group $(\mathrm{n}=22$, mean $9.0 \pm 13.0 \%)$, although not significant $(\mathrm{p}=0.070)$. 
Second, disease groups consisting of resection tissue were compared with the control resection group (Figure 2.5A). In the small bowel, both the STC group ( $\mathrm{n}=$ 7 , mean $72.1 \pm 23.1 \%)$ and the SCI group $(n=8$, mean $61.3 \pm 24.8 \%)$ were not significantly different from the control group (respectively $\mathrm{p}=0.949$ and $\mathrm{p}=0.245$ ). In the colon, the STC group $(\mathrm{n}=11,16.4 \pm 15.0 \%)$ showed no differences compared with the control group $(\mathrm{p}=0.707)$, but significant lower scores were found in the SCI group $(\mathrm{n}=46$, mean $5.4 \pm 5.7 \%)(\mathrm{p}=0.001)$.

Third, disease groups containing autopsy tissue were compared with the control autopsy group (Figure 2.5B). In the small bowel, no differences were found between the control group and respectively the amyloidosis $(\mathrm{n}=9$, mean $49.4 \pm 18.1 \%)$ and SSc groups $(\mathrm{n}=16$, mean $53.1 \pm 24.6 \%)(\mathrm{p}=0.228$ and $\mathrm{p}=0.306)$. Also in the colon no differences were found in the amyloidosis $(\mathrm{n}=11$, mean $5.5 \pm 9.6 \%)$ and SSc $(\mathrm{n}=15$, mean $10.0 \pm 14.6 \%)$ groups, compared with the control group $(\mathrm{p}=0.365$ respectively $\mathrm{p}=0.843)$.
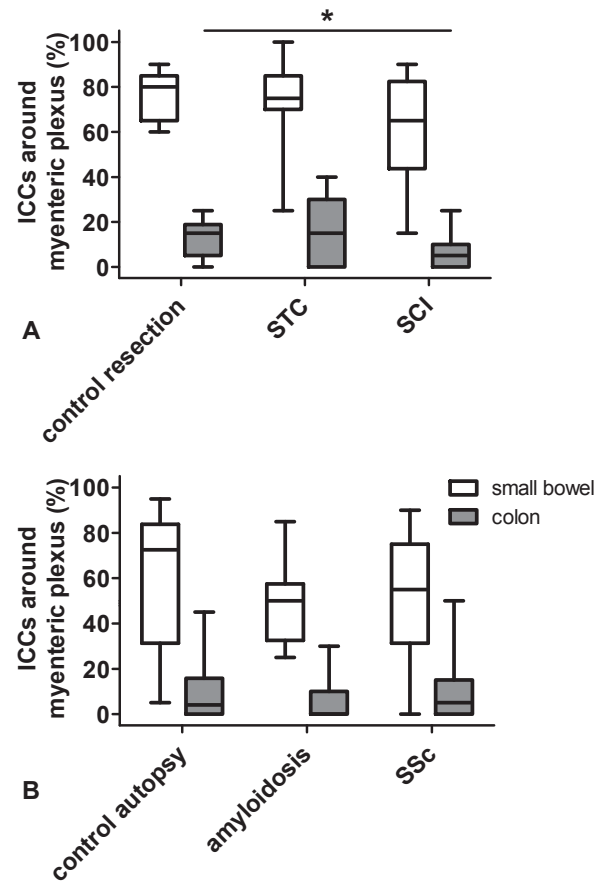

Figure 2.5 Patients with gastrointestinal motility disorders were compared with controls to perform a preliminary evaluation of the estimation method.

Significant less ICCs were found in the colon of SCI patients $\left({ }^{*} \mathrm{p}=0.001\right)$. ICC, interstitial cell of Cajal, $S C I$, spinal cord injury, $S S c$, systemic sclerosis, $S T C$, slow transit constipation. 


\section{Discussion}

The presented estimation method for the ICC network around the circumference of the myenteric plexus shows generally good agreement and reliability. Agreement represents the closeness of two measurements on the same subject, while reliability indicates the capability of a method to distinguish between different subjects despite measurement error. Cases with major inter- or intraobserver variation often contained several tissue samples with different ICC distribution on one slide. Observers could have assessed other tissue areas, explaining the large variation. Therefore, the evaluation of multiple sections per patient is recommended for thorough assessment.

Our estimation method provides a semiquantitative, simple and nearly objective evaluation technique of the myenteric ICC network. Hence, it can easily be implemented in clinical practice. In the small bowel (ileum), mean estimated ICC percentages around the myenteric plexus in control tissues were $75.7 \%$ and $60.5 \%$ for resection and autopsy material, respectively. Bland-Altman analysis showed an error range between the two observers of $30 \%$, which means that only differences of more than $30 \%$ can be distinguished from control tissue and might be diagnosed as disease. Hence, myenteric ICC percentages lower than $50 \%$ in resection material and below $30 \%$ in autopsy material can be detected with our method. The international guidelines suggested reporting a reduction in ICC number of more than $50 \%$ as abnormal. ${ }^{[1]}$ Applying the guidelines to our normal values, myenteric ICC scores below $40 \%$ in resection material and lower than $30 \%$ in autopsy material should be diagnosed as abnormal. Therefore, our method can be implemented in the clinical practice following the guidelines.

In the colon, differences larger than $20 \%$ could be differentiated as abnormal, because the rater variability between the two observers was $20 \%$. Our control samples showed mean estimated ICCs around the myenteric plexus of $12.5 \%$ in resection and $9.0 \%$ in autopsy material suggesting that only the complete absence of ICCs should be diagnosed as abnormal. However, to provide proper normal values, larger cohorts of different origins need to be evaluated. Moreover, our normal values were obtained from adult patients and may not be applicable for children.

Our estimation method was able to distinguish between normal and diseased intestinal tissue, as shown by the results of the preliminary evaluation of control groups and several disease groups. Significant less ICCs surrounding the myenteric plexus of the colon in SCI patients were found, compared with control samples, as illustrated in Figure 2.1A. However, the difference between the mean estimated ICC percentages of these groups was $7 \%$ and could be a result of rater variability, because the confidence interval of rater agreement was $+/-20 \%$ for the colon samples. In addition, since for a thorough examination of dysmotility all components of the neuromuscular system should be evaluated, no firm conclusions can be drawn from this observation. 
In the current study, control material of resections was used to represent the clinical setting. Furthermore, we used autopsy control material to study additional clinically relevant questions. Firstly, transversally and longitudinally oriented crosssections were compared and no significant differences between scores were found. Consequently, assessment of the myenteric ICCs can be performed on tissues from either transverse or longitudinal orientation in daily practice. Secondly, different locations of the bowel were compared. The myenteric ICC network was always more prominent in the small bowel than in the colon. On the other hand, our findings suggest no regional differences in the colon, in contrast to results from a previous study. ${ }^{[14]}$ However, we compared only a small group consisting of autopsy material. Since it has been suggested that it is very important to compare normal and pathologic tissues from the same bowel region ${ }^{[15-17]}$, comparison of material from several colonic locations have to be repeated on resection material instead of autopsy material with the presented method.

In conclusion, we proposed an estimation method for evaluation of the myenteric ICC network in the bowel with acceptable agreement and reliability. In the small bowel, myenteric ganglions surrounded by less than $40 \%$ of ICCs may be safely reported as abnormal (in resection material), whereas in the colon only tissues with completely absent myenteric ICCs might be diagnosed as abnormal. This method has potential to be implemented in routine diagnostics of intestinal neuromuscular disorders, corresponding to the international guidelines. 


\section{References}

1. Knowles CH, De Giorgio R, Kapur RP, et al. Gastrointestinal neuromuscular pathology: guidelines for histological techniques and reporting on behalf of the Gastro 2009 International Working Group. Acta Neuropathol, 2009. 118(2): p. 271-301.

2. Komuro T. Atlas of Interstitial Cells of Cajal in the Gastrointestinal Tract. 2012, Dordrecht: Springer Science \& Business Media.

3. Streutker CJ, Huizinga JD, Driman DK, et al. Interstitial cells of Cajal in health and disease. Part I: normal ICC structure and function with associated motility disorders. Histopathology, 2007. 50(2): p. 176-189.

4. Wedel T, Spiegler J, Soellner S, et al. Enteric nerves and interstitial cells of Cajal are altered in patients with slow-transit constipation and megacolon. Gastroenterology, 2002. 123(5): p. 14591467.

5. Wang LM, McNally M, Hyland J, et al. Assessing interstitial cells of Cajal in slow transit constipation using CD117 is a useful diagnostic test. Am J Surg Pathol, 2008. 32(7): p. 980-985.

6. Gao J, Du P, O'Grady G, et al. Numerical metrics for automated quantification of interstitial cell of Cajal network structural properties. J R Soc Interface, 2013. 10(86): 20130421.

7. Huizinga JD, Martz S, Gil V, et al. Two independent networks of interstitial cells of cajal work cooperatively with the enteric nervous system to create colonic motor patterns. Front Neurosci, 2011. 5: p. 93.

8. Blair PJ, Rhee PL, Sanders KM, et al. The significance of interstitial cells in neurogastroenterology. J Neurogastroenterol Motil, 2014. 20(3): p. 294-317.

9. Al-Shboul OA. The importance of interstitial cells of cajal in the gastrointestinal tract. Saudi J Gastroenterol, 2013. 19(1): p. 3-15.

10. Code of Conduct of the Dutch federation of Medical Scientific Societies. [Internet]. Available from: http://www.federa.org. Accessed 8 Dec 2014.

11. Shrout PE, Fleiss JL. Intraclass correlations: uses in assessing rater reliability. Psychol Bull, 1979. 86(2): p. 420-428.

12. Bland JM, Altman DG. Statistical Methods for Assessing Agreement between Two Methods of Clinical Measurement. Lancet, 1986. 1(8476): p. 307-310.

13. Landis JR, Koch GG. The measurement of observer agreement for categorical data. Biometrics, 1977. 33(1): p. 159-174.

14. Hagger R, Gharaie S, Finlayson C, et al. Regional and transmural density of interstitial cells of Cajal in human colon and rectum. Am J Physiol, 1998. 275(6 Pt 1): p. G1309-1316.

15. Horisawa M, Watanabe Y, Torihashi S. Distribution of c-Kit immunopositive cells in normal human colon and in Hirschsprung's disease. J Pediatr Surg, 1998. 33(8): p. 1209-1214.

16. Torihashi S, Horisawa M, Watanabe Y. c-Kit immunoreactive interstitial cells in the human gastrointestinal tract. J Auton Nerv Syst, 1999. 75(1): p. 38-50.

17. Vanderwinden JM, Rumessen JJ. Interstitial cells of Cajal in human gut and gastrointestinal disease. Microsc Res Tech, 1999. 47(5): p. 344-360. 
Practical estimation of myenteric ICCs 


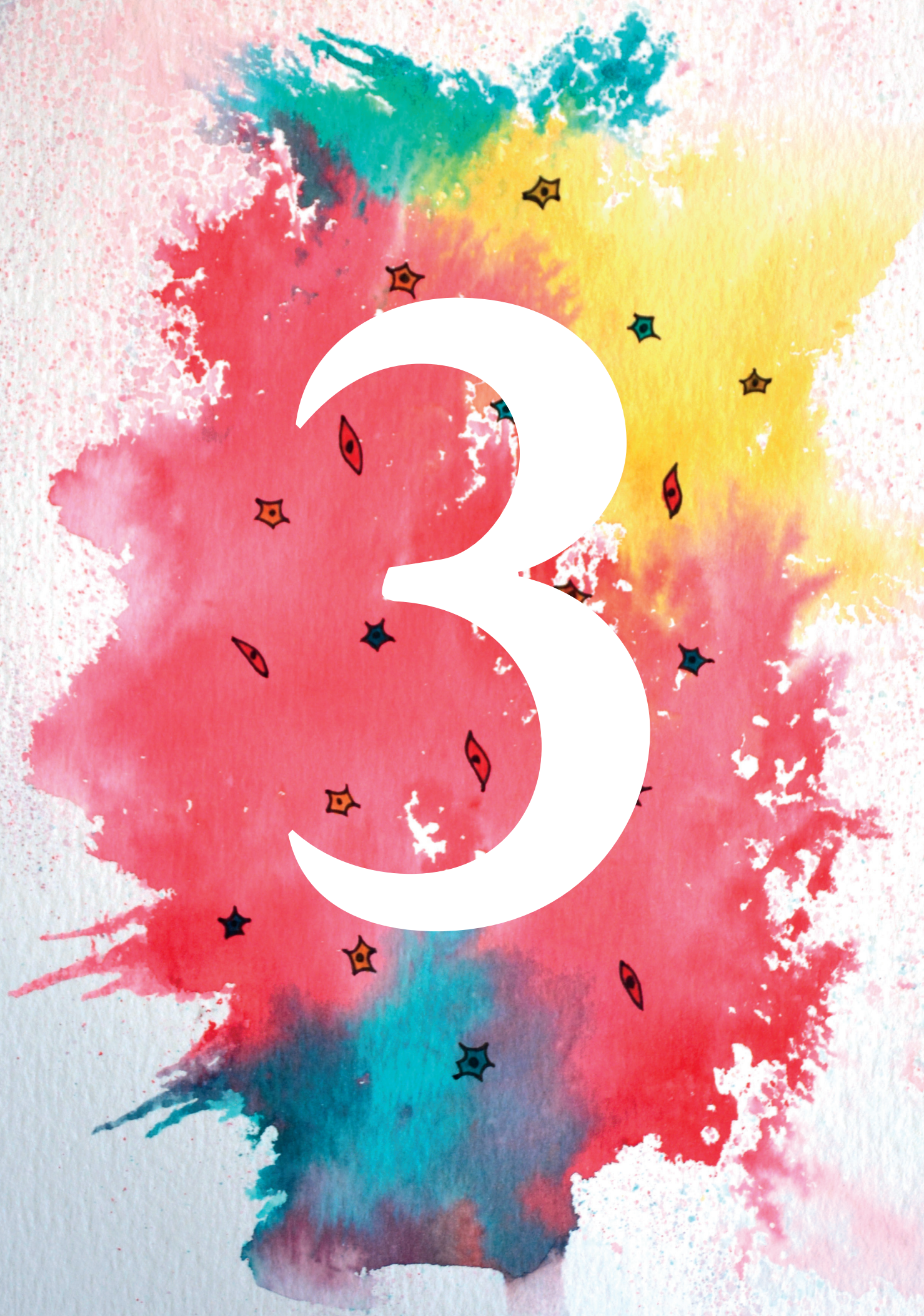




\section{Chapter 3}

\section{Semiquantitative methods}

Adapted from Supplemental Figures of:

The enteric nervous system and the musculature of the colon are altered in patients with spina bifida and spinal cord injury.

M den Braber-Ymker ${ }^{1}$, M Lammens ${ }^{1,2,3}$, M.J.A.M. van Putten ${ }^{4,5}$, I.D. Nagtegaal ${ }^{1}$
${ }^{1}$ Department of Pathology, Radboud University Medical Center, Nijmegen, The Netherlands
${ }^{2}$ Department of Pathology, Antwerp University Hospital, University of Antwerp, Edegem, Belgium
${ }^{3}$ MIPRO, University of Antwerp, Antwerp, Belgium
University of Twente, Enschede, The Netherlands
${ }^{4}$ Department of Clinical Neurophysioloy, MIRA, Instite for Biomedical Technology and Technil Medicine,
${ }^{5}$ Department of Neurology and Clinical Neurophysiology, Medisch Spectrum Twente, Enschede, The Netherlands

Virchows Arch, 2017. 470(2): p. 175-184 
We introduced a semiquantitative scoring method for systematic analysis of the ENS and smooth muscle layers on immunohistochemically stained tissue sections. These semiquantitative scoring methods were used in chapters 4 to 6 .

\section{Antibodies and staining methods}

Sections of $4 \mu \mathrm{m}$ were cut from formalin-fixed paraffin-embedded full-thickness tissue blocks. Antibodies, suppliers and dilutions are listed in Table 3.1.

For $\mathrm{HuC} / \mathrm{D}$ staining, antigen retrieval was performed in sodium citrate $(\mathrm{pH}$ 6) at $100{ }^{\circ} \mathrm{C}$ for $30 \mathrm{~min}$. Subsequently, endogenous peroxidase was blocked with $3 \%$ hydrogen peroxide in PBS for $20 \mathrm{~min}$. Sections were then rinsed in PBS and incubated with primary antibody anti-HuC/D at $4{ }^{\circ} \mathrm{C}$ overnight. After washing in PBS, sections were incubated for 30 min with a secondary antibody (Powervision poly-HRP anti Ms/ $\mathrm{Rb} / \mathrm{Rt}$ IgG, Immunologic, Duiven, The Netherlands) at room temperature. Sections were finally rinsed in PBS and immunoreactivity was developed with PowerDAB (Immunologic) for $7 \mathrm{~min}$ at room temperature. Subsequently, sections were rinsed in tap water, counterstained with haematoxylin, rinsed in tap water, dehydrated in $100 \%$ ethanol and xylene, and mounted with Permount.

The other immunohistochemical staining reactions were performed in an automated LabVision Autostainer 480 (Klinipath, Duiven, The Netherlands). First, the method used for antigen retrieval depended on the antibody (Table 3.1). Subsequently, endogenous peroxidase was blocked with $3 \%$ hydrogen peroxide in methanol for $10 \mathrm{~min}$. Sections were incubated with primary antibody for $60 \mathrm{~min}$. Subsequently, the sections were incubated with Powervision poly-HRP anti Ms/Rb/Rt for $30 \mathrm{~min}$, followed by staining with PowerDAB for $7 \mathrm{~min}$ and counterstaining with haematoxylin for $1 \mathrm{~min}$. All incubations were performed at room temperature.

Tissue blocks containing different tissue types were used as controls, with known staining patterns for both positive and negative stained tissues.

Table 3.I Primary antibodies used for immunohistochemistry.

\begin{tabular}{|c|c|c|c|c|}
\hline Antibody & Clone & Manufacturer & Dilution & Antigen retrieval \\
\hline Calretinin & $5 \mathrm{~A} 5$ & Novocastra & $1: 25$ & EDTA pH $910 \mathrm{~min}$ at $96^{\circ} \mathrm{C}$ \\
\hline $\mathrm{HuC} / \mathrm{D}$ & $16 \mathrm{~A} 11$ & Molecular Probes & $1: 600$ & $\begin{array}{l}\text { Sodium citrate } 10 \mathrm{mM}(\mathrm{pH} \text { 6.0) } \\
30 \text { min at } 100^{\circ} \mathrm{C}\end{array}$ \\
\hline$S 100$ & polyclonal & DAKO & $1: 10000$ & EDTA pH $910 \mathrm{~min}$ at $96^{\circ} \mathrm{C}$ \\
\hline CD117 & YR145 & Immunologic & $1: 200$ & None \\
\hline $\begin{array}{l}\alpha \text {-smooth muscle } \\
\text { actin }(\alpha \text {-SMA })\end{array}$ & $1 \mathrm{~A} 4$ & Sigma & $1: 7500$ & None \\
\hline Desmin & 33 & Biogenex & $1: 100$ & Citrate $\mathrm{pH} 6.730 \mathrm{~min}$ at $100^{\circ} \mathrm{C}$ \\
\hline
\end{tabular}




\section{Semiquantitative analysis}

\section{Neuronal density}

The presence of neurons in ganglia of the submucosal and myenteric plexus was analysed on $\mathrm{HuC} / \mathrm{D}$ - and calretinin-stained sections. $\mathrm{HuC} / \mathrm{D}$ is a pan-neuronal marker for enteric nerve cells, visualising the perikarya but not neuronal processes. ${ }^{[1,2]}$ Calretinin is present in perikarya and nerve processes of a subset of enteric neurons, including intrinsic primary afferent neurons. ${ }^{[3,4]}$ Thus, $\mathrm{HuC} / \mathrm{D}$ stains the whole neuron population, while calretinin stains only a subset (Figure 3.1).

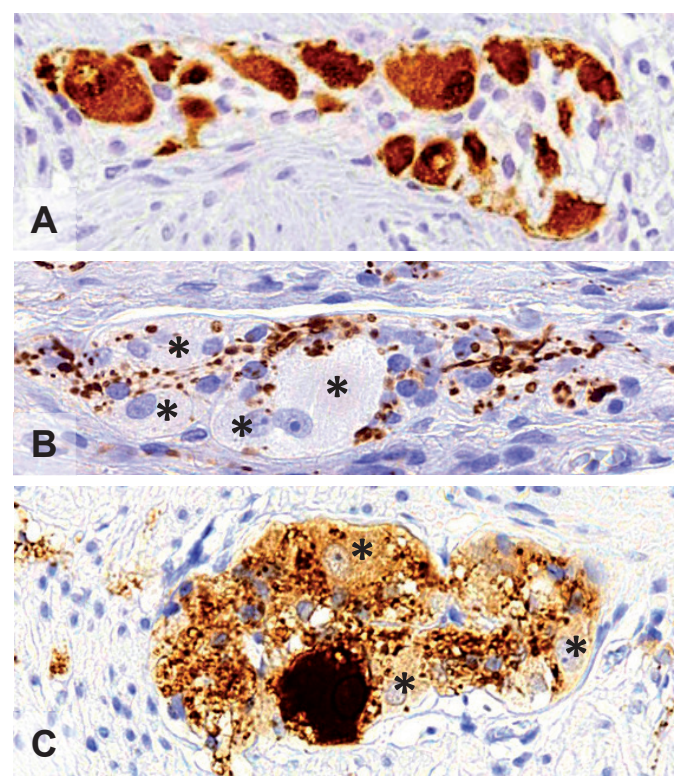

Figure 3.I. Comparison of HuC/D and calretinin.

A) The perikarya of all neurons in the ganglion are stained by $\mathrm{HuC} / \mathrm{D}$. B and C) A subset of neurons is stained by calretinin (large brown cell in C). Neurons negative for calretinin are indicated with asterisks, nerve processes are positively stained by calretinin.

The number of neurons in relation to the present plexus was estimated in $\mathrm{HuC} / \mathrm{D}$ sections as follows: Firstly, the distribution of the neuronal network was evaluated on S100-stained sections to determine the location and size of the plexus. Subsequently, the number of neurons per ganglion was estimated in the $\mathrm{HuC} / \mathrm{D}$ staining in relation to the distribution of the neuronal network and scored as 0 (no neurons), 1 (low neuronal density), and 2 (high neuronal density); Figure 3.2. The calretinin-stained sections were comparably rated: 0 (no neurons), 1 (on average less than one neuron per ganglion), and 2 (minimal one neuron per ganglion); Figure 3.3. 

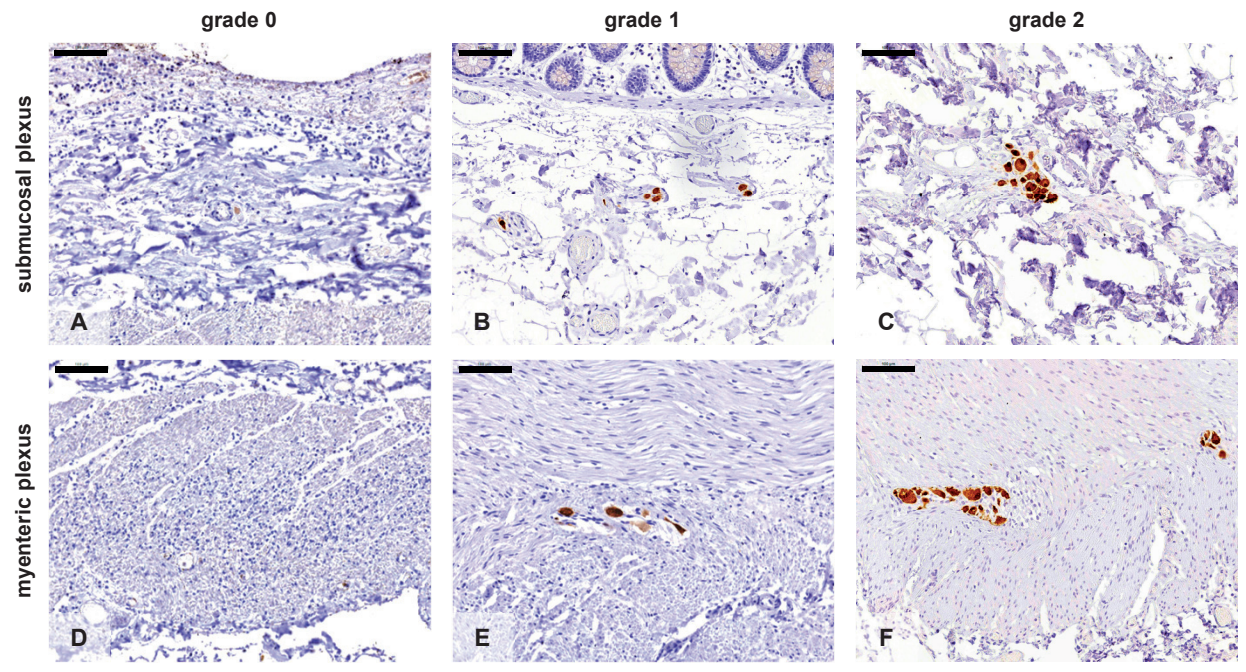

Figure 3.2 Semiquantitative scoring of HuC/D stained sections.

The number of neurons in relation to the present plexus was estimated in $\mathrm{HuC} / \mathrm{D}$ sections as follows: Firstly, the distribution of the neuronal network was evaluated on S100 stained sections. Subsequently, the number of neurons per ganglion was estimated in the $\mathrm{HuC} / \mathrm{D}$ staining in relation to this neuronal network and scored as no neurons (grade 0), low neuronal density (grade 1), and high neuronal density (grade 2). Scalebars $100 \mu \mathrm{m}$.

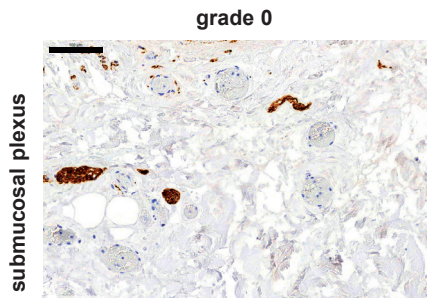

A

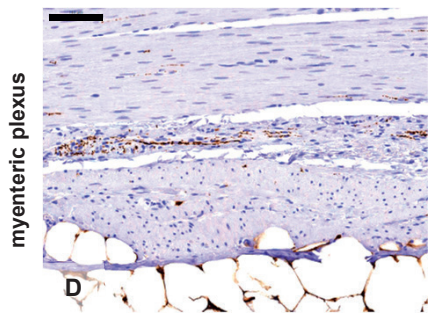

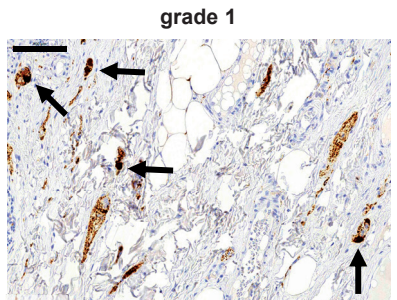

B

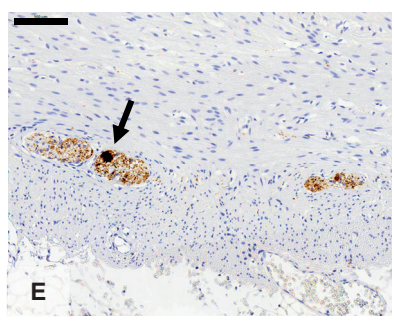

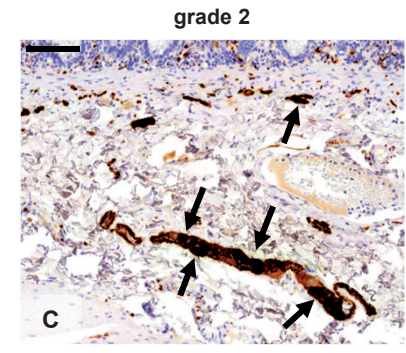

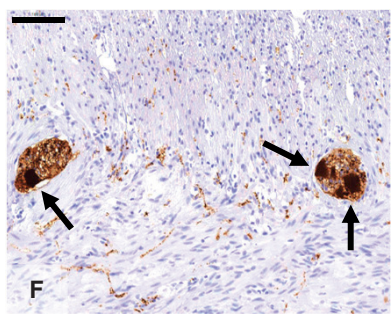

Figure 3.3 Semiquantitative scoring of calretinin stained sections.

The number of neurons was estimated in relation to the present plexus: no neurons (grade 0), on average less than one neuron per neuronal structure (grade 1), minimal one neuron per neuronal structure (grade 2). Neurons are indicated with arrows. Scalebars $100 \mu \mathrm{m}$. 


\section{Glial cell density}

S100 is a specific marker for nerve fibres as well as enteric glial cells (nucleus and cytoplasm). ${ }^{[5]}$ S100 staining was used to assess the distribution of nerve fibres and glial cells in the submucosa, the myenteric plexus, and both muscle layers of the muscularis propria. The degree of distribution was scored as follows: 0 (no/low density) and 1 (high density of positive fibres); Figure 3.4.

\section{Smooth muscle layers}

$\alpha$-Smooth muscle actin ( $\alpha$-SMA) and desmin staining were used to assess the muscular layers. $\alpha$-SMA plays a role in contraction of the smooth muscle cells and is the most commonly used marker for smooth muscle cells in the vascular walls and the muscularis layers in the intestines (cytoplasm) ${ }^{[6]}$ The circular muscle layer of the ileum physiologically shows no or low immunoreactivity for $\alpha$-SMA and can therefore not be assessed for this staining (Figure 3.5A). Desmin in the smooth muscle cells can serve as another marker for smooth muscle cells (cytoplasm). Desmin plays no role in contractility, but is essential for maintaining the orientation of actin and myosin filaments. ${ }^{[7]}$ Staining intensities of $\alpha$-SMA and desmin in the circular and longitudinal muscle layers were classified in two grades: 0 (no/weak) and 1 (strong staining intensity), Figure 3.5. Immunoreactivity within blood vessel walls and muscularis mucosae acted as internal reference for $\alpha$-SMA and desmin, respectively (grade 1). 


\section{Chapter 3}
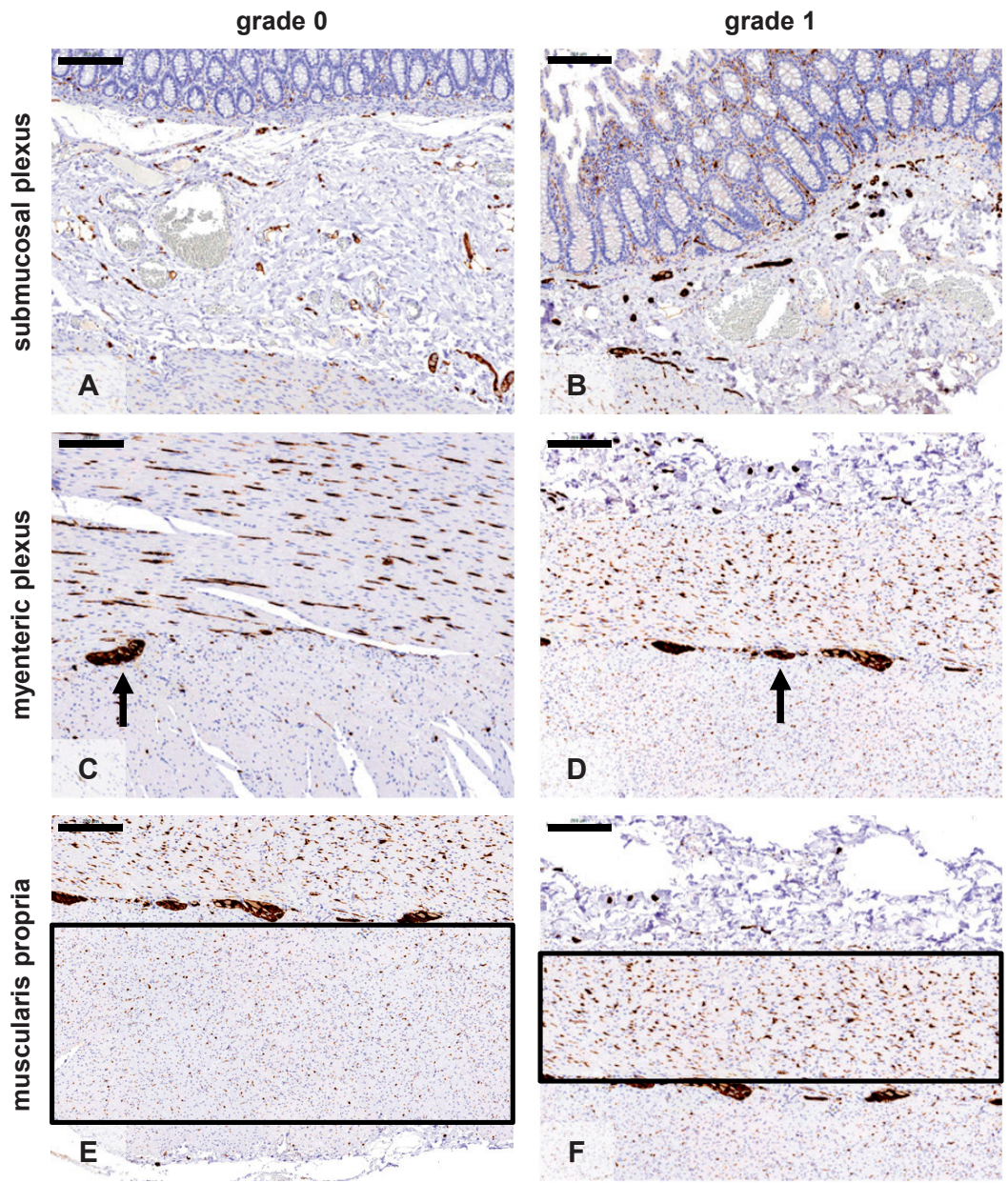

Figure 3.4 Semiquantitative scoring of SI00 stained sections.

The density of nerve fibres was assessed in the submucosal plexus (A, B), the myenteric plexus (arrows) (C, $\mathrm{D}$ ) and the muscularis propria (area within rectangle) (E, F) as follows: no or low density (grade 0 ) and high density of $S 100$ positive fibres (grade 1). Scalebars $200 \mu \mathrm{m}$. 

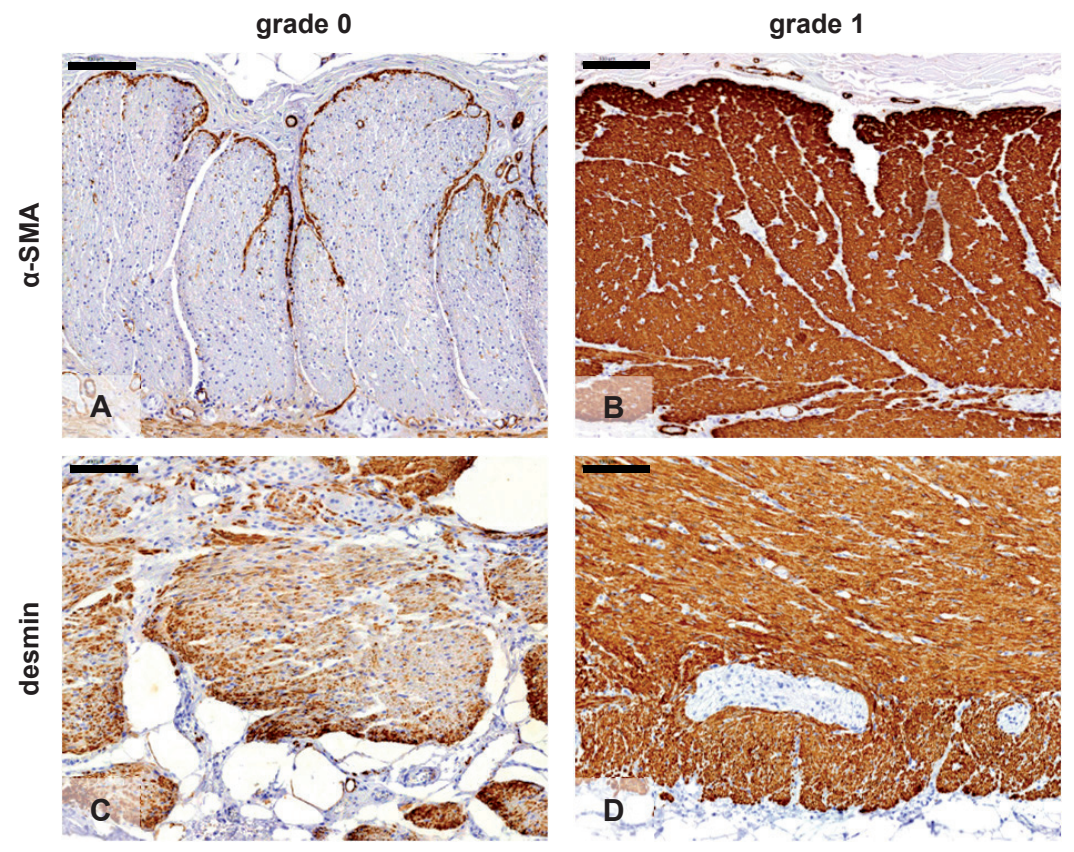

Figure 3.5 Semiquantitative scoring of $\alpha$-Smooth muscle actin (SMA) (A, B) and desmin (C, D) stained sections.

Staining intensities were scored as follows: no or weak staining (grade 0 ) and strong staining intensity (grade 1). Internal references for $\alpha$-SMA and desmin were respectively immunoreactivity within the blood vessel wall and muscularis mucosae (grade 1). Scalebars $100 \mu \mathrm{m}$. 


\section{References}

1. Lin Z, Gao N, Hu HZ, et al. Immunoreactivity of Hu proteins facilitates identification of myenteric neurones in guinea-pig small intestine. Neurogastroenterol Motil, 2002. 14(2): p. 197-204.

2. Phillips RJ, Hargrave SL, Rhodes BS, et al. Quantification of neurons in the myenteric plexus: an evaluation of putative pan-neuronal markers. J Neurosc Methods, 2004. 133(1-2): p. 99-107.

3. Kustermann A, Neuhuber W, Brehmer A. Calretinin and somatostatin immunoreactivities label different human submucosal neuron populations. Anat Rec (Hoboken), 2011. 294(5): p. 858-869.

4. Furness JB. The enteric nervous system and neurogastroenterology. Nat Rev Gastroenterol Hepatol, 2012. 9(5): p. 286-294.

5. Dzienis-Koronkiewicz E, Debek W, Sulkowska M, et al. Suitability of Selected Markers for Identification of Elements of the Intestinal Nervous System (INS). Eur J Pediatr Surg, 2002. 12(06): p. 397-401.

6. Bernardini N, Ippolito C, Segnani C, et al. Histopathology in gastrointestinal neuromuscular diseases: methodological and ontological issues. Adv Anat Pathol, 2013. 20(1): p. 17-31.

7. Paulin D, Li Z. Desmin: a major intermediate filament protein essential for the structural integrity and function of muscle. Exp Cell Res, 2004. 301(1): p. 1-7. 
Semiquantitative methods 


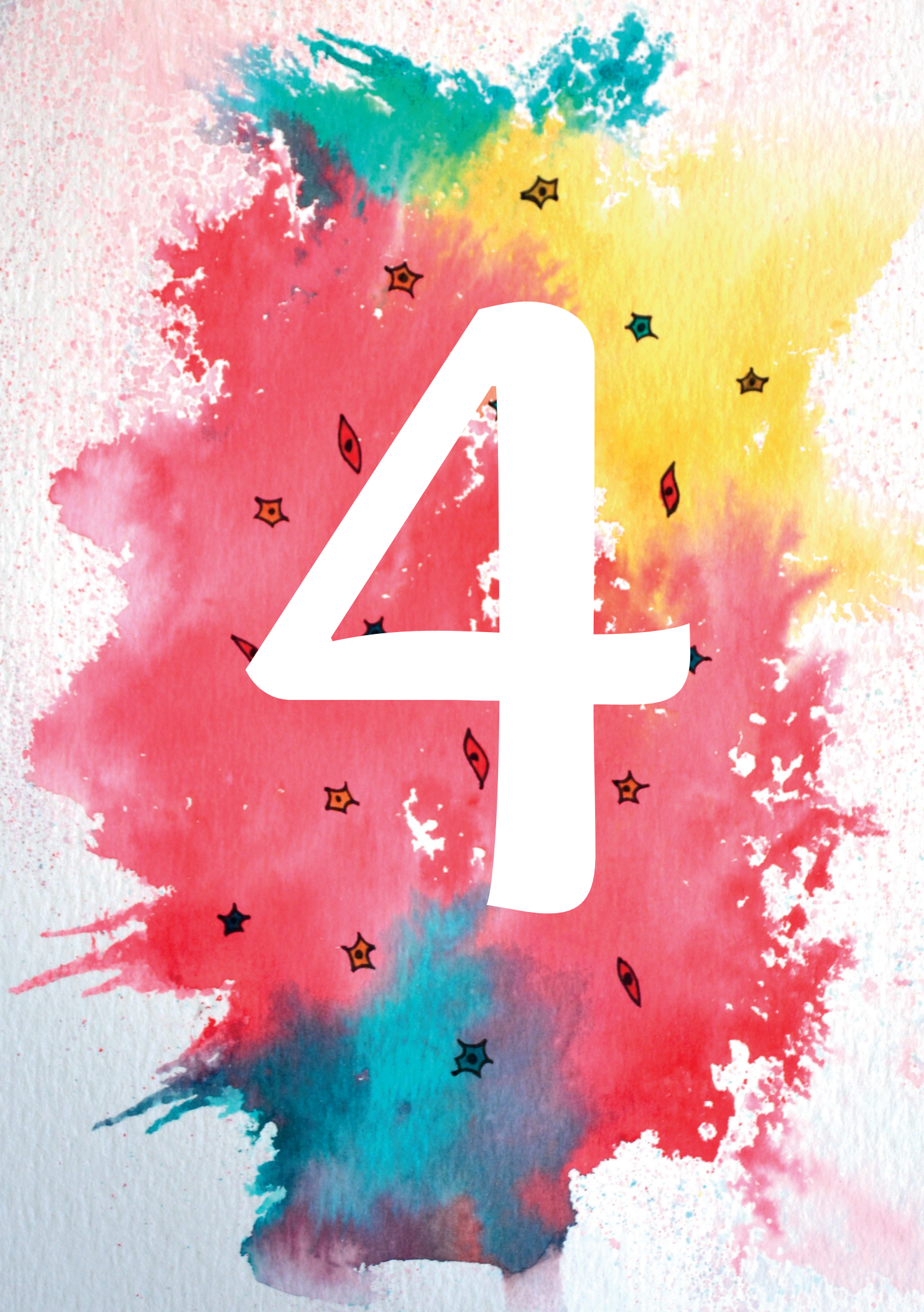




\section{Chapter 4}

\section{The enteric nervous system and the musculature of the colon are altered in patients with spina bifida and spinal cord injury}

M. den Braber-Ymker ${ }^{1}$, M. Lammens ${ }^{1,2,3}$, M.J.A.M. van Putten ${ }^{4,5}$, I.D. Nagtegaal ${ }^{1}$

${ }^{1}$ Department of Pathology, Radboud University Medical Center, Nijmegen, The Netherlands

${ }^{2}$ Department of Pathology, Antwerp University Hospital, University of Antwerp, Edegem, Belgium

${ }^{3}$ MIPRO, University of Antwerp, Antwerp, Belgium

${ }^{4}$ Department of Clinical Neurophysiology, MIRA, Institute for Biomedical Technology and Technical Medicine, University of Twente, Enschede, The Netherlands

${ }^{5}$ Department of Neurology and Clinical Neurophysiology, Medisch Spectrum Twente, Enschede, The Netherlands

Virchows Arch, 2017. 470(2): p. 175-184 


\section{Abstract}

Neurogenic bowel dysfunction occurs in a large percentage of adult patients with spina bifida (SB) and spinal cord injury (SCI), significantly affecting their quality of life. Although bowel motility is autonomously regulated by the enteric nervous system (ENS), disruption of the modulation of the ENS by extrinsic innervation as present in many patients with SB and SCI might lead to motility disorders. In order to gain insight in the pathophysiology, we studied histological changes of the neuromuscular structures in the colon of SB and SCI patients.

Archival colon tissue blocks from SB $(n=13)$ and SCI $(n=34)$ patients were collected nationwide in The Netherlands and compared with control samples $(n=16)$. Histological (semiquantitative) evaluation of the ENS, the network of interstitial cells of Cajal (ICC), and the muscularis propria was performed using haematoxylin and eosin, periodic acid Schiff, and elastic von Gieson staining, and immunohistochemistry with antibodies against $\mathrm{HuC} / \mathrm{D}$, calretinin, S100, CD117, $\alpha$-smooth muscle actin, and desmin.

Compared to controls, SB and SCI patients showed neuronal loss and decreased nerve fibre density in the myenteric plexus. Lower nerve fibre density was significantly more often found in patients with severe bowel dysfunction. Other major findings were loss of ICCs around the myenteric plexus and fibrosis in the longitudinal muscle layer.

Altered histology of the ENS may explain abnormal intestinal motility in SB and SCI patients. Furthermore, loss of myenteric nerve fibres (including enteric glial cells) may play a major role in the development of severe motility complaints. 


\section{Introduction}

Spina bifida (SB) and spinal cord injury (SCI) are both disorders of the central nervous system (CNS). Neurogenic bowel dysfunction is very common following SB and SCI, in approximately $40 \%$ and $42-81 \%$ of adult patients, respectively. ${ }^{[1-3]}$ In $39 \%$ of SCI patients, colorectal dysfunction has a variable but sometimes significant impact on social activities or quality of life. ${ }^{[3,4]}$ Main symptoms include loss of bowel control (faecal incontinence), constipation, and lack of bowel movements. ${ }^{[2,5-8]}$ The occurrence of these complications depends at least partly on the type and the level of the lesion and the time since the injury. ${ }^{[1,9]}$

Motility and secretion in the colon are controlled by both intrinsic and extrinsic innervation. The intrinsic enteric nervous system (ENS) has two major parts: the submucosal plexus and the myenteric plexus, which are located between the circular and longitudinal layers of the muscularis propria. The submucosal plexus mainly controls mucosal secretion and blood flow, while the myenteric plexus is primarily involved in coordination of motility patterns. ${ }^{[10,11]}$ One of the local reflexes regulated by the ENS is the peristaltic reflex, which is responsible for normal propulsion of bowel contents. Neurons involved in this reflex include (sensory) intrinsic primary afferent neurons (IPANs), interneurons and motor neurons. Motor neurons give excitatory and inhibitory signals via the interstitial cells of Cajal (ICCs) to smooth muscle cells. ${ }^{[12]}$ Enteric ganglia contain both neurons and glial cells, which play a supportive role to the neurons. Enteric glial cells are increasingly recognised as important for regulatory functions in the gut, such as the control of motility. ${ }^{[13]}$ Although ICC networks are found in different layers of the bowel wall, the ICC network around the circumference of the myenteric plexus may play a major role in peristalsis. ${ }^{[14-16]}$

The ENS functions largely independently from the CNS, although extrinsic input from the sympathetic and parasympathetic nerves modulates the activity of the ENS. ${ }^{[17,18]}$ Parasympathetic fibres facilitate contraction of the colonic musculature and sympathetic fibres inhibit colonic motility. ${ }^{[19]}$ Hence, disruption of the extrinsic nerve fibres as in SB and SCI has a major effect on ENS activity and results in an abnormal motor function. ${ }^{[20]}$ The resulting intestinal dysfunction is called neurogenic bowel. ${ }^{[4]}$ This clinically well-known phenomenon is rarely studied on morphological level. ${ }^{[21]}$

Morphological analysis of motility disorders is difficult, since a wide variety of morphological and functional alterations of the ENS, ICCs and smooth muscle tissue may result in gastrointestinal neuromuscular diseases (GINMDs). Despite the recent introduction of a classification of these alterations by an International Working Group $^{[22]}$, the correlation between clinical manifestations and morphological findings remains challenging due to the lack of systematic studies. Therefore, we performed this nationwide study to evaluate the histopathology of the colon in SB and SCI. This is the first systematic study investigating morphological alterations in these patient 
groups, which might provide additional insight into the underlying pathophysiology of bowel dysfunction in SB and SCI. Since the aetiology of SB and SCI varies, different histological features might be expected in both groups.

The aim of this study was to describe histological alterations of the neuromuscular apparatus in the colon following SB and SCI. Systematic (semiquantitative) assessment involved the main relevant neuromuscular structures of the bowel (ENS, ICCs, smooth muscle). We applied the proposed international guidelines on histological reporting for gastrointestinal neuromuscular pathologies. ${ }^{[22,23]}$

\section{Methods}

\section{Subjects}

Patients were selected using the nationwide network and registry of histopathology and cytopathology in the Netherlands (PALGA database), which registers all pathologic reports since $1991 .^{[24]}$ Subsequently, archived formalin-fixed paraffin-embedded segments of colon were obtained from patients with SB $(n=13)$ or SCI $(n=34)$ who underwent surgical resection of part of the bowel. In seven patients, the site of the removed segment was not indicated; in seven patients, this was the proximal bowel and in 33 patients the distal bowel.

Control segments of colon $(n=16)$ were obtained from patients who underwent right-sided hemicolectomy for non-obstructive colon carcinoma. Control patients showed no evidence for gastrointestinal motility disorders. Tissue blocks were obtained at a distance of $\geq 10 \mathrm{~cm}$ from the tumour, and these were histologically confirmed as normal.

The study was approved by the local Ethics Committee (reference number 2014-1256). Samples were obtained in accordance with the Code of Conduct of the Federation of Medical Scientific Societies in the Netherlands. ${ }^{[25]}$

\section{Tissue preparation}

Sections were cut from formalin-fixed paraffin-embedded full-thickness tissue blocks for conventional histology or immunohistochemistry. In the patient groups, most sections were transversal ( $\mathrm{SB} n=10$; $\mathrm{SCI} n=22)$, but some were longitudinal ( $\mathrm{SB} n=1$; $\mathrm{SCI}$ $\mathrm{n}=5$ ) or tangled (SB $\mathrm{n}=3$; SCI $\mathrm{n}=8$ ). In the control group, only transversely oriented sections were available.

Sections were deparaffinised by standard protocol in xylene, rehydrated in an ethanol series, and rinsed in tap water. 


\section{Histological staining}

Sections of $4 \mu \mathrm{m}$ were used for haematoxylin and eosin (H\&E) and periodic acid Schiff (PAS) staining. Elastic von Gieson (EVG) staining was performed on $6-\mu \mathrm{m}$ sections. Tissues were stained by standard protocols in a Medite TST 30 stainer (Klinipath, Duiven, The Netherlands).

\section{Immunohistochemistry}

Immunohistochemical staining was performed on $4-\mu \mathrm{m}$ sections. Antibodies, suppliers and dilutions are listed in Table 4.1.

For $\mathrm{HuC} / \mathrm{D}$ staining, antigen retrieval was performed in sodium citrate $(\mathrm{pH}$ 6) at $100{ }^{\circ} \mathrm{C}$ for $30 \mathrm{~min}$. Subsequently, endogenous peroxidase was blocked with $3 \%$ hydrogen peroxide in PBS for $20 \mathrm{~min}$. Sections were then rinsed in PBS and incubated with primary antibody anti-HuC/D at $4{ }^{\circ} \mathrm{C}$ overnight. After washing in PBS, sections were incubated for 30 min with a secondary antibody (Powervision poly-HRP anti Ms/ $\mathrm{Rb} / \mathrm{Rt}$ IgG, Immunologic, Duiven, The Netherlands) at room temperature. Sections were finally rinsed in PBS and immunoreactivity was developed with PowerDAB (Immunologic) for $7 \mathrm{~min}$ at room temperature. Subsequently, sections were rinsed in tap water, counterstained with haematoxylin, rinsed in tap water, dehydrated in $100 \%$ ethanol and xylene, and mounted with Permount.

The other immunohistochemical staining reactions were performed in an automated LabVision Autostainer 480 (Klinipath, Duiven, The Netherlands). First, the method used for antigen retrieval depended on the antibody (Table 4.1). Subsequently, endogenous peroxidase was blocked with 3\% hydrogen peroxide in methanol for $10 \mathrm{~min}$. Sections were incubated with primary antibody for $60 \mathrm{~min}$. Subsequently, the sections were incubated with Powervision poly-HRP anti $\mathrm{Ms} / \mathrm{Rb} / \mathrm{Rt}$ for $30 \mathrm{~min}$, followed by staining with PowerDAB for $7 \mathrm{~min}$ and counterstaining with haematoxylin for $1 \mathrm{~min}$. All incubations were performed at room temperature.

Tissue blocks containing different tissue types were used as controls, with known staining patterns for both positive and negative stained tissues. 
Table 4.I Primary antibodies used for immunohistochemistry.

\begin{tabular}{|c|c|c|c|c|}
\hline Antibody & Clone & Manufacturer & Dilution & Antigen retrieval \\
\hline Calretinin & $5 \mathrm{~A} 5$ & Novocastra & $1: 25$ & EDTA pH 910 min at $96^{\circ} \mathrm{C}$ \\
\hline $\mathrm{HuC} / \mathrm{D}$ & $16 \mathrm{~A} 11$ & Molecular Probes & $1: 600$ & $\begin{array}{l}\text { Sodium citrate } 10 \mathrm{mM}(\mathrm{pH} 6.0) \\
30 \mathrm{~min} \text { at } 100^{\circ} \mathrm{C}\end{array}$ \\
\hline S100 & polyclonal & DAKO & $1: 10000$ & EDTA pH $910 \mathrm{~min}$ at $96^{\circ} \mathrm{C}$ \\
\hline CD117 & YR145 & Immunologic & $1: 200$ & None \\
\hline $\begin{array}{l}\alpha \text {-smooth muscle } \\
\text { actin ( } \alpha \text {-SMA) }\end{array}$ & $1 \mathrm{~A} 4$ & Sigma & $1: 7500$ & None \\
\hline Desmin & 33 & Biogenex & $1: 100$ & Citrate pH 6.730 min at $100^{\circ} \mathrm{C}$ \\
\hline
\end{tabular}

\section{Microscopic analysis}

Sections were evaluated blind to diagnosis and were then compared with those of the controls. The histology of the bowel wall was examined by H\&E. PAS was used to verify the presence or absence of polyglucosan inclusion bodies in the muscularis propria. The presence or absence of fibrosis was assessed in the submucosa, muscularis propria, and myenteric plexus on EVG-stained sections (Figure 4.3C).

Immunohistochemically stained sections were assessed by semiquantitative scoring using visual analysis to evaluate systematically the neuronal structures and smooth muscle layers.

The presence of neurons in ganglia was analysed on $\mathrm{HuC} / \mathrm{D}$ - and calretininstained sections. The number of neurons in relation to the present plexus was estimated in $\mathrm{HuC} / \mathrm{D}$ sections as follows: Firstly, the distribution of the neuronal network was evaluated on S100-stained sections. Subsequently, the number of neurons per ganglion was estimated in the $\mathrm{HuC} / \mathrm{D}$ staining in relation to this neuronal network and scored as 0 (no neurons), 1 (low neuronal density), and 2 (high neuronal density). The calretininstained sections were comparably rated: 0 (no neurons), 1 (on average less than one neuron per neuronal structure), and 2 (minimal one neuron per neuronal structure).

S100 was used to assess the distribution of nerve fibres (including nuclei of glial cells) in the submucosa, the myenteric plexus, and both muscle layers of the muscularis propria. The degree of distribution was scored as follows: 0 (no/low density) and 1 (high density of positive fibres).

The network of ICCs surrounding the myenteric plexus was estimated on CD117-stained sections as described earlier. ${ }^{[26]}$ The percentage of the circumference which is covered by CD117-positive cells was rated from 0 to $100 \%$ in $10 \%$ increments. Thus, a percentage of $0 \%$ represented no positive cells around the ganglia and in sections estimated as $100 \%$ the ganglia were completely surrounded by CD117-positive cells. Staining of mast cells was used as internal positive control.

$\alpha$-Smooth muscle actin ( $\alpha$-SMA) and desmin staining were used to assess the muscularis layers. Staining intensities of circular and longitudinal muscle layers were classified in two grades: 0 (no/weak) and 1 (strong staining intensity). Immunoreactivity 
within blood vessel walls and muscularis mucosae acted as internal reference for $\alpha$-SMA and desmin, respectively (grade 1).

Estimation of interobserver variation for the evaluation of staining resulted in interobserver agreement of $\alpha$-SMA 'almost perfect' ( $n=17), \mathrm{HuC} / \mathrm{D}$ submucosal plexus 'fair' (0.444), and myenteric plexus 'substantial' (0.609) $(\mathrm{n}=15)$. Of cases with multiple slides, the slide with the lowest overall scoring results was used for analysis.

\section{Statistical analysis}

Categorical variables were described by percentages. Differences between categorical variables were assessed by the chi-square test (likelihood ratio, exact p-values compared with the control group). Continuous variables were presented as means \pm standard deviation (SD). The Kruskal-Wallis test with post hoc pairwise comparisons using the Dunn-Bonferroni approach (SPSS procedure) was performed to compare the CD117 scores between groups, computing adjusted p-values corrected for multiple testing. A p-value of 0.05 was considered significant. Data were analysed by the IBM SPSS Statistics 20 Software (SPSS Inc., Chicago, IL, USA) and GraphPad Prism version 5.00 for Windows (GraphPad Software, San Diego, CA, USA).

\section{Results}

SB and SCI patients underwent surgery for several reasons, not limited to complaints that might be related to motility disorders. Therefore, the SB and SCI groups were each divided into two subgroups: first, a subgroup with known (severe) motility disorders which constituted the indication for bowel resection (from here referred to as 'symptomatic patients'; SB: $\mathrm{n}=7, \mathrm{SCI}: \mathrm{n}=21$ ) and, second, a subgroup in which motility disorders were not listed in the anonymised pathology reports, although possibly present in less severe form (from here referred to as 'asymptomatic patients'; SB: $n=6, S C I: n=13$ ). The latter subgroup included various indications for resection: carcinoma, diverticulitis, and inflammatory bowel disease (Crohn's disease, ulcerative colitis). Table 4.2 shows patient characteristics. An overview of the major results is given in Supplementary Table 4.1.

H\&E staining confirmed the normal histomorphology of control specimens. 
Table 4.2 Patient characteristics.

\begin{tabular}{|c|c|c|c|c|c|c|}
\hline & & \multirow{2}{*}{$\begin{array}{l}\text { Control } \\
(\mathrm{n}=16) \\
\end{array}$} & \multicolumn{2}{|l|}{ SB } & \multicolumn{2}{|l|}{ SCI } \\
\hline & & & $\begin{array}{l}\text { Asymptomatic } \\
(\mathrm{n}=6)\end{array}$ & $\begin{array}{l}\text { Symptomatic } \\
(\mathrm{n}=21)\end{array}$ & $\begin{array}{l}\text { Asymptomatic } \\
(\mathrm{n}=13)\end{array}$ & $\begin{array}{l}\text { Symptomatic } \\
(\mathrm{n}=7)\end{array}$ \\
\hline Gender, n (\%) & male & $6(37.5 \%)$ & $6(85.7 \%)$ & $0(0 \%)$ & $10(47.6 \%)$ & $7(35.8 \%)$ \\
\hline $\begin{array}{l}\text { Age, years } \\
(\text { mean } \pm \text { SD) }\end{array}$ & & $62.1 \pm 12.9$ & $15.1 \pm 14.1$ & $31.0 \pm 9.8$ & $55.4 \pm 13.6$ & $58.5 \pm 14.8$ \\
\hline \multirow{3}{*}{$\begin{array}{l}\text { Orientation } \\
\text { cross section, } \\
\mathrm{n}(\%)\end{array}$} & transversal & $16(100 \%)$ & $4(57.1 \%)$ & $5(83.3 \%)$ & $14(66.7 \%)$ & $7(53.8 \%)$ \\
\hline & longitudinal & $0(0 \%)$ & $1(14.3 \%)$ & $0(0 \%)$ & $4(19.0 \%)$ & $1(7.7 \%)$ \\
\hline & oblique & $0(0 \%)$ & $2(28.6 \%)$ & $1(16.7 \%)$ & $3(14.3 \%)$ & $5(38.5 \%)$ \\
\hline
\end{tabular}

$S B$, spina bifida, $S C I$, spinal cord injury, $S D$, standard deviation.

\section{Neuronal changes}

\section{Submucosal plexus}

The presence of neurons relative to nerve fibres in the submucosal plexus was evaluated by $\mathrm{HuC} / \mathrm{D}$ (all neurons) and calretinin staining (part of intrinsic primary afferent neurons, IPANs).

On $\mathrm{HuC} / \mathrm{D}$ staining (Figure $4.1 \mathrm{~A}-\mathrm{C}$ ), $31 \%$ of cases in the control group were scored as high neuronal density. The symptomatic and asymptomatic SB groups showed high neuronal density in $29 \%(\mathrm{p}=1.000)$ and $17 \%(\mathrm{p}=0.130)$ of patients, respectively. The symptomatic and asymptomatic SCI groups showed high neuronal density in, respectively, $29 \%(\mathrm{p}=1.000)$ and $9 \%(\mathrm{p}=0.350)$ of cases (Figure $4.1 \mathrm{~A})$.

In the control group, at least one calretinin-positive neuron per neuronal structure was present in $100 \%$ of cases. This was significantly lower in both the symptomatic and the asymptomatic SB group $(43 \%(\mathrm{p}=0.004)$ and $33 \%(\mathrm{p}=0.002)$, respectively). In SCI, only in the symptomatic subgroup was neuronal density decreased $(38 \%, \mathrm{p}<0.001)$, while in the asymptomatic group, $77 \%$ of cases showed at least one neuron per neuronal structure ( $\mathrm{p}=0.078$ ) (Figure 4.1D-F).

The density of nerve fibres was assessed by $\mathrm{S} 100$ staining (Figure 4.1G-I). Figure $4.1 \mathrm{G}$ demonstrates that in the control group, $94 \%$ of cases showed a high nerve fibre density. A high density was found in $86 \%$ of symptomatic SB cases ( $\mathrm{p}=1.000)$, in $50 \%$ of asymptomatic SB patients $(\mathrm{p}=0.046)$, in $62 \%$ of symptomatic SCI cases $(\mathrm{p}=0.050)$, and in $85 \%$ of asymptomatic SCI cases ( $\mathrm{p}=0.573)$. 

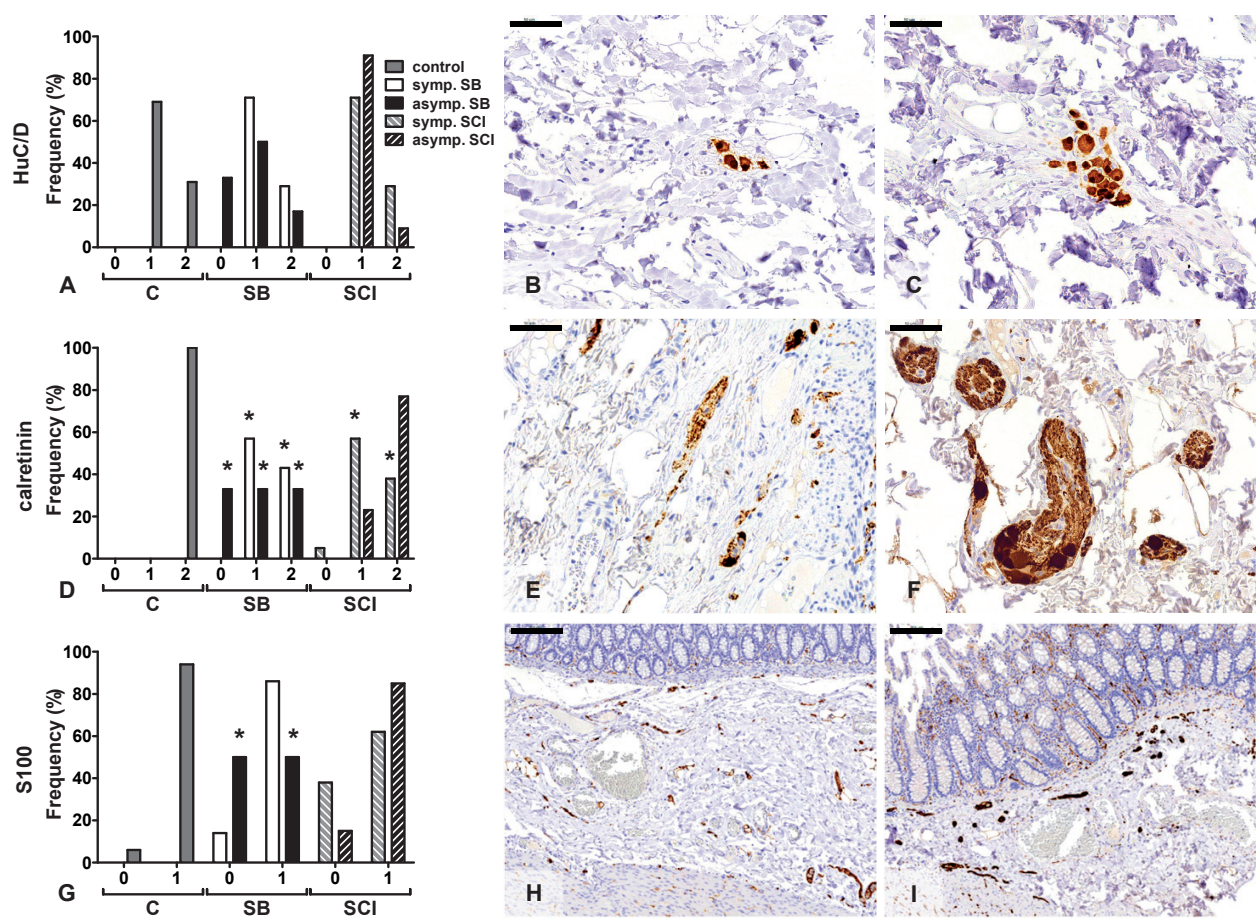

Figure 4.I Histological findings in the submucosal plexus of colon.

A) No significant differences were found between the HuC/D-stained sections. Most tissues showed low neuronal densities (grade 1) (B), while in some sections, high neuronal densities (grade 2) were found (C). D) Significantly less calretinin-positive neurons were found in both SB groups and the symptomatic SCI group compared with the control group. E) Section with neurons present, but less than one neuron per neuronal structure (grade 1). F) Minimal one neuron per neuronal structure is shown in this tissue (grade 2). G) Low nerve fibre densities (grade 0 ) were most often observed in the asymptomatic SB group. H, I) Illustration of low (grade 0) (H) and high (grade 1) (I) nerve fibre density. Numbers on the $x$-axis represent the semiquantitative scores. ${ }^{*} \mathrm{p}<0.05$ vs control. Scale bars $50 \mu \mathrm{m}$ (B, C, E, F) and $200 \mu \mathrm{m}(\mathrm{H}, \mathrm{I})$. C, control, $S B$, spina bifida, $S C I$, spinal cord injury.

\section{Myenteric plexus}

In the myenteric plexus, fewer neurons were observed in all SB and SCI groups (Figure 4.2A, D).

On the HuC/D-stained sections (all neurons) (Figure 4.2A-C), 88\% of cases in the control group were scored as high neuronal density. In the symptomatic and asymptomatic SB groups, 43\% ( $p=0.124)$ and 33\% ( $p=0.025)$ of cases, respectively, showed a high neuronal density. The symptomatic SCI group showed only in 33\% of cases a high density $(p=0.002)$, while $18 \%(p=0.001)$ of the asymptomatic SCI patients showed a high neuronal density (Figure 4.2A).

On calretinin staining (part of IPANs) (Figure 4.2D-F), neurons were present, but less than one neuron per neuronal structure in all control cases. The symptomatic 
SB group showed $86 \%(\mathrm{p}=0.304)$ and the asymptomatic SB group 67\% $(\mathrm{p}=0.065)$ of cases with on average less than one neuron per neuronal structure. In the symptomatic and asymptomatic SCI groups, 86\% ( $\mathrm{p}=0.495)$ and $85 \%(\mathrm{p}=0.192)$ of cases, respectively, showed presence of neurons, although less than one neuron per neuronal structure (Figure 4.2D).

The S100 stain showed a lower density of nerve fibres in SB and SCI compared with the control group (Figure 4.2G-I). All control cases showed a high nerve fibre density. High densities were significantly less found in the symptomatic SB group $(42.9 \%, \mathrm{p}=0.004)$, while $66.7 \%$ of asymptomatic SB cases showed high densities $(\mathrm{p}=0.065)$. In SCI, high density was significantly less often found in the symptomatic group $(67 \%, p=0.012)$, and $77 \%$ of asymptomatic patients showed a high density $(\mathrm{p}=0.078)$.

The presence of fibrosis in the myenteric plexus was assessed by EVG staining. As shown in Figure 4.3A, no significant differences between the control, SB, and SCI groups were found.
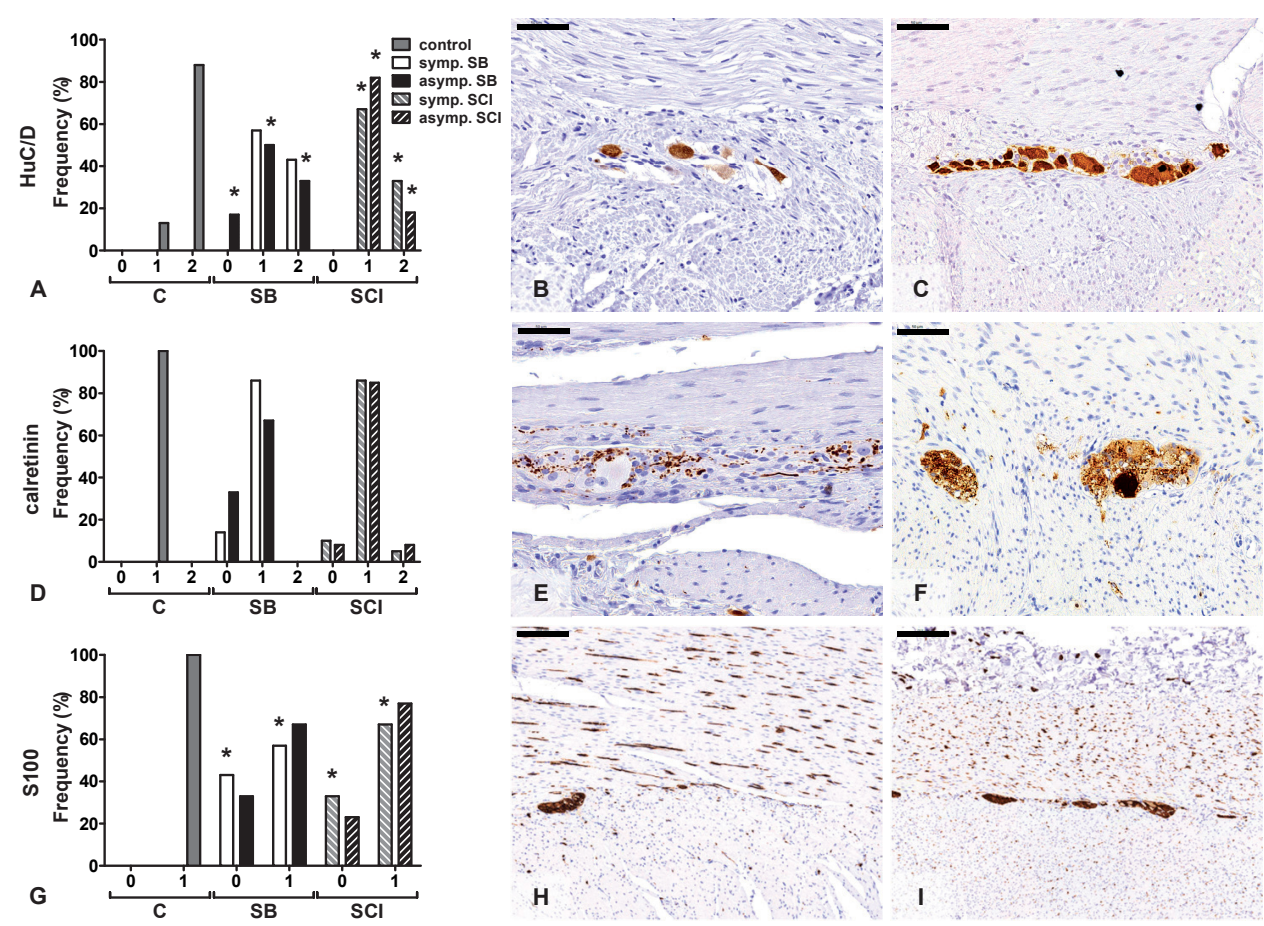

Figure 4.2 Histological findings in the myenteric plexus of colon.

A) $\mathrm{HuC} / \mathrm{D}$-positive neurons were significantly less often found in the asymptomatic SB group and both SCI groups compared with the control group. B, C) Examples of low (grade 1) (B) and high neuronal density (grade 2) (C). D) No differences in calretinin-positive neurons were found between groups. E) Tissue with no neurons per neuronal structure (grade 0). F) Example of tissue with neurons present, but less than one neuron per neuronal structure (grade 1). G) Lower nerve fibre densities were significantly more 
often found in the symptomatic SB and SCI groups compared with the control group. H, I) Representation of low (grade 0) (H) and high (grade 1) (I) nerve fibre densities. Numbers on the $x$-axis represent the semiquantitative scores. ${ }^{*} \mathrm{p}<0.05$ vs control. Scale bars $50 \mu \mathrm{m}$ (B, C, E, F) and $200 \mu \mathrm{m}(\mathrm{H}, \mathrm{I}) . C$, control, $S B$, spina bifida, $S C I$, spinal cord injury.

\section{Nerve fibres within muscularis propria}

In the circular muscle layer of the muscularis propria, a high nerve fibre density (S100 staining) was found in $100 \%$ of control cases. A significant decrease was seen in the symptomatic SB group (57\%, p $=0.020$ ), but not in the asymptomatic SB group nor in the SCI group. In the longitudinal muscle layer, no differences were observed (Supplementary Table 4.2).

\section{ICC changes}

Figure 4.4 shows the results of the estimation of the CD117-positive ICC network around the circumference of the myenteric plexus. Compared to the control group (mean $19.4 \pm 18.1 \%$ ), fewer CD117-positive cells were observed in the SB groups (symptomatic $5.7 \pm 5.3 \%, \mathrm{p}=0.510$ ( $\mathrm{p}=0.051$ without correction for multiple testing) and asymptomatic $5.0 \pm 5.5 \%, \mathrm{p}=0.374(\mathrm{p}=0.037$ without correction $)$ ) and $\mathrm{SCI}$ groups (symptomatic $5.2 \pm 6.0 \%, \mathrm{p}=0.029(\mathrm{p}=0.003$ without correction) and asymptomatic $6.9 \pm 7.5 \%, \mathrm{p}=0.343(\mathrm{p}=0.034$ without correction $))$.

\section{Smooth muscle changes}

The smooth muscle structure of the muscularis propria was assessed using $\alpha$-SMA and desmin staining. As shown in Supplementary Table 4.2, no significant differences were observed.

Figure 4.3A shows that fibrosis was not found in the circular muscle layer of the control group. Fibrosis was present in $29 \%(\mathrm{p}=0.083)$ of symptomatic and in $17 \%$ $(\mathrm{p}=0.273)$ of asymptomatic SB patients. The symptomatic and asymptomatic SCI groups showed fibrosis in $14 \%(\mathrm{p}=0.243)$ and $15 \%(\mathrm{p}=0.192)$ of cases, respectively. The longitudinal layer showed fibrosis in $13 \%$ of control cases. Fibrosis was significantly more often found in all SB groups (symptomatic 71\%, p $=0.011$, and asymptomatic $67 \%, \mathrm{p}=0.025$ ) and SCI groups (symptomatic 67\%, $\mathrm{p}=0.002$, and asymptomatic $85 \%, \mathrm{p}<0.001$ ) (Figure 4.3A-C).

The presence of polyglucosan inclusion bodies in the muscularis propria was assessed by PAS staining. ${ }^{[27,28]}$ No inclusion body myopathy was found in the smooth muscle of the control, SB, and SCI groups. 

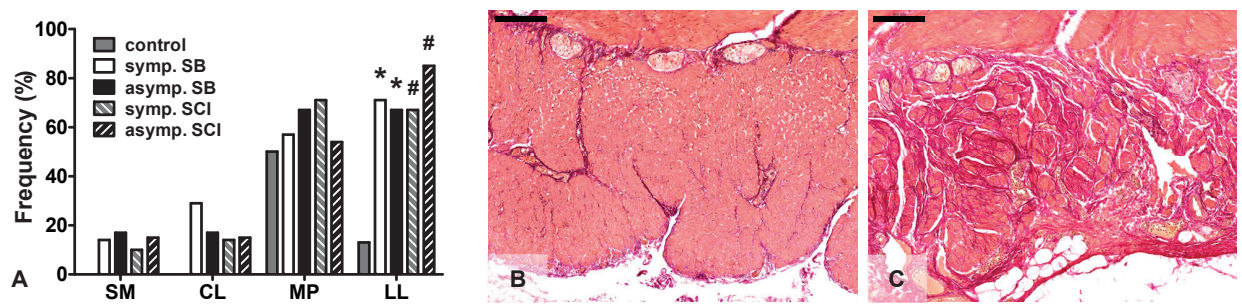

Figure 4.3 Presence of fibrosis in several layers of the bowel wall.

A) Fibrosis was significantly more often found in the longitudinal muscle layer of the SB and SCI groups.

B) Normal EVG staining pattern in the longitudinal layer of the muscularis propria (control). C) Fibrosis in the longitudinal muscle layer, showing excessive collagen accumulation. ${ }^{*} \mathrm{p}<0.05$ vs control, $\# \mathrm{p}<0.001$ vs control. Scale bars $200 \mu \mathrm{m}$. $S B$, spina bifida, $S C I$, spinal cord injury, $S M$, submucosa, $C L$, circular layer, $M P$, myenteric plexus, $L L$, longitudinal layer, $E V G$, elastic von Gieson.
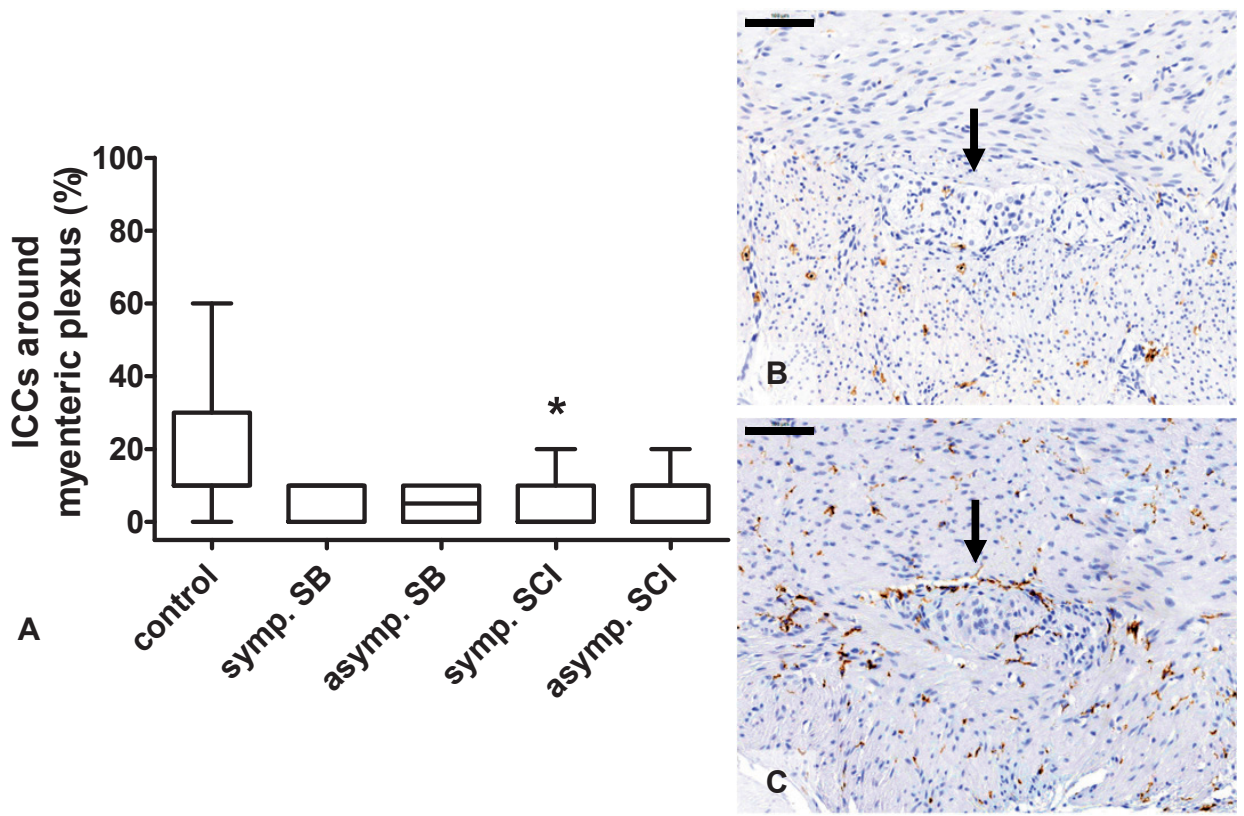

Figure 4.4 Percentage CDII7-positive cells (ICCs) around the circumference of the myenteric plexus (arrows).

A) Compared with the control group, less ICCs were found in the SB and SCI groups, significant in the symptomatic SCI group. B) Section without ICCs around the myenteric plexus. C) $60 \%$ of the ganglion is surrounded by ICCs. ${ }^{*} \mathrm{p}<0.05$ vs control. Scale bars $100 \mu \mathrm{m}$. ICC, interstitial cell of Cajal, SB, spina bifida, $S C I$, spinal cord injury. 


\section{Discussion}

The ENS of the colon plays an important role in the regulation of motility. Although the ENS can largely function independently, extrinsic input is needed to ensure proper bowel activity. ${ }^{[11,17]}$ The ENS is controlled by extrinsic innervation from the lower spinal cord, where parasympathetic fibres facilitate contraction of the colonic musculature and sympathetic fibres inhibit colon motility. ${ }^{[17,18]}$ Colon dysfunction following SCI can be distinguished into two main types, dependent on the level of the lesion: an upper motor neuron syndrome and a lower motor neuron syndrome. ${ }^{[4,29]}$ The upper motor neuron bowel is associated with spastic segmental contractions of the colon and decreased propulsive peristalsis, while the lower motor neuron bowel is associated with areflexia and reduced colon motility. Both syndromes result in constipation. ${ }^{[29]}$

Disruption of the extrinsic nerve fibres as in patients with SB and SCI results in changed ENS activity and subsequently in impaired motor function of the bowel. ${ }^{[2,}$ ${ }^{5,20,30]}$ We expected histological changes in the intrinsic neuromuscular apparatus of the colon, due to impaired extrinsic control. Main alterations were expected in the myenteric plexus, which controls motility. ${ }^{[10,11]}$ Histological evaluation of the neuromuscular structures (ENS, ICCs, smooth muscle) may provide insight in the pathophysiological mechanisms of neurogenic bowel in SB and SCI.

In the current study, we observed four changes in the neuromuscular apparatus. First, in analogy with two studies that investigated the effect of spinal cord and (parasympathetic) peripheral nerve lesions on the ENS, we did find loss of ganglion cells in the myenteric plexus of the colon. However, the submucosal plexus was considered to be normal ${ }^{[21,31]}$, which we confirmed in our study. Upon analysis of subsets of neurons, we observed loss of calretinin-positive neurons in the patient groups. Calretinin is a biomarker for a subpopulation of neurons including IPANs. ${ }^{[32]}$ IPANs are sensory neurons which play a role in the peristaltic reflex. ${ }^{[33]}$ In contrast, in the myenteric plexus, a reduction of the total neuron population was observed, both in the symptomatic and asymptomatic SB and SCI groups, which suggested that neuron loss could thus not solely be responsible for severe motility complaints.

Second, we found lower fibre density in the symptomatic but not in the asymptomatic groups. Since enteric glial cell nuclei are more prominent than nerve fibres in the healthy myenteric plexus, we believe that glial cells are lost in the symptomatic groups. Although the exact function of glial cells in intestinal motility is still unclear, they may have an important role in the physiological control of motility and in enteric neurotransmission. ${ }^{[13,34]}$ Loss of enteric glial cells might impair bowel motility, as has been shown both in humans ${ }^{[35]}$ and mice ${ }^{[36]}$. Our findings provide supportive evidence for a significant role of glial cells in the regulation of motility. Whether reduction of nerve fibres initiates neuronal loss or is a response to neuronal loss remains still unclear; further research on this mechanism is needed. 
Third, we found loss of the ICC network around the circumference of the myenteric plexus in almost all patients, although after performing a strict statistic test (Kruskal-Wallis) with correction for multiple testing, this was only significant in the symptomatic SCI group. The small subgroups resulted in low statistical power and lack of significance. Scoring of the density of the ICC network has shown good inter- and intraobserver agreement and reliability ${ }^{[26]}$, but the differences between our groups might be a result of rater variability. A previous study indicated that only tissues with completely absent ICCs may be diagnosed as abnormal in the colon. ${ }^{[2]}$ Several studies have shown that loss of ICCs in patients with motility problems is highly associated with changes of enteric nerves. ${ }^{[37]}$ Consequently, in most motility disorders, it is unclear whether ICC network disruption is primary or secondary to neuronal changes. ${ }^{[38]}$ We hypothesise that in our patients, ICC loss is indeed secondary to disruption of the extrinsic innervation in SB and SCI. Loss of the myenteric ICC network was shown in most patients, while approximately two thirds of this group revealed neuronal loss. Therefore, disruption of the ICC network may precede loss of enteric neurons.

Fourth, significantly more fibrosis was found in the longitudinal muscle layer in almost all patients. Although fibrosis in the longitudinal layer is frequently found in patients with visceral myopathy ${ }^{[23,39,40]}$, other muscle changes were not observed in our patients. Nevertheless, the presence of fibrosis might contribute to motility problems. Despite SB being a congenital and SCI an acquired disease, no clear differences between both conditions were found. All observed alterations in the neuromuscular apparatus may be explained as reactive to disrupted extrinsic innervation of the colon. This is supported by a study in rats with myelomeningocele, suggesting that the anorectal unit is normally developed. ${ }^{[41]}$ This has led to the hypothesis that loss of extrinsic innervation may result in trans-neuronal degeneration, although the exact mechanism in the bowel is currently unknown. ${ }^{[21,31]}$

Our study has some limitations. First, we aimed to study two large groups of patients to perform reliable comparisons which required collection of tissue blocks from SB and SCI patients on a national scale. We used proper cross sections of the entire colonic wall, from mucosa to serosa, and included cross sections in different directions to make the groups as large as possible. Nevertheless, the groups, notably the SB groups, were relatively small which resulted in low statistical power. Second, the anonymous character of the study resulted in lack of data on the severity of motility problems, the level of the lesion, the duration of the SCI, mobility of patients (bedridden, use of wheelchair), and medication. Patients in the symptomatic SB and SCI groups had severe motility problems, which was the indication for surgery. In contrast, of patients in the asymptomatic $S B$ and SCI groups, it remains unknown whether or not motility was normal. These patients might have suffered from motility problems in less severe form, which would be supported by the histological changes in these groups. Third, the mean age in the SB groups was much lower than that in the control group. Therefore, differences in enteric neuronal 
numbers between controls and SB patients might be underestimated as patients in the control group might have lost neurons due to ageing. ${ }^{[42]}$

In conclusion, we have shown that impaired extrinsic innervation affects neuromuscular structures which may contribute to decreased bowel motility. Major neuromuscular alterations in the colon of SB and SCI patients are loss of myenteric neurons and disruption of the ICC network around the myenteric plexus, accompanied by decreased nerve fibre density in the myenteric plexus. These changes might affect intestinal motility, already symptomatic in some patients and potentially symptomatic in others. In addition, nerve fibre density (glial cells) in the myenteric plexus was significantly decreased in the symptomatic SB and SCI groups, which may be the reason for the major motility problems found in these patients. 


\section{References}

1. Verhoef M, Barf HA, Post MWM, et al. Secondary impairments in young adults with spina bifida. Dev Med Child Neurol, 2004. 46(6): p. 420-427.

2. Ebert E. Gastrointestinal involvement in spinal cord injury: a clinical perspective. Journal of gastrointestinal and liver diseases, 2012. 21(1): p. 75-82.

3. Krogh K, Nielsen J, Djurhuus JC, et al. Colorectal function in patients with spinal cord lesions. Diseases of the Colon \& Rectum, 1997. 40(10): p. 1233-1239.

4. Stiens SA, Bergman SB, Goetz LL. Neurogenic bowel dysfunction after spinal cord injury: clinical evaluation and rehabilitative management. Arch Phys Med Rehabil, 1997. 78(3 Suppl): p. S86102.

5. Webb TS. Optimizing health care for adults with spina bifida. Dev Disabil Res Rev, 2010. 16(1): p. 76-81.

6. Ravelli AM, Milla PJ. Vomiting and gastroesophageal motor activity in children with disorders of the central nervous system. J Pediatr Gastroenterol Nutr, 1998. 26(1): p. 56-63.

7. Liptak GS, El Samra A. Optimizing health care for children with spina bifida. Dev Disabil Res Rev, 2010. 16(1): p. 66-75.

8. Pigeon N, Leroi AM, Devroede G, et al. Colonic transit time in patients with myelomeningocele. Neurogastroenterol Motil, 1997. 9(2): p. 63-70.

9. Faaborg PM, Christensen P, Finnerup N, et al. The pattern of colorectal dysfunction changes with time since spinal cord injury. Spinal Cord, 2007. 46(3): p. 234-238.

10. Furness JB. The Enteric Nervous System. Second ed. 2008, Oxford: Wiley-Blackwell.

11. Wood JD. Enteric Nervous System: Physiology, in Encyclopedia of Neuroscience, Squire LR, Editor. 2009, Academic Press: Oxford. p. 1103-1113.

12. Benarroch EE. Enteric nervous system: functional organization and neurologic implications. Neurology, 2007. 69(20): p. 1953-1957.

13. Sharkey KA. Emerging roles for enteric glia in gastrointestinal disorders. J Clin Invest, 2015. 125(3): p. 918-925.

14. Huizinga JD, Martz S, Gil V, et al. Two independent networks of interstitial cells of cajal work cooperatively with the enteric nervous system to create colonic motor patterns. Front Neurosci, 2011. 5: p. 93.

15. Blair PJ, Rhee PL, Sanders KM, et al. The significance of interstitial cells in neurogastroenterology. J Neurogastroenterol Motil, 2014. 20(3): p. 294-317.

16. Komuro T. Atlas of Interstitial Cells of Cajal in the Gastrointestinal Tract. 2012, Dordrecht: Springer Science \& Business Media.

17. Boron WF, Boulpaep EL. Medical physiology: a cellular and molecular approach. Second ed. 2012, Philadelphia: Elsevier Health Sciences.

18. Camilleri M, Ford MJ. Review article: colonic sensorimotor physiology in health, and its alteration in constipation and diarrhoeal disorders. Aliment Pharmacol Ther, 1998. 12(4): p. 287-302.

19. Inskip JA, Ramer LM, Ramer MS, et al. Autonomic assessment of animals with spinal cord injury: 
tools, techniques and translation. Spinal Cord, 2009. 47(1): p. 2-35.

20. Camilleri M, Bharucha AE. Gastrointestinal dysfunction in neurologic disease. Semin Neurol, 1996. 16(3): p. 203-216.

21. Devroede G, Lamarche J. Functional importance of extrinsic parasympathetic innervation to the distal colon and rectum in man. Gastroenterology, 1974. 66(2): p. 273-280.

22. Bernardini N, Ippolito C, Segnani C, et al. Histopathology in gastrointestinal neuromuscular diseases: methodological and ontological issues. Adv Anat Pathol, 2013. 20(1): p. 17-31.

23. Knowles CH, De Giorgio R, Kapur RP, et al. Gastrointestinal neuromuscular pathology: guidelines for histological techniques and reporting on behalf of the Gastro 2009 International Working Group. Acta Neuropathol, 2009. 118(2): p. 271-301.

24. Casparie M, Tiebosch AT, Burger G, et al. Pathology databanking and biobanking in The Netherlands, a central role for PALGA, the nationwide histopathology and cytopathology data network and archive. Cell Oncol, 2007. 29(1): p. 19-24.

25. Code of Conduct of the Dutch federation of Medical Scientific Societies. [Internet]. Available from: http://www.federa.org. Accessed 8 Dec 2014.

26. den Braber-Ymker M, Heijker S, Lammens M, et al. Practical and reproducible estimation of myenteric interstitial cells of Cajal in the bowel for diagnostic purposes. Neurogastroenterol Motil, 2016. 28(8): p. 1261-1267.

27. Knowles CH, De Giorgio R, Kapur RP, et al. The London Classification of gastrointestinal neuromuscular pathology: report on behalf of the Gastro 2009 International Working Group. Gut, 2010. 59(7): p. 882-887.

28. Knowles CH, Nickols CD, Feakins R, et al. A systematic analysis of polyglucosan bodies in the human gastrointestinal tract in health and disease. Acta Neuropathol, 2003. 105(4): p. 410-413.

29. Hermann J. Pathophysiology Prevention and Management of Chronic Neurogenic Constipation in Adults. Science Journal of Clinical Medicine, 2013. 2(4): p. 122-128.

30. Chung EAL, Emmanuel AV. Gastrointestinal symptoms related to autonomic dysfunction following spinal cord injury, in Progress in Brain Research, Weaver LC and Polosa C, Editors. 2005, Elsevier. p. 317-333.

31. Devroede G, Arhan P, Duguay C, et al. Traumatic constipation. Gastroenterology, 1979. 77(6): p. $1258-1267$.

32. Furness JB. Enteric Nervous System: Neural Circuits and Chemical Coding, in Encyclopedia of Neuroscience, Squire LR, Editor. 2009, Academic Press: Oxford. p. 1089-1095.

33. Clerc N, Furness JB. Enteric Nervous System: Sensory Pathways, in Encyclopedia of Neuroscience, Squire LR, Editor. 2009, Academic Press: Oxford. p. 1115-1120.

34. Neunlist M, Rolli-Derkinderen M, Latorre R, et al. Enteric glial cells: recent developments and future directions. Gastroenterology, 2014. 147(6): p. 1230-1237.

35. Bassotti G, Villanacci V, Cathomas G, et al. Enteric neuropathology of the terminal ileum in patients with intractable slow-transit constipation. Human Pathology, 2006. 37(10): p. 1252-1258.

36. Aubé AC, Cabarrocas J, Bauer J, et al. Changes in enteric neurone phenotype and intestinal functions in a transgenic mouse model of enteric glia disruption. Gut, 2006. 55(5): p. 630-637. 


\section{Chapter 4}

37. Streutker CJ, Huizinga JD, Driman DK, et al. Interstitial cells of Cajal in health and disease. Part I: normal ICC structure and function with associated motility disorders. Histopathology, 2007. 50(2): p. 176-189.

38. Huizinga JD, Lammers WJEP. Gut peristalsis is governed by a multitude of cooperating mechanisms. Am J Physiol Gastrointest Liver Physiol, 2009. 296(1): p. G1-8.

39. Odze RD, Goldblum JR. Surgical pathology of the GI tract, liver, billiary tract, and pancreas. 2nd ed, ed. Odze RD. 2009, Philadelphia: Elsevier Health Sciences. 1368.

40. Mann SD, Debinski HS, Kamm MA. Clinical characteristics of chronic idiopathic intestinal pseudo-obstruction in adults. Gut, 1997. 41(5): p. 675-681.

41. Danzer E, Radu A, Robinson LE, et al. Morphologic analysis of the neuromuscular development of the anorectal unit in fetal rats with retinoic acid induced myelomeningocele. Neurosci Lett, 2008. 430(2): p. 157-162.

42. Saffrey MJ. Cellular changes in the enteric nervous system during ageing. Developmental Biology, 2013. 382(1): p. 344-355. 


\section{Supplementary material}

Supplementary Table 4.I Overview of the main conclusions of chapter 4.

Differences compared with the control group are represented as no difference $(=)$, decrease/increase $(\downarrow / \uparrow)$, or significant decrease/increase $(\downarrow \downarrow / \uparrow \uparrow)$.

\begin{tabular}{|c|c|c|c|c|}
\hline & \multicolumn{2}{|l|}{ SB } & \multicolumn{2}{|l|}{ SCI } \\
\hline & symptomatic & asymptomatic & symptomatic & asymptomatic \\
\hline \multicolumn{5}{|l|}{ Submucosal plexus } \\
\hline Total neurons (HuC/D) & $=$ & $=$ & $=$ & $\downarrow$ \\
\hline Calretinin positive neurons & $\downarrow \downarrow$ & $\downarrow \downarrow$ & $\downarrow \downarrow$ & $\downarrow$ \\
\hline Nerve fibres & $=$ & $\downarrow$ & $\downarrow$ & $=$ \\
\hline \multicolumn{5}{|l|}{ Myenteric plexus } \\
\hline Total neurons $(\mathrm{HuC} / \mathrm{D})$ & $\downarrow$ & $\downarrow$ & $\downarrow \downarrow$ & $\downarrow \downarrow$ \\
\hline Calretinin positive neurons & $=$ & $\downarrow$ & $=$ & $=$ \\
\hline Nerve fibres & $\downarrow \downarrow$ & $\downarrow$ & $\downarrow \downarrow$ & $\downarrow$ \\
\hline ICC network & $\downarrow$ & $\downarrow$ & $\downarrow \downarrow$ & $\downarrow$ \\
\hline \multicolumn{5}{|l|}{ Circular muscle } \\
\hline$\alpha$-SMA & $\downarrow$ & $=$ & $=$ & $=$ \\
\hline Desmin & $=$ & $=$ & $=$ & $=$ \\
\hline Fibrosis & $=$ & $=$ & $=$ & $=$ \\
\hline Nerve fibres & $\downarrow \downarrow$ & $=$ & $=$ & $=$ \\
\hline \multicolumn{5}{|l|}{ Longitudinal muscle } \\
\hline$\alpha$-SMA & $\downarrow$ & $=$ & $=$ & $=$ \\
\hline Desmin & $=$ & $=$ & $=$ & $=$ \\
\hline Fibrosis & $\uparrow \uparrow$ & $\uparrow \uparrow$ & 个个 & $\uparrow \uparrow$ \\
\hline Nerve fibres & $=$ & $=$ & $=$ & $=$ \\
\hline
\end{tabular}




\section{Chapter 4}

Supplementary Table 4.2 Semiquantitative evaluation of immunohistochemical markers in the different layers of colonic muscularis propria in controls and in patients with spina bifida (SB) or spinal cord injury (SCI).

Expression of $\alpha$-SMA is more often lower in the circular and longitudinal muscle layer of the symptomatic SB group (not significant). In the circular layer, lower nerve fibre densities (S100) are most frequently found in the symptomatic SB group.

\begin{tabular}{|c|c|c|c|c|c|c|c|c|c|c|}
\hline \multirow[b]{3}{*}{ Antibody } & \multirow[b]{3}{*}{ Score } & \multirow{3}{*}{$\begin{array}{l}\text { Control } \\
\mathrm{n}(\%)\end{array}$} & \multicolumn{4}{|l|}{ SB } & \multicolumn{4}{|l|}{ SCI } \\
\hline & & & \multicolumn{2}{|c|}{ symptomatic } & \multicolumn{2}{|c|}{ asymptomatic } & \multicolumn{2}{|c|}{ symptomatic } & \multicolumn{2}{|c|}{ asymptomatic } \\
\hline & & & n (\%) & $\mathrm{p}$-value & n (\%) & $\mathrm{p}$-value & n (\%) & $\begin{array}{l}\text { p-val- } \\
\text { ue }\end{array}$ & n (\%) & p-value \\
\hline \multirow{2}{*}{$\alpha$-SMA CL } & 0 & $\begin{array}{l}1 \\
(6.2 \%)\end{array}$ & $\begin{array}{l}3 \\
(42.9 \%)\end{array}$ & \multirow{2}{*}{.067} & & \multirow{2}{*}{1.000} & & \multirow{2}{*}{.432} & & \multirow{2}{*}{1.000} \\
\hline & 1 & $\begin{array}{l}15 \\
(93.8 \%)\end{array}$ & $\begin{array}{l}4 \\
(57.1 \%)\end{array}$ & & $\begin{array}{l}7 \\
(100 \%)\end{array}$ & & $\begin{array}{l}21 \\
(100 \%)\end{array}$ & & $\begin{array}{l}13 \\
(100 \%)\end{array}$ & \\
\hline \multirow{2}{*}{$\alpha$-SMA LL } & 0 & & $\begin{array}{l}2 \\
(28.6 \%)\end{array}$ & \multirow{2}{*}{.083} & & & & & & \\
\hline & 1 & $\begin{array}{l}16 \\
(100 \%)\end{array}$ & $\begin{array}{l}5 \\
(71.4 \%)\end{array}$ & & $\begin{array}{l}7 \\
(100 \%)\end{array}$ & & $\begin{array}{l}21 \\
(100 \%)\end{array}$ & & $\begin{array}{l}13 \\
(100 \%)\end{array}$ & \\
\hline \multirow[b]{2}{*}{ desmin CL } & 0 & & & & & & & & & \\
\hline & 1 & $\begin{array}{l}16 \\
(100 \%)\end{array}$ & $\begin{array}{l}7 \\
(100 \%)\end{array}$ & & $\begin{array}{l}6 \\
(100 \%)\end{array}$ & & $\begin{array}{l}17 \\
(100 \%)\end{array}$ & & $\begin{array}{l}14 \\
(100 \%)\end{array}$ & \\
\hline \multirow{2}{*}{ desmin LL } & 0 & & & & $\begin{array}{l}1 \\
(16.7 \%)\end{array}$ & \multirow[b]{2}{*}{-.273} & & & $\begin{array}{l}1 \\
(7.1 \%)\end{array}$ & \multirow[b]{2}{*}{-.467} \\
\hline & 1 & $\begin{array}{l}16 \\
(100 \%)\end{array}$ & $\begin{array}{l}7 \\
(100 \%)\end{array}$ & & $\begin{array}{l}5 \\
(83.3 \%)\end{array}$ & & $\begin{array}{l}17 \\
(100 \%)\end{array}$ & & $\begin{array}{l}13 \\
(92.0 \%)\end{array}$ & \\
\hline \multirow{2}{*}{ S100 CL } & 0 & & $\begin{array}{l}3 \\
(42.9 \%)\end{array}$ & \multirow{2}{*}{$.020^{*}$} & & & $\begin{array}{l}2 \\
(9.5 \%)\end{array}$ & \multirow{2}{*}{-.495} & $\begin{array}{l}1 \\
(7.1 \%)\end{array}$ & \multirow{2}{*}{-.467} \\
\hline & 1 & $\begin{array}{l}16 \\
(100 \%)\end{array}$ & $\begin{array}{l}4 \\
(57.1 \%)\end{array}$ & & $\begin{array}{l}7 \\
(100 \%)\end{array}$ & & $\begin{array}{l}19 \\
(90.5 \%)\end{array}$ & & $\begin{array}{l}13 \\
(92.9 \%)\end{array}$ & \\
\hline \multirow{2}{*}{ S100 LL } & 0 & $\begin{array}{l}15 \\
(93.8 \%)\end{array}$ & $\begin{array}{l}5 \\
(71.4 \%)\end{array}$ & \multirow{2}{*}{.526} & $\begin{array}{l}7 \\
(100 \%)\end{array}$ & \multirow{2}{*}{1.000} & $\begin{array}{l}17 \\
(81.0 \%)\end{array}$ & \multirow{2}{*}{.364} & $\begin{array}{l}11 \\
(78.6 \%)\end{array}$ & \multirow{2}{*}{-.315} \\
\hline & 1 & $\begin{array}{l}1 \\
(6.2 \%)\end{array}$ & $\begin{array}{l}2 \\
(28.6 \%)\end{array}$ & & & & $\begin{array}{l}4 \\
(19.0 \%)\end{array}$ & & $\begin{array}{l}3 \\
(21.4 \%)\end{array}$ & \\
\hline
\end{tabular}

${ }^{*} \mathrm{p}<0.05$ vs control. $\alpha$-SMA, $\alpha$-smooth muscle actin, $C L$, circular layer, $L L$, longitudinal layer, $S B$, spina bifida, $S C I$, spinal cord injury. 
Neuromuscular changes in colon of spina bifida and spinal cord injury 


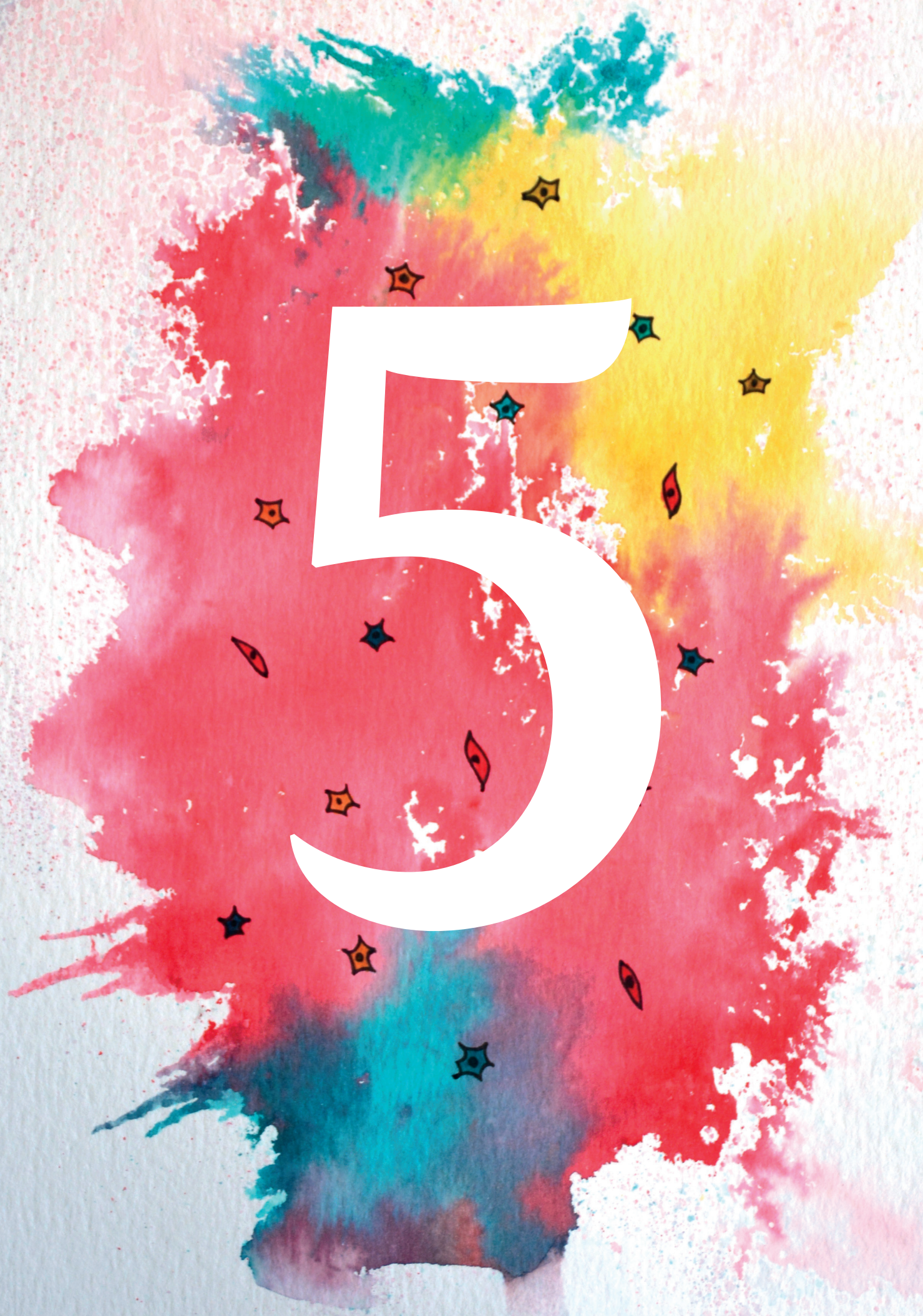




\section{Chapter 5}

\section{Intestinal involvement in amyloidosis is a sequential process}

M. den Braber-Ymker ${ }^{1}$, S. Heijker ${ }^{2}$, M. Lammens ${ }^{1,3,4}$, S. Croockewit ${ }^{5}$, I.D. Nagtegaal ${ }^{1}$

${ }^{1}$ Department of Pathology, Radboud University Medical Center, Nijmegen, The Netherlands ${ }^{2}$ Department of Pathology, Netherlands Cancer Institute-Antoni van Leeuwenhoek, Amsterdam, The Netherlands ${ }^{3}$ Department of Pathology, Antwerp University Hospital, University of Antwerp, Edegem, Belgium ${ }^{4}$ MIPRO, University of Antwerp, Antwerp, Belgium

${ }^{5}$ Department of Hematology, Radboud University Medical Center, Nijmegen, The Netherlands

Neurogastroenterol Motil, 2018. 30(12):e13469 https://doi.org/10.1111/nmo.13469 


\section{Abstract}

Gastrointestinal amyloidosis causes dysmotility. A comprehensive histological analysis to explain these symptoms is lacking. Therefore, we systematically examined histological features of intestinal dysmotility in patients with AL and AA amyloidosis, compared to controls.

Autopsy tissue material from small bowel and colon was used for histological (semiquantitative) evaluation of the mucosa, blood vessels, muscular layers, enteric nervous system (ENS) and the interstitial cells of Cajal (ICC), using haematoxylin and eosin, periodic acid Schiff, elastic von Gieson and Congo Red staining, and immunohistochemistry with $\alpha$-smooth muscle actin, HuC/D, S100 and CD117 antibodies, according to guidelines of the Gastro 2009 International Working Group.

Amyloid deposits were present in the vascular walls of all amyloidosis patients. In the mucosa, amyloid was found in $67 \%$ of AA patients. The muscular layers were involved in $64 \%$ of amyloidosis patients, most prominent in AA patients, associated with the presence of polyglucosan inclusion bodies, but not with either abnormal $\alpha$-actin patterns or fibrosis. Amyloid in the muscularis propria surrounding the myenteric plexus was found, but not inside the myenteric plexus. These deposits might be related to loss of the ICC network, but there was no association with decreased neuronal or nerve fibre density.

We hypothesise that intestinal dysmotility in amyloidosis patients is a sequential process: amyloid deposition starts in the vasculature, followed by involvement of the muscular layers, ICC loss and potentially affect the myenteric plexus. This final stage may be accompanied by clinical symptoms of severe intestinal dysmotility. 


\section{Introduction}

Amyloidosis is a diverse group of disorders characterised by extracellular abnormal fibrillar protein deposits in various tissues, including the gastrointestinal (GI) tract, resulting in tissue damage caused by several mechanisms. ${ }^{[1,2]}$

Amyloid deposits can be found in all layers and structures of the bowel wall, including musculature, vasculature and neural structures. In the muscles this leads to pressure atrophy, while in vessel walls amyloid deposits result in ischaemia and infarction. ${ }^{[3]}$ Different types of amyloidosis show different damage patterns. ${ }^{[4]} \mathrm{AL}$ amyloidosis, i.e. light chain amyloid associated with B cell dyscrasias, supposedly shows a preference for the muscularis mucosae and muscularis propria, resulting in myopathy ${ }^{[5-7]}$, which causes constipation, mechanical obstruction or chronic intestinal pseudo-obstruction. ${ }^{[-8]}$ In AA amyloidosis, associated with inflammatory disorders, fine granular amyloid deposits are mainly found in the lamina propria (mucosa), resulting into diarrhoea and malabsorption, and in the myenteric plexus, leading to neuropathy ${ }^{[5,6,8]}$ Hence, both myopathy and neuropathy may be the underlying cause of intestinal dysmotility. Amyloidosis of the GI tract can therefore be classified as a secondary intestinal motility disorder. ${ }^{[9]}$ Intestinal dysmotility is present in $10-70 \%$ of patients with AL and AA amyloidosis ${ }^{[10]}$, however, the literature is mainly restricted to case reports, rarely larger patient series are described.

Guidelines for studying the intestinal neuromusculature have been presented by the Gastro 2009 International Working Group. ${ }^{[9]}$ The present study is the first study using these guidelines for histological evaluation of tissues from amyloidosis patients. The aim was to perform a systematic explorative study of the histological characteristics of intestinal dysmotility in amyloidosis patients, in order to develop insights in the mechanisms.

\section{Methods}

\section{Subjects}

Archived autopsy formalin-fixed paraffin-embedded tissue samples of segments of small bowel $(n=10)$ and colon $(n=12)$ were obtained from amyloidosis patients selected for presence of amyloid deposits in the intestinal wall. Complete autopsy records were available in all patients, patient dossiers were available in 11 of 14 patients. Amyloidosis patients were included in the AL or AA group, based on the diagnosis described in the autopsy reports and patients dossiers. Amyloid A antibody (Table 5.1) was used to confirm the diagnosis AA or non-AA amyloidosis. Control autopsy material of ileum $(\mathrm{n}=22)$ and colon $(\mathrm{n}=23)$ was obtained from patients without intestinal motility problems (according to the pathology reports) and considered as normal. 
Chapter 5

Table 5.I Primary antibodies used for immunohistochemistry.

\begin{tabular}{lllll}
\hline Antibody & Clone & Manufacturer & Dilution & Antigen retrieval \\
\hline Amyloid A & Reu-86.2 & Monosan, Sanbio & $1: 80$ & None \\
HuC/D & 16A11 & Molecular Probes & $1: 600$ & $\begin{array}{l}\text { Sodium citrate } 10 \mathrm{mM}(\mathrm{pH} 6.0) 30 \\
\text { min at } 100^{\circ} \mathrm{C}\end{array}$ \\
S100 & polyclonal & DAKO & $1: 10000$ & EDTA pH 910 min at $96^{\circ} \mathrm{C}$ \\
CD117 & YR145 & Immunologic & $1: 200$ & None \\
$\begin{array}{l}\alpha-\text {-smooth muscle } \\
\text { actin }(\alpha-S M A)\end{array}$ & 1A4 & Sigma & $1: 7500$ & None \\
\hline
\end{tabular}

The study was approved by the local ethics committee (reference number 2014-1256). Samples were obtained in accordance with the Code of Conduct of the Federation of Medical Scientific Societies in the Netherlands. ${ }^{[1]}$

\section{Tissue preparation}

Sections were cut from formalin-fixed paraffin-embedded full-thickness tissue blocks for conventional histology or immunohistochemistry. Sections were deparaffinised, rehydrated in xylene and ethanol series, and rinsed in tap water by standard protocol.

\section{Histological staining}

Sections of $4 \mu \mathrm{m}$ were used for haematoxylin and eosin (H\&E) and periodic acid Schiff (PAS) staining. Elastic von Gieson (EVG) and Congo Red staining were performed on $6-\mu \mathrm{m}$ sections. Tissues were stained by standard protocols in a Medite TST 30 stainer (Klinipath, Duiven, The Netherlands).

\section{Immunohistochemistry}

Immunohistochemical staining was performed on 4- $\mu \mathrm{m}$ sections. Antibodies, suppliers, and dilutions are listed in Table 5.1.

For $\mathrm{HuC} / \mathrm{D}$ staining, antigen retrieval was performed in sodium citrate $(\mathrm{pH}$ 6) at $100{ }^{\circ} \mathrm{C}$ for $30 \mathrm{~min}$. Subsequently, endogenous peroxidase was blocked with $3 \%$ hydrogen peroxide in PBS for $20 \mathrm{~min}$. Sections were then rinsed in PBS and incubated with primary antibody anti-HuC/D at $4{ }^{\circ} \mathrm{C}$ overnight. After washing in PBS, sections were incubated for 30 min with a secondary antibody (Powervision poly-HRP anti $\mathrm{Ms} / \mathrm{Rb} / \mathrm{Rt} \mathrm{IgG}$, Immunologic, Duiven, The Netherlands) at room temperature. Subsequently, sections were rinsed in PBS and immunoreactivity was developed with PowerDAB (Immunologic) for $7 \mathrm{~min}$ at room temperature. Finally, sections were rinsed in tap water, counterstained with haematoxylin, rinsed in tap water, dehydrated in $100 \%$ ethanol and xylene, and mounted with Permount. 
The other immunohistochemical staining reactions were performed in an automated LabVision Autostainer 480 (Klinipath, Duiven, The Netherlands). The method used for antigen retrieval depended on the antibody (Table 5.1). Endogenous peroxidase was blocked with 3\% hydrogen peroxide in methanol for 10 min. Sections were incubated with primary antibody for $60 \mathrm{~min}$. Subsequently, the sections were incubated with Powervision poly-HRP anti $\mathrm{Ms} / \mathrm{Rb} / \mathrm{Rt}$ for $30 \mathrm{~min}$, followed by staining with PowerDAB for $7 \mathrm{~min}$ and counterstaining with haematoxylin for $1 \mathrm{~min}$. All incubations were performed at room temperature.

Tissue blocks containing different tissue types were used as controls for the immunohistochemical staining reactions, with known staining patterns for both positive and negative stained tissues.

\section{Microscopic analysis}

Sections were evaluated blind to diagnosis by two observers. The histology of the bowel wall was examined by H\&E. PAS was used to verify the presence or absence of polyglucosan inclusion bodies in the muscularis propria. The presence or absence of fibrosis was assessed in the submucosa, muscularis propria, and myenteric plexus on EVG-stained sections. Congo Red stained sections were used to evaluate the presence of amyloid deposits in the mucosa, submucosa, muscularis propria, and vascular walls.

Immunohistochemically stained sections were assessed by semiquantitative scoring using visual analysis to evaluate systematically the smooth muscle layers and neuronal structures, as previously described ${ }^{[12]} \alpha$-Smooth muscle actin ( $\alpha$-SMA) staining was used to assess the muscular layers. Staining intensities of circular and longitudinal muscle layers were classified in two grades: 0 (no/weak) and 1 (strong staining intensity). ${ }^{[12]}$ The presence of neurons in ganglia was analysed on $\mathrm{HuC} / \mathrm{D}$-stained sections. The number of neurons in relation to the present plexus was estimated in $\mathrm{HuC} / \mathrm{D}$ sections as follows: 0 (no neurons), 1 (low neuronal density), and 2 (high neuronal density). S100 was used to assess the distribution of nerve fibres (including nuclei of glial cells) in the submucosa, the myenteric plexus, and both muscle layers of the muscularis propria. The degree of distribution was scored as follows: 0 (no/low density) and 1 (high density of positive fibres). ${ }^{[12]}$ The network of interstitial cells of Cajal (ICCs) surrounding the myenteric plexus was estimated on CD117-stained sections as described earlier. ${ }^{[13]}$ The percentage of the circumference which is covered by CD117-positive cells was rated from 0 to $100 \%$ in $10 \%$ increments. Thus, a percentage of $0 \%$ represented no positive cells around the ganglia and in sections estimated as $100 \%$ the ganglia were completely surrounded by CD117-positive cells. 


\section{Statistical analysis}

Categorical variables were described by percentages. Differences between categorical variables were assessed by the chi-square test (likelihood ratio, exact p-values compared with the control group). Continuous variables were presented as means \pm standard deviation (SD). The Mann-Whitney test was performed to compare the CD117 scores between groups. A p-value of 0.05 was considered significant. Data were analysed by the IBM SPSS Statistics 22 Software (SPSS Inc., Chicago, IL, USA), GraphPad Prism version 5.00 for Windows (GraphPad Software, San Diego, CA, USA), and RStudio version 1.1.419 (RStudio, Inc., Boston, MA).

\section{Results}

\section{Patients}

Tissue material of $8 \mathrm{AL}$ amyloidosis patients (mean age $66 \pm 13$ years, $88 \%$ male), 6 AA amyloidosis patients (mean age $57 \pm 20$ years, $100 \%$ male) and 26 control patients (mean age $65 \pm 14$ years, 58\% male) was evaluated in this study. The AL amyloidosis group $(\mathrm{n}=8)$ consisted of 5 small bowel and 7 colon cases, from 4 patients tissues of both locations were analysed. The AA amyloidosis group $(n=6)$ included 5 small bowel and 5 colon tissues, from 4 patients tissues of both locations were assessed. In the control group $(n=26), 22$ small bowel and 23 colon cases were included.

Among the patients with amyloidosis, gastrointestinal bleeding was reported in two AL patients and in two AA patients. Ileus was reported in one AL and three AA cases, of which one patient with severe dysmotility of the oesophagus. Clinical symptoms of gastrointestinal involvement of the other patients were absent or unknown (Table 5.2).

\section{Amyloid deposition}

The presence of amyloid deposits in the mucosa, muscularis mucosae, muscularis propria, myenteric plexus and vascular walls was assessed by Congo Red staining (Figure 5.1). Overall, amyloid deposits were more frequently found in both the mucosa and muscularis mucosae of AA amyloidosis compared to AL amyloidosis, no amyloid was present in the control group. There was no difference between small bowel and colon tissues of the same patients. Amyloid deposits were present in all layers of the intestinal wall, except inside the myenteric plexus. All cases with amyloidosis showed amyloid deposits in the vascular walls, most prominent in the submucosa. In three patients extravasation of erythrocytes was present in the submucosa (one colon AL, two small bowel AA), but there were no signs of chronic blood loss (hemosiderin deposits). Mucosal presence of amyloid was most pronounced in the colon of AA patients $(80 \%$ versus $0 \%$ in AL patients, $\mathrm{p}=0.015)$. A comparable trend was found in the small bowel $(60 \%$ versus $0 \%, \mathrm{p}=0.167)$. No other differences were shown between the AL and AA groups (Figure 5.1). 
Intestinal involvement in amyloidosis

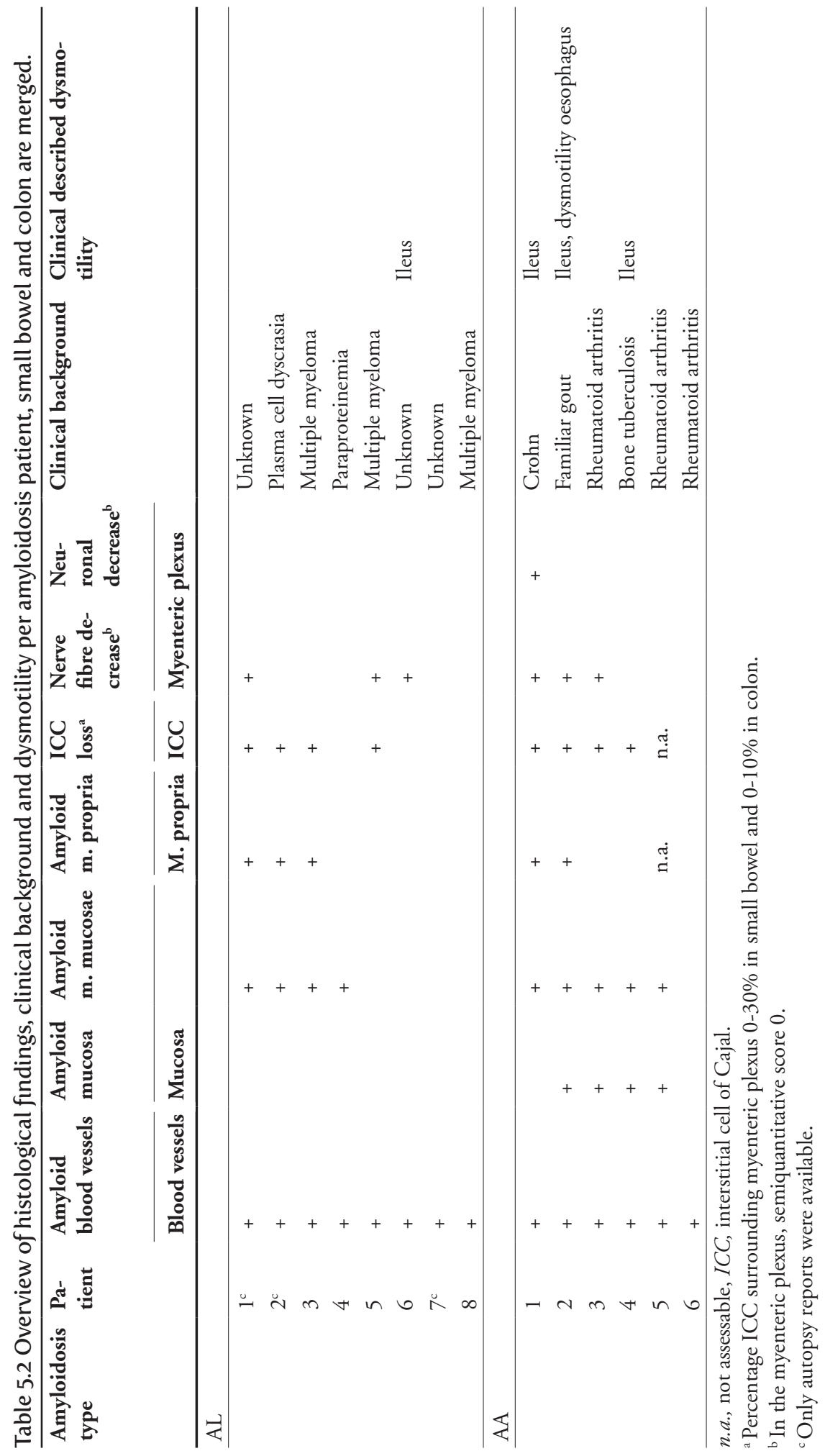


A Localisation of amyloid deposits

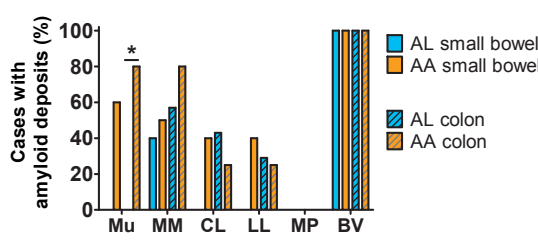

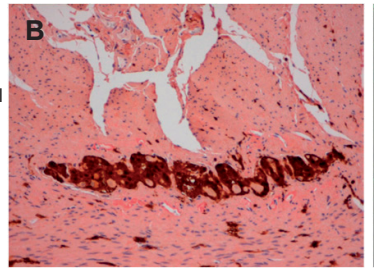

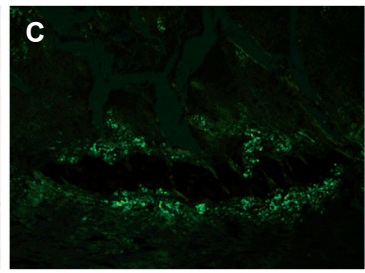

Figure 5.I Amyloid deposition in the bowel wall.

A) Localisation of amyloid deposits in various layers of the intestinal wall. AL compared to AA amyloidosis, in small bowel and colon tissues. B-C) Amyloid in the smooth muscle layers surrounding the myenteric ganglion in an AA patient, double staining S100 (brown) and Congo red (clear red in (B) and apple green in (C). ${ }^{*} \mathrm{p}<0.05$ vs control. $M u$, mucosa, $M M$, muscularis mucosae, $C L$, circular layer, $L L$, longitudinal layer, $M P$, myenteric plexus, $B V$, blood vessel walls.

\section{Bowel musculature}

The presence of amyloid in the musculature of the bowel wall did not result in aberrant staining patterns of $\alpha$-SMA in small bowel or colon (Figure 5.2), which would suggest that no significant $\alpha$-actin filament abnormalities were present in these patients. Polyglucosan inclusion bodies, as a sign of myopathy, were present in the small bowel ( $\mathrm{n}$ $=3)$ and colon $(\mathrm{n}=1)$ of patients with amyloidosis, but there was no consistent relation with the presence of muscular amyloid depositions (Figure 5.2). Some control patients also presented with polyglucosan bodies in small bowel $(\mathrm{n}=3)$ and colon $(\mathrm{n}=1)$. There was no relation between the presence of fibrosis and amyloid (Figure 5.2).

\section{Enteric nervous system}

No amyloid deposits were found inside the myenteric plexus, in some cases amyloid deposits were present in the smooth muscle tissue around this plexus (Figure 5.1). The presence of neurons relative to nerve fibres was evaluated by $\mathrm{HuC} / \mathrm{D}$ staining, the nerve fibre density was assessed by $S 100$ staining. In both the small bowel and colon, no differences were found between the control, AL and AA groups in the submucosal and myenteric plexus as well as in the muscularis propria (Figure 5.3A).

\section{Myenteric ICC network}

Lower percentages of the CD117 positive ICC network surrounding the myenteric plexus were found in the amyloidosis groups compared to the control group, both in the small bowel and colon (Figure 5.3B-C). In the colon, the density of the ICC network was significantly lower in the AL group compared to controls $(\mathrm{p}=0.019)$. Patients with amyloid deposits in the muscularis propria seemed to have a reduced ICC network (red points in Figure 5.3B). In the small bowel of the AL group, no amyloid in the muscularis propria was present, while in two cases of the AA group amyloid was found in the muscularis propria of the small bowel (percentage ICCs around the myenteric 
plexus $0 \%$ and $30 \%$, red points in Figure 5.3B). In the colon, amyloid deposits in the muscularis propria were found in three AL patients (percentage ICCs $0 \%$ ), and in one AA patient (percentage ICCs 10\%).

\section{Assocations in the muscularis propria}

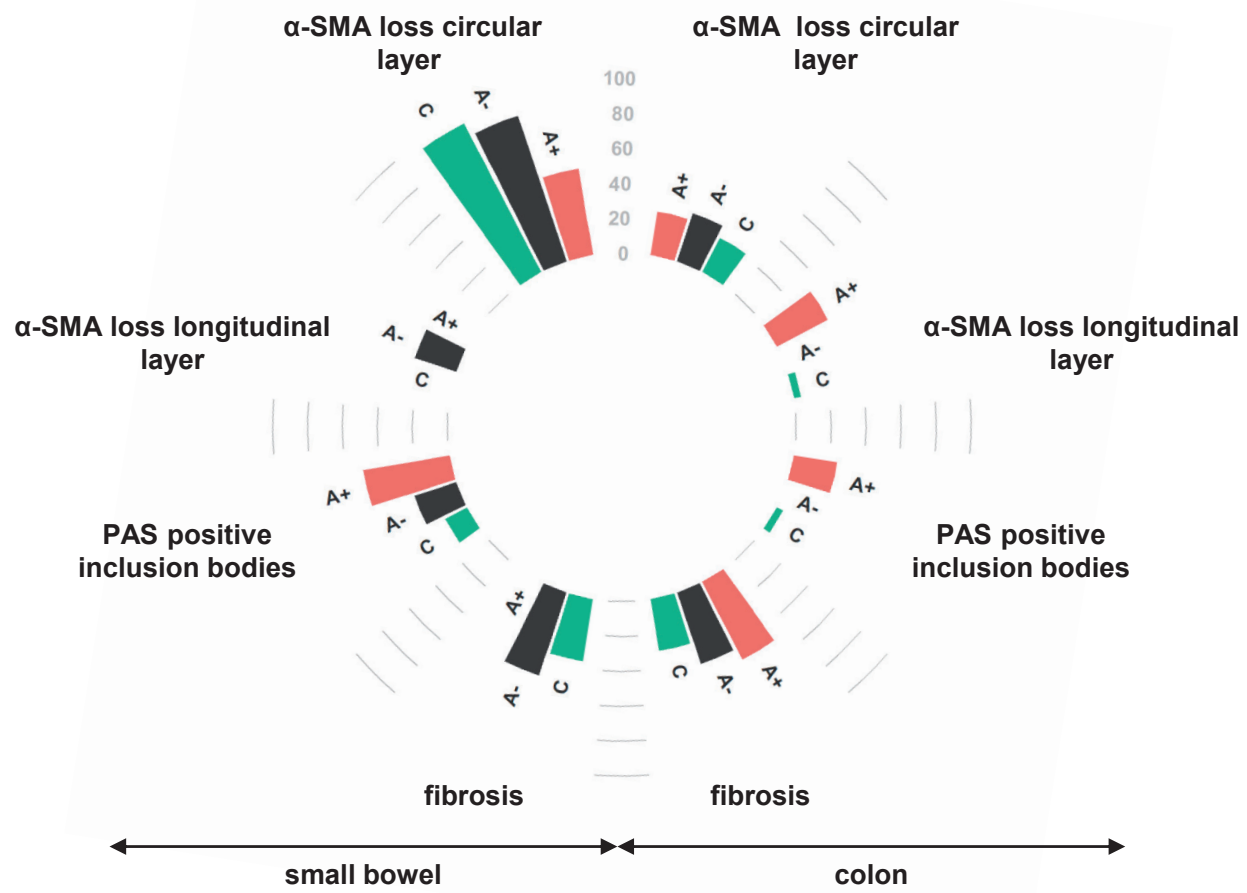

Figure 5.2 Circular plot of aberrations in the musculature of the bowel wall in association with amyloidosis in small bowel (left side) and colon (right side).

Amyloidosis patients were divided according to the presence of amyloid deposits in the musculature $(A+$ : present, $A$-: absent). Percentage of cases are given. $C$, control, $P A S$, periodic acid Schiff staining, $S M A$, smooth muscle actin. 
A
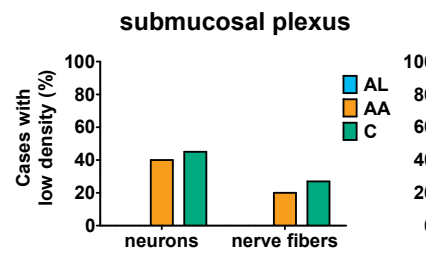

myenteric plexus
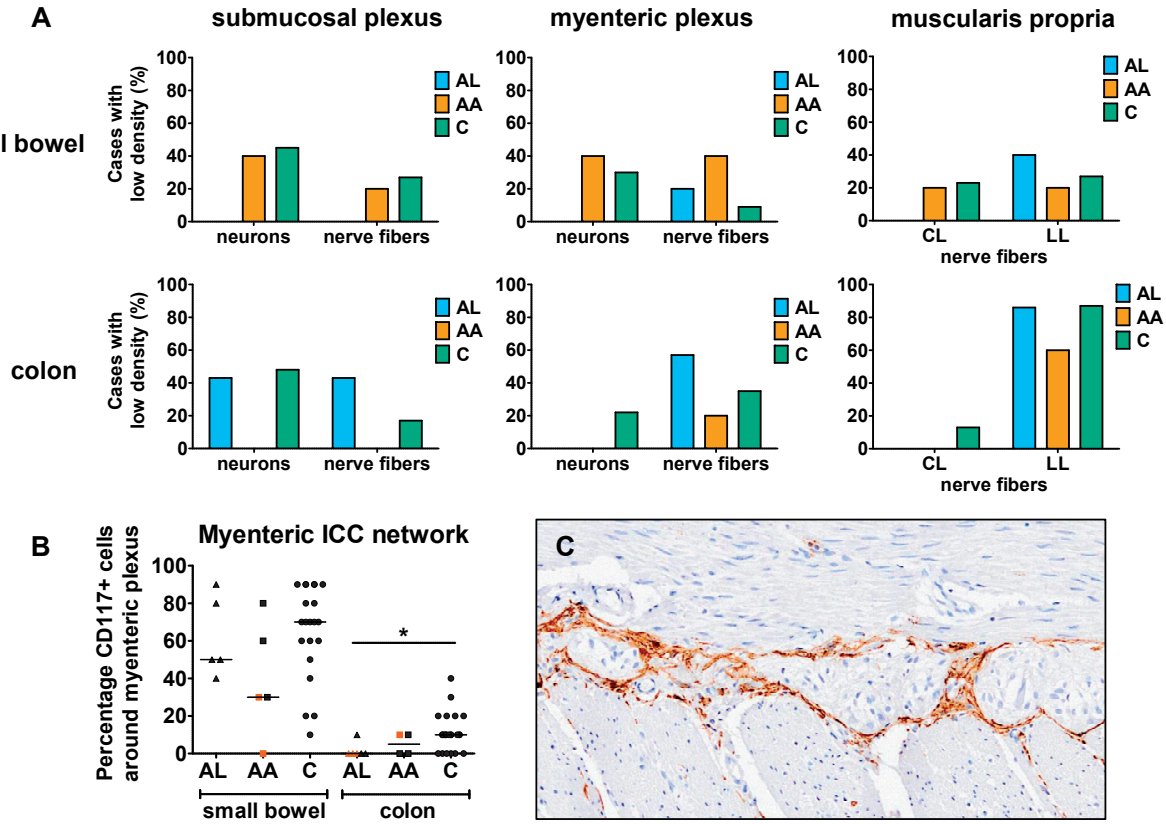

Figure 5.3 Histological findings in the enteric nervous system and the ICC network.

A) The density of neurons and nerve fibres was estimated by $\mathrm{HuC} / \mathrm{D}$ and S100 immunohistochemical staining, respectively. Results for small bowel and colon showed no differences between the AL, AA and control groups. B) Percentage of CD117 positive cells (ICCs) around the myenteric plexus. The black points represent cases without amyloid in the muscularis propria, the red points cases with amyloid in muscularis propria. Horizontal lines show the medians. C) Picture of the positive stained ICC network around the myenteric plexus shows control tissue of small bowel. The percentage in this picture was scored as $100 \%$. ${ }^{*} \mathrm{p}<0.05$ vs control. $A L$, AL amyloidosis, $A A$, AA amyloidosis, $C$, control group, $C L$, circular layer of muscularis propria, $L L$, longitudinal layer of muscularis propria, ICC, interstitial cells of Cajal.

\section{Discussion}

The objective of this study was to investigate the mechanisms that cause intestinal dysmotility in amyloidosis patients, by analysing histological features in a systematic way. Histological findings for individual amyloidosis patients are summarised in Table 5.2. Amyloid deposits were present in the vascular walls of all amyloidosis patients, regardless of type, most prominent in the submucosa. This may contribute to intestinal dysmotility by causing ischaemia and increased permeability. ${ }^{[14]}$ Gastrointestinal bleeding might be caused by disruption of blood vessel walls as a result of amyloid deposition ${ }^{[15,16]}$, which was reported in four of our patients. 
Endoscopic examination of amyloidosis patients frequently show a granular and friable appearance of the mucosa ${ }^{[8,17,18]}$, which can be correlated with amyloid deposits, as was present in our study in $67 \%$ of AA patients. Involvement of the mucosa can lead to diarrhoea and malabsorption. ${ }^{[15,17]}$ It should thus be noted that endoscopic biopsies taken to determine the presence of amyloid, should also include submucosal tissue. When only the mucosa is examined, particularly in AL cases, the results are unreliable. Muscular involvement was present in $64 \%$ of amyloidosis patients, with deposits in all muscular layers, again most pronounced in AA patients. The presence of amyloid deposits in the muscularis propria tended to be associated with the presence of polyglucosan inclusion bodies, which may be related to symptoms of intestinal dysmotility and constipation ${ }^{[19]}$, although this could not be confirmed in our study. The presence of amyloid deposits was not related to either abnormal $\alpha$-actin patterns or fibrosis, suggesting that amyloid induced muscular failure is not because of primary myopathy, but more likely due to mechanical failure.

Histological signs of direct neuropathy could not be found in this study. No amyloid was found inside the myenteric plexus. The presence of amyloid in the smooth muscle surrounding the plexus, which has been suggested to cause secondary degeneration or loss of neurons $s^{[5]}$, was not associated with decreased neuronal or nerve fibre density in our study. Based on our observations, we reject the hypothesis that that intestinal pseudo-obstruction is caused by myopathy in AL amyloidosis and neuropathy in AA amyloidosis. ${ }^{[5,20]}$ This hypothesis is based on the study of Tada et al, where all patients were on total parenteral nutrition because of severe intestinal pseudo-obstruction. ${ }^{[5]}$ These patients are very likely in a late stage of amyloidosis induced motility disorder, where distinction of different mechanisms might be hard. In our study, only one AA patient received total parenteral nutrition, which was given because of dysmotility of the oesophagus. This patient showed amyloid deposits in the mucosa and muscle layers of the intestines. Although no amyloid was found in the myenteric plexus, the ICC network and nerve fibre network were reduced.

We propose an alternative hypothesis about intestinal dysmotility in patients with amyloidosis (Figure 5.4). First, amyloid deposition occurs in the vascular walls, this was shown in all our amyloidosis patients and is commonly found in literature. $[14,21,22]$ Second, amyloid may accumulate in the mucosa and muscular layers of the bowel, decreasing motility by infiltration the smooth muscle tissue. ${ }^{[23-25]}$ Third, involvement of the muscular layers may result in loss of the myenteric ICC network, which may lead to dysmotility. ${ }^{[2]}$ A study into transthyretin amyloidosis patients (i.e. a third form of amyloidosis, non-AA, non-AL) reported loss of the ICC network in the muscularis propria of the stomach without degeneration of the enteric nervous system (ENS). ${ }^{[27]}$ By our knowledge, this is the only study into the ICC network in amyloidosis patients. Neither degeneration nor loss of the ENS was found in our study, suggesting that loss of ICCs may precede damage of the ENS in amyloidosis patients. 


\section{Chapter 5}

Therefore, neurodegeneration and neuronal loss may occur in the final stage of intestinal dysmotility, although this might well be a secondary phenomenon. This sequence would explain intestinal dysmotility in both AL and AA amyloidosis, but there are obvious differences between the subtypes. In AL amyloidosis, endoscopic findings seem to be related to muscular involvement resulting in constipation and intestinal pseudoobstruction, while in AA amyloidosis a fine granular appearance and friability of the mucosa might be related to diarrhoea and malabsorption (Table 5.3).

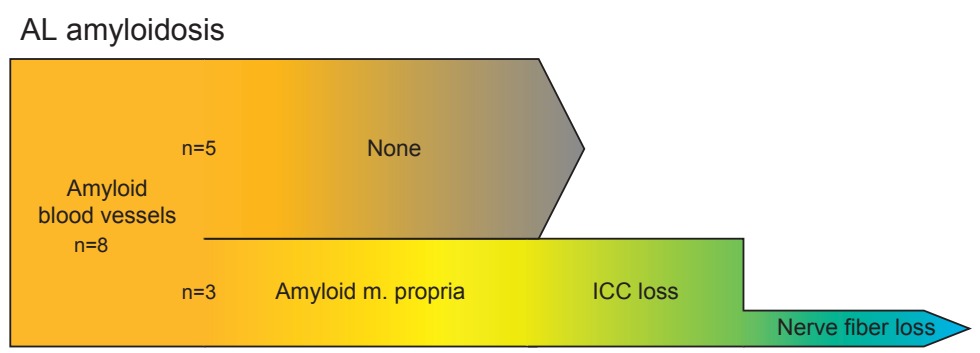

AA amyloidosis

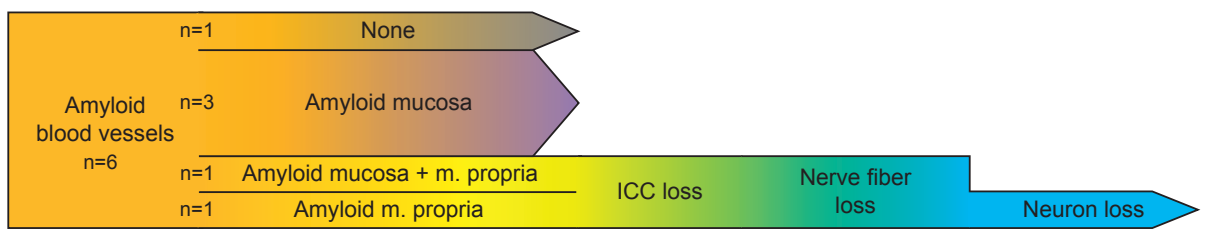

Figure 5.4 Hypothesis about the sequential events that cause intestinal dysmotility in amyloidosis patients.

The process may start with amyloid deposition in the blood vessel walls, which was found in all AL and AA amyloidosis patients, followed by mechanical disruption of the smooth muscles, loss of ICC, and potential involvement of the myenteric plexus (reflected in nerve fibre or neuronal loss). Amyloid deposits were found in the mucosa of AA patients, which may cause symptoms of diarrhoea and malabsorption, but is not directly related to bowel dysmotility. 'None' represents cases without amyloid in both mucosa and muscularis propria. ICC, interstitial cells of Cajal.

Table 5.3 Comparison of AL and AA amyloidosis. ${ }^{[4,8,17]}$

\begin{tabular}{|c|c|c|}
\hline & AL & AA \\
\hline Precursor protein & Immunoglobulin light-chain & Serum amyloid A \\
\hline Organs involved & $\begin{array}{l}\text { Kidneys, heart, digestive system, } \\
\text { nervous system }\end{array}$ & $\begin{array}{l}\text { Kidneys, gastrointestinal tract, liver, heart } \\
\text { (rare) }\end{array}$ \\
\hline $\begin{array}{l}\text { Clinical } \\
\text { presentation }\end{array}$ & $\begin{array}{l}\text { Constipation, mechanical } \\
\text { obstruction, chronic intestinal } \\
\text { pseudo-obstruction, bleeding }\end{array}$ & $\begin{array}{l}\text { Diarrhoea, malabsorption, weight loss, } \\
\text { bleeding }\end{array}$ \\
\hline $\begin{array}{l}\text { Endoscopic } \\
\text { findings }\end{array}$ & $\begin{array}{l}\text { Polypoid protrusions and thickening } \\
\text { of the folds }\end{array}$ & $\begin{array}{l}\text { Fine granular appearance and mucosal } \\
\text { friability }\end{array}$ \\
\hline
\end{tabular}


Our study had several limitations. The study was performed on autopsy material. Hence, the quality of the material was not optimal in some cases, for instance as a result of autolysis of the mucosal layer or imperfect embedding. Therefore, only tissues with all layers of the bowel wall present were included. Scoring of clinical dysmotility was difficult due to limited information in the medical dossier about symptoms related to intestinal dysmotility. Severe dysmotility was described in one AA patient (dysmotility oesophagus, ileus) and may be related to extensive amyloid deposition, ICC loss and reduction of nerve fibres. Loss of nerve fibres in the myenteric plexus may have contributed to ileus in three other patients, although in two of these the ileus could have been a result from earlier bowel resections. The available endoscopic information was insufficient to conclude anything about the relation between microscopic and macroscopic changes. Our study was aimed on exploration of sequential events and forms the basis for future research: larger patients groups and more tissue per patients are required. In those studies, histological examination should be combined with physiological and endoscopic data and accurate knowledge of clinical symptoms of intestinal dysmotility. In this way, the proposed sequential pathogenesis of intestinal involvement in amyloidosis can be investigated more precisely. The AL type of amyloidosis could not be confirmed in three patients in the AL group, but these patients were non-AA (negative amyloid A staining) and non-hereditary transthyretin amyloidosis (Dutch study population ${ }^{[28]}$ ). Excluding these three cases from analysis did not affect the results. The degree of amyloid deposition in AA amyloidosis may be affected by treatment of the underlying disease (in all but one patient), which could have suppressed the acute phase response resulting in reduction of the production and deposition of serum amyloid A protein.

In conclusion, the main initial histological finding in patients with amyloidosis was deposition of amyloid in the vasculature. The most important sign of muscular damage was the presence of amyloid deposits in the musculature, no primary myopathy or neuropathy was detected. We hypothesise that the involvement of the intestines is a sequential process: deposition of amyloid in the vasculature, followed by mechanical disruption of the smooth muscles, loss of ICC and potentially involvement of the myenteric plexus. This final stage may be accompanied by clinical symptoms of severe intestinal dysmotility. 


\section{References}

1. Pettersson T, Konttinen YT. Amyloidosis-recent developments. Semin Arthritis Rheum, 2010. 39(5): p. 356-368.

2. Sipe JD, Benson MD, Buxbaum JN, et al. Amyloid fibril proteins and amyloidosis: chemical identification and clinical classification International Society of Amyloidosis 2016 Nomenclature Guidelines. Amyloid, 2016. 23(4): p. 209-213.

3. Gilat T, Spiro HM. Amyloidosis and the gut. Am J Dig Dis, 1968. 13(7): p. 619-633.

4. Iida T, Yamano H, Nakase H. Systemic amyloidosis with gastrointestinal involvement: Diagnosis from endoscopic and histological views. J Gastroenterol Hepatol, 2018. 33(3): p. 583-590.

5. Tada S, Iida M, Yao T, et al. Intestinal pseudo-obstruction in patients with amyloidosis: clinicopathologic differences between chemical types of amyloid protein. Gut, 1993. 34(10): p. 1412-1417.

6. Tada S, Iida M, Yao T, et al. Endoscopic features in amyloidosis of the small intestine: clinical and morphologic differences between chemical types of amyloid protein. Gastrointest Endosc, 1994. 40(1): p. 45-50.

7. Yamada M, Hatakeyama S, Tsukagoshi H. Gastrointestinal amyloid deposition in AL (primary or myeloma-associated) and AA (secondary) amyloidosis: diagnostic value of gastric biopsy. Hum Pathol, 1985. 16(12): p. 1206-1211.

8. Bansal R, Syed U, Walfish J, et al. Small Bowel Amyloidosis. Curr Gastroenterol Rep, 2018. 20(3): 11.

9. Knowles CH, De Giorgio R, Kapur RP, et al. Gastrointestinal neuromuscular pathology: guidelines for histological techniques and reporting on behalf of the Gastro 2009 International Working Group. Acta Neuropathol, 2009. 118(2): p. 271-301.

10. Petre S, Shah IA, Gilani N. Review article: gastrointestinal amyloidosis - clinical features, diagnosis and therapy. Aliment Pharmacol Ther, 2008. 27(11): p. 1006-1016.

11. Code of Conduct of the Dutch federation of Medical Scientific Societies. [Internet]. Available from: http://www.federa.org. Accessed 8 Dec 2014.

12. den Braber-Ymker M, Lammens M, van Putten MJ, et al. The enteric nervous system and the musculature of the colon are altered in patients with spina bifida and spinal cord injury. Virchows Arch, 2017. 470(2): p. 175-184.

13. den Braber-Ymker M, Heijker S, Lammens M, et al. Practical and reproducible estimation of myenteric interstitial cells of Cajal in the bowel for diagnostic purposes. Neurogastroenterol Motil, 2016. 28(8): p. 1261-1267.

14. Ebert EC, Nagar M. Gastrointestinal manifestations of amyloidosis. Am J Gastroenterol, 2008. 103(3): p. 776-787.

15. Gould M, Zarrin-Khameh N, Sellin J. Small bowel amyloidosis. Curr Gastroenterol Rep, 2013. 15(10): 350 .

16. Chang SS, Lu CL, Tsay SH, et al. Amyloidosis-induced gastrointestinal bleeding in a patient with multiple myeloma. J Clin Gastroenterol, 2001. 32(2): p. 161-163. 
17. Hokama A, Kishimoto K, Nakamoto M, et al. Endoscopic and histopathological features of gastrointestinal amyloidosis. World J Gastrointest Endosc, 2011. 3(8): p. 157-161.

18. Miyaoka M, Matsui T, Hisabe T, et al. Clinical and endoscopic features of amyloidosis secondary to Crohn's disease: diagnostic value of duodenal observation and biopsy. Dig Endosc, 2011. 23(2): p. 157-165.

19. Bernardini N, Ippolito C, Segnani C, et al. Histopathology in gastrointestinal neuromuscular diseases: methodological and ontological issues. Adv Anat Pathol, 2013. 20(1): p. 17-31.

20. Koppelman RN, Stollman NH, Baigorri F, et al. Acute small bowel pseudo-obstruction due to AL amyloidosis: a case report and literature review. Am J Gastroenterol, 2000. 95(1): p. 294-296.

21. Freudenthaler S, Hegenbart U, Schonland S, et al. Amyloid in biopsies of the gastrointestinal tract-a retrospective observational study on 542 patients. Virchows Arch, 2016. 468(5): p. 569577.

22. Ohashi K, Takagawa R, Hara M. Visceral organ involvement and extracellular matrix changes in $\beta 2$-microglobulin amyloidosis - a comparative study with systemic AA and AL amyloidosis. Virchows Arch, 1997. 430(6): p. 479-487.

23. Bitar KN, Gilmont RR, Raghavan S, et al. Chapter 17 - Cellular Physiology of Gastrointestinal Smooth Muscle A2 - Johnson, Leonard R, in Physiology of the Gastrointestinal Tract (Fifth Edition), Ghishan FK, et al., Editors. 2012, Academic Press: Boston. p. 489-509.

24. Gerson LB. Evaluation of the Small Bowel, An Issue of Gastrointestinal Endoscopy Clinics. 2016, Philadelphia, Pennsylvania: E-book. Elsevier Health Sciences: 2016.

25. Legge DA, Wollaeger EE, Carlson HC. Intestinal pseudo-obstruction in systemic amyloidosis. Gut, 1970. 11(9): p. 764-767.

26. Streutker CJ, Huizinga JD, Driman DK, et al. Interstitial cells of Cajal in health and disease. Part I: normal ICC structure and function with associated motility disorders. Histopathology, 2007. 50(2): p. 176-189.

27. Wixner J, Obayashi K, Ando Y, et al. Loss of gastric interstitial cells of Cajal in patients with hereditary transthyretin amyloidosis. Amyloid, 2013. 20(2): p. 99-106.

28. Wixner J, Suhr OB, Anan I. Management of gastrointestinal complications in hereditary transthyretin amyloidosis: a single-center experience over 40 years. Expert Rev Gastroenterol Hepatol, 2018. 12(1): p. 73-81. 


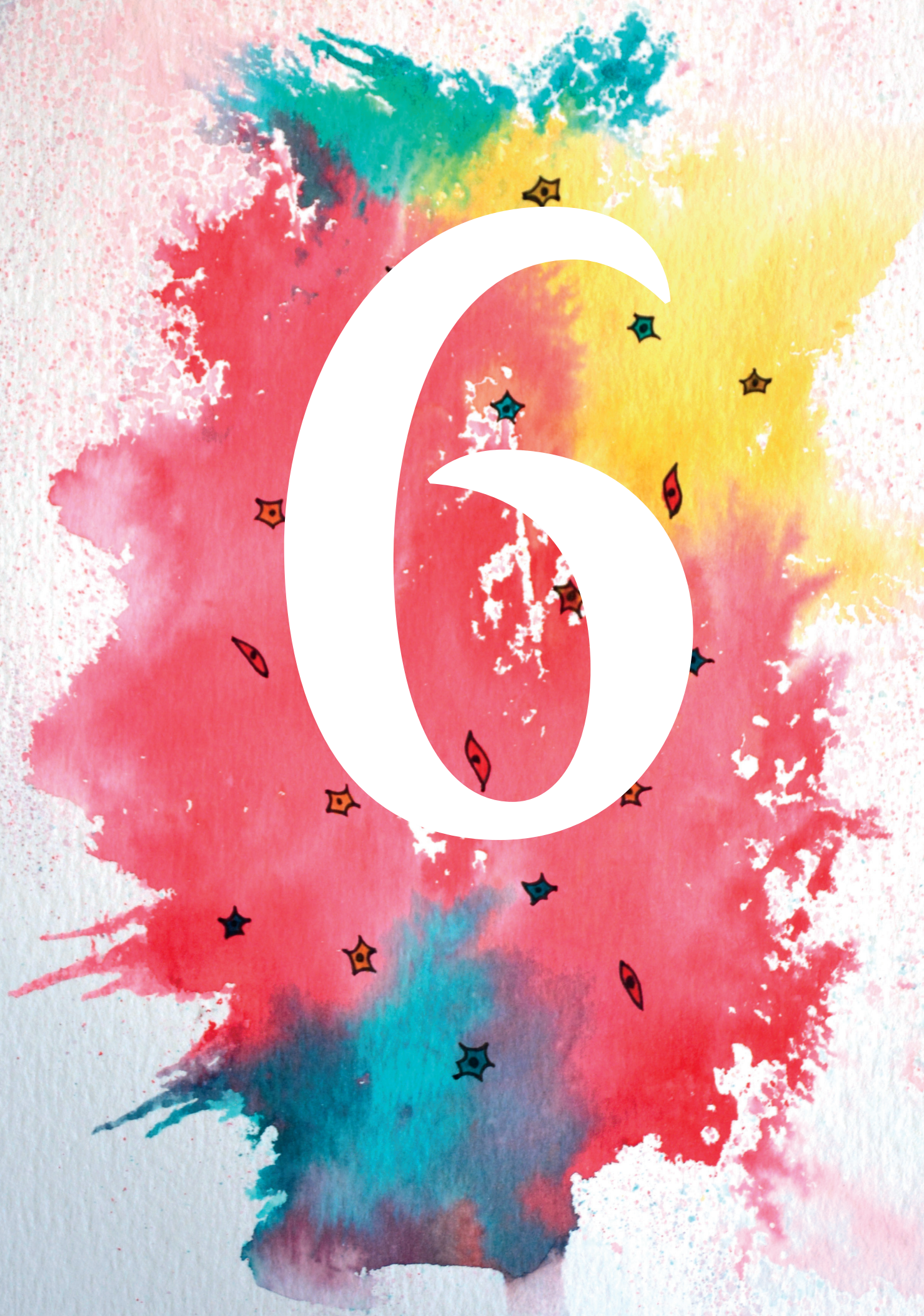




\section{Chapter 6}

\section{Intestinal hypomotility in systemic sclerosis: a histological study into the sequence of events}

M. den Braber-Ymker ${ }^{1}$, M.C. Vonk ${ }^{2}$ K. Grünberg ${ }^{1}$, M. Lammens ${ }^{1,3,4}$, I.D. Nagtegaal ${ }^{1}$

${ }^{1}$ Department of Pathology, Radboud University Medical Center, Nijmegen, The Netherlands

${ }^{2}$ Department of Rheumatology, Radboud University Medical Center, Nijmegen, The Netherlands

${ }^{3}$ Department of Pathology, Antwerp University Hospital, University of Antwerp, Edegem, Belgium

${ }^{4}$ MIPRO, University of Antwerp, Antwerp, Belgium 


\section{Abstract}

The pathogenesis of intestinal involvement in systemic sclerosis (SSc) is thought to be a sequential process (vascular, neuronal, and consecutive muscular impairment), but understanding of the underlying histological changes and how they translate to symptoms, is still lacking. Therefore, we systematically investigated histological characteristics of SSc in the intestines, compared to controls.

Autopsy material from small bowel and colon was used for histological semiquantitative evaluation of the vasculature, enteric nervous system, interstitial cells of Cajal (ICC) and muscle layers, using a combination of histochemical and immunohistochemical stainings, according to guidelines of the Gastro 2009 International Working Group.

Vascular changes were most frequently encountered, represented by intima fibrosis in both arteries and small vessels, and represented by venous dilatation. Second, generalised fibrosis of the circular muscle layer was significantly more found in SSc patients than in controls. Third, reduction of submucosal nerve fibres and myenteric neurons was shown in colon of four SSc patients, which may explain severe symptoms of intestinal dysmotility. The density of myenteric ICC network was decreased in the small bowel of SSc patients.

The postulated sequential processes of intestinal involvement in SSc could not be supported by our histological evaluation. The interpatient diversity suggests that parallel processes occur, explaining the variety of histological features and clinical symptoms. 


\section{Introduction}

Systemic sclerosis (SSc) is an autoimmune disease characterised by inflammation, vasculopathy and fibrosis of the skin and internal organs. Dysfunction of the immune system may result in the production of autoantibodies. ${ }^{[1,2]}$ Although the major causes of death in SSc are pulmonary fibrosis, pulmonary hypertension and cardiac disease, the most common organ complication is involvement of the gastrointestinal (GI) tract. ${ }^{[1,3,}$ ${ }^{4]}$ The oesophagus is most commonly involved (up to $96 \%$ of patients), followed by the small intestine (40-88\%) and colon (10-50\%). ${ }^{[5,6]}$ The development of GI symptoms is diverse, ranging from minimal complaints to significant dysmotility, leading to malnutrition, with wide variation in progression rates. ${ }^{[7-9]}$ Most GI complications originate from decreased motility and have a major effect on the quality of life. ${ }^{[7,8]}$ In the small intestine, the major symptom is hypomotility, sometimes leading to pseudo-obstruction and bacterial overgrowth. ${ }^{[10,11]}$ Manometric studies suggested that neurogenic and myogenic abnormalities may be the underlying cause. ${ }^{[12-14]}$ Cholinergic neurotransmission may be inhibited by the presence of autoantibodies in these patients ${ }^{[15]}$ and deposition of collagen in the small intestinal wall may result in pseudo-obstruction. ${ }^{[11]}$ In the colon, hypomotility can result in delayed transit and constipation. ${ }^{[1]}$

The development of intestinal dysmotility during SSc is currently incompletely understood. It has been hypothesised as a progressive sequence of events, starting with vascular damage. Subsequent neurogenic dysfunction may be the result from ischaemia due to arteriolar changes in the vasa nervorum, compression by collagen deposition around the nerve fibres or blocked cholinergic neurotransmission. ${ }^{[1,16]}$ Finally, the smooth muscle cells become affected, either as a direct result from ischaemia or from neuronal damage. Damaged smooth muscle cells may result in atrophy of the muscle layers and fibrosis. ${ }^{[1,17,18]}$

Systematic analysis of histological changes of the vasculature, neuronal structures and smooth muscle may provide insight in the correlation of pathogenic processes in SSc, from which symptoms may be explained and a possible sequence of events may be deduced. Morphological features in the oesophagus and stomach have been extensively described. ${ }^{[19,20]}$ In the oesophagus, the major finding is smooth muscle atrophy, which may be preceded by loss of neural function, or may be a primary smooth muscle problem. ${ }^{[19]}$ In the stomach, the main findings are fibrosis in the muscularis propria, accompanied by ultrastructural changes of smooth muscle cells and interstitial cells of Cajal (ICCs). ${ }^{[20]}$ In the intestines, no systematic histological study has been performed. Vascular changes have been described in the small arteries and capillaries of the intestines. ${ }^{[18]}$ Some studies reported atrophy and fibrosis in the circular smooth muscle layer of the muscularis propria throughout the GI tract, including small intestine and colon. ${ }^{[16,21]}$ Diffuse fibrosis in the longitudinal muscle layer and the serosa has been reported in the colon as well. ${ }^{[16,18]}$ 
In short, understanding of the underlying histological changes of the proposed sequential processes of intestinal dysmotility is still lacking. Hence, we systematically investigated the histological characteristics of SSc in the small intestine and colon, applying international guidelines for the study on motility disorders. ${ }^{[22]}$ We focused on vasculature, the enteric nervous system (ENS), the myenteric network of ICCs and the muscular layers.

\section{Methods}

\section{Subjects}

Archived autopsy formalin-fixed paraffin-embedded segments of small bowel $(\mathrm{n}=14)$ and colon $(n=18)$ were obtained from SSc patients without selection for dysmotility. Control autopsy material of ileum $(n=22)$ and colon $(n=19)$ was obtained from nonSSc patients without intestinal motility problems and considered as normal.

The local ethics committee approved the study (reference number 2014-1256). Samples were obtained in accordance with the Code of Conduct of the Federation of Medical Scientific Societies in the Netherlands. ${ }^{[23]}$

\section{Tissue preparation}

Sections were cut from formalin-fixed paraffin-embedded full-thickness tissue blocks for conventional histology or immunohistochemistry. Sections were deparaffinised, rehydrated in xylene and ethanol series, and rinsed in tap water by standard protocol.

\section{Histological staining}

Sections of $4 \mu \mathrm{m}$ were used for haematoxylin and eosin (H\&E) and periodic acid Schiff (PAS) staining. Elastic von Gieson (EVG) staining was performed on 6- $\mu$ m sections. Tissues were stained by standard protocols in a Medite TST 30 stainer (Klinipath, Duiven, The Netherlands).

4- $\mu \mathrm{m}$ sections were used for Sirius Red staining. The slides were incubated with a $0.1 \%$ Picro-sirius red solution (Sirius red F3B dissolved in aqueous picric acid) for 1 hour, washed in acetic acid water, dehydrated with ethanol, cleared with xylene and mounted.

\section{Immunohistochemistry}

Immunohistochemical staining was performed on 4- $\mu \mathrm{m}$ sections. Antibodies, suppliers and dilutions are listed in Table 6.1.

For $\mathrm{HuC} / \mathrm{D}$ staining, antigen retrieval was performed in sodium citrate $(\mathrm{pH}$ 6) at $100{ }^{\circ} \mathrm{C}$ for $30 \mathrm{~min}$. Subsequently, endogenous peroxidase was blocked with $3 \%$ hydrogen peroxide in PBS for 20 min. Sections were then rinsed in PBS and incubated 
with primary antibody anti-HuC/D at $4{ }^{\circ} \mathrm{C}$ overnight. After washing in PBS, sections were incubated for $30 \mathrm{~min}$ with a secondary antibody (Powervision poly-HRP anti $\mathrm{Ms} / \mathrm{Rb} / \mathrm{Rt}$ IgG, Immunologic, Duiven, The Netherlands) at room temperature. Subsequently, sections were rinsed in PBS and immunoreactivity was developed with PowerDAB (Immunologic) for $7 \mathrm{~min}$ at room temperature. Sections were finally rinsed in tap water, counterstained with haematoxylin, rinsed in tap water, dehydrated in $100 \%$ ethanol and xylene, and mounted with Permount.

The other immunohistochemical staining reactions were performed in an automated LabVision Autostainer 480 (Klinipath, Duiven, The Netherlands). The method used for antigen retrieval depended on the antibody (Table 6.1). Subsequently, endogenous peroxidase was blocked with 3\% hydrogen peroxide in methanol for $10 \mathrm{~min}$. Sections were incubated with primary antibody for $60 \mathrm{~min}$. Subsequently, the sections were incubated with Powervision poly-HRP anti Ms/Rb/Rt for $30 \mathrm{~min}$, followed by staining with PowerDAB for $7 \mathrm{~min}$ and counterstaining with haematoxylin for $1 \mathrm{~min}$. All incubations were performed at room temperature.

Tissue blocks containing different tissue types were used as controls, with known staining patterns for both positive and negative stained tissues.

Table 6.I Primary antibodies used for immunohistochemistry.

\begin{tabular}{lllll}
\hline Antibody & Clone & Manufacturer & Dilution & Antigen retrieval \\
\hline $\mathrm{HuC} / \mathrm{D}$ & 16A11 & Molecular Probes & $1: 600$ & $\begin{array}{l}\text { Sodium citrate } 10 \mathrm{mM} \\
(\mathrm{pH} 6.0) 30 \text { min at } 100^{\circ} \mathrm{C}\end{array}$ \\
$\begin{array}{l}\text { S100 } \\
\text { CD117 }\end{array}$ & polyclonal & DAKO & $1: 10000$ & EDTA pH 910 min at $96^{\circ} \mathrm{C}$ \\
$\begin{array}{l}\alpha \text {-smooth } \\
\text { muscle actin } \\
(\alpha-S M A)\end{array}$ & 1 A4 & Immunologic & $1: 200$ & None \\
\hline
\end{tabular}

\section{Microscopic analysis}

Sections were evaluated blind to diagnosis. The histology of the bowel wall was examined by H\&E. PAS was used to verify the presence or absence of polyglucosan inclusion bodies in the muscularis propria. The presence or absence of fibrosis was assessed in the submucosa, muscularis propria, and myenteric plexus on EVG stained sections, represented by focal accumulation of collagen in these parts of the bowel wall. Quantification of fibrosis in both layers of the muscularis propria was performed on Sirius Red stained sections using semi-automated image analysis.

The vasculature was evaluated on EVG stained sections. Intima fibrosis in submucosal arteries, veins and small vessels was scored as absent or present. The presence of venous dilatation was analysed and graded as absent, slight, moderate, or severe. 
Immunohistochemically stained sections were assessed by semiquantitative scoring using visual analysis to evaluate systematically the neuronal structures and smooth muscle layers, as previously described. ${ }^{[24]}$ The presence of neurons in ganglia was analysed on $\mathrm{HuC} / \mathrm{D}$-stained sections. The number of neurons in relation to the present plexus was estimated in $\mathrm{HuC} / \mathrm{D}$ sections as follows: 0 (no neurons/low neuronal density) and 1 (high neuronal density). S100 was used to assess the distribution of nerve fibres (including nuclei of glial cells) in the submucosa, the myenteric plexus, and both muscle layers of the muscularis propria. The degree of distribution was scored as follows: 0 (no/low density) and 1 (high density of positive fibres). ${ }^{[24]}$ The network of ICCs surrounding the myenteric plexus was estimated on CD117-stained sections as described earlier. ${ }^{[25]}$ The percentage of the circumference which is covered by CD117positive cells was rated from 0 to $100 \%$ in $10 \%$ increments. Thus, a percentage of $0 \%$ represented no positive cells around the ganglia and in sections estimated as $100 \%$, the ganglia were completely surrounded by CD117-positive cells. $\alpha$-Smooth muscle actin $(\alpha-S M A)$ staining was used to assess the muscular layers. Staining intensities of circular and longitudinal muscle layers were classified in two grades: 0 (no/weak) and 1 (strong staining intensity). ${ }^{[24]}$

\section{Quantification of fibrosis}

Sirius Red stained sections were scanned using a 3DHistech Pannoramic 250 Flash II scanner (3DHistech, Budapest, Hungary). Slides were digitised using a $20 \times$ objective (resultant pixel resolution of 0.24 microns). A maximum of ten images of both the circular and longitudinal muscle layer per patient were taken at 20x magnification using Pannoramic Viewer (3Dhistech), dependent on the size of the tissue fragment. Per image, the muscle area was manually annotated using a bright green coloured line. Staining artefacts and large blood vessels were excluded from the annotations. Subsequently, the images were analysed using a modified version of the ImageJ macro developed by Hadi et al. ${ }^{[26]}$, using Fiji (Fiji is Just ImageJ $1.47 \mathrm{v}^{[27]}$ ). Our macro included the area within the bright green demarcated annotation for analysis, while Hadi et al. excluded the area within the green annotation. Other minor changes in the Fiji macro were made to optimise the analysis for our tissue sections. The batch mode macro was used to analyse multiple images in a row. The modified batch mode macro is available on request. The percentage of fibrosis (collagen) compared to the total amount of tissue within the green demarcated area in an image was quantified for the circular and longitudinal muscle layer, respectively.

\section{Differentiating focal and generalised fibrosis}

The presence of fibrosis on EVG stained sections in circular and/or longitudinal layers was defined as 'focal fibrosis'. The amount of collagen (Sirius Red) in the circular layer was represented as a percentage of fibrosis (Figure 6.3C). We calculated 95\% confidence 
intervals (CI) of means for each group: for SSc, lower CI were 48\% (small bowel) and $47 \%$ (colon), and for controls, upper CI were 43\% (small bowel) and 45\% (colon). Consequently, we defined a percentage of collagen higher than $48 \%$ as 'generalised fibrosis'.

\section{Statistical analysis}

Categorical variables were described by percentages. Differences between categorical variables were assessed by the chi-square test (likelihood ratio, exact p-values compared with the control group). Continuous variables were presented as means \pm standard deviation (SD). The Mann-Whitney test was performed to compare the CD117 scores between groups. Wilcoxon signed-rank and $\mathrm{McNemar}$ tests were used for pairwise comparison of small bowel and colon tissues within one patient. In quantification of fibrosis, $\mathrm{T}$ tests were used to compare SSc and control groups, paired T tests for comparisons within one patient. A p-value of 0.05 was considered significant. Data were analysed by the IBM SPSS Statistics 22 Software (SPSS Inc., Chicago, IL, USA) and GraphPad Prism version 5.00 for Windows (GraphPad Software, San Diego California USA).

\section{Results}

\section{Patients}

Tissue material of 21 patients with SSc and 26 control patients was evaluated in this study. The SSc group consisted of 14 small bowel and 18 colon cases, from 11 patients tissues of both locations were analysed. In the control group, 22 small bowel cases were included, 19 colon cases, and of 15 patients both small bowel and colon tissues were evaluated. The mean age of the SSc group was $58 \pm 12$ years (38\% male); in the control group $66 \pm 14$ years (59\% male). Information about SSc progression was obtained from the autopsy reports and patient dossiers. Twelve patients had the limited cutaneous form of SSc (lcSSc), nine patients the diffuse form of SSc $\left(\mathrm{dcSS}_{\mathrm{c}}\right)$. Four patients reported clinical symptoms of severe gut dysmotility (e.g. pseudo-obstruction or need for total parenteral nutrition), twelve reported oesophageal involvement, seven reported cachexia (not explicitly related to hypomotility). Fourteen patients had proven lung disorder, four patients had proven renal involvement.

\section{Vasculature}

Vascular changes tended to be more often present in patients with SSc compared to controls, as evident by trends present in all analyses. Only intima fibrosis in the submucosal arteries of the small bowel showed a significant difference (50\% versus $9 \%$, $\mathrm{p}=0.014$, Figure 6.1). There were no differences between vascular abnormalities in small bowel and colon in SSc, except for the more frequent presence of intima fibrosis in the small vessels of the small bowel $(p=0.046)$. 

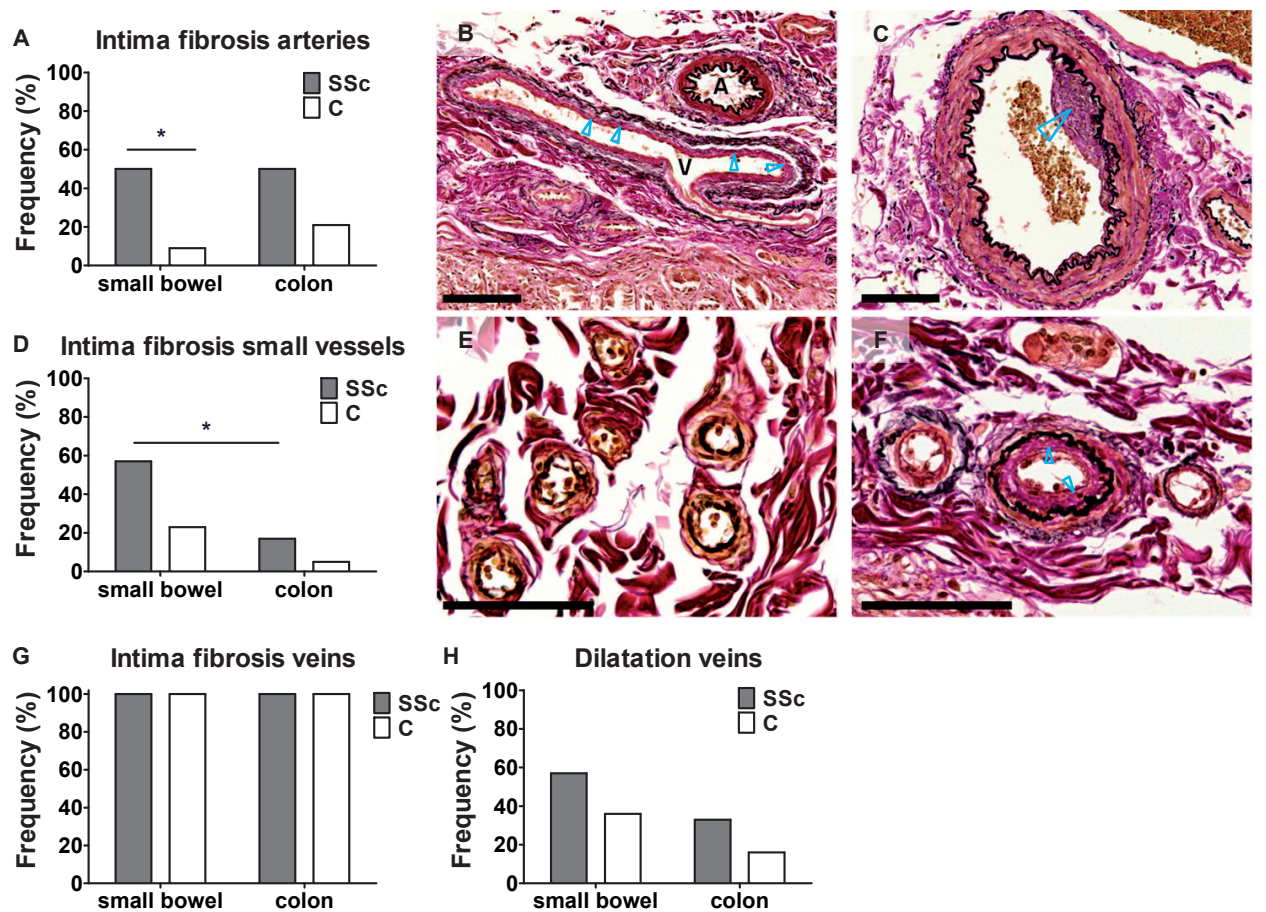

Figure 6.I Histological findings in the vasculature.

A) Significantly more intima fibrosis was found in the submucosal arteries of the small bowel in SSc. B) shows a normal artery $(A)$ and a vein $(V)$ with intima fibrosis (pink band around lumen, blue arrowheads), EVG. C) An artery with intima fibrosis (blue arrowhead), EVG. D) Intima fibrosis in the small vessels was more frequently found in SSc patients compared to controls, not significant. Intima fibrosis was more present in small bowel than in colon. E) Normal small vessels and (F) small vessels with intima fibrosis (pink band above internal elastical lamina, blue arrowheads), EVG. G) Venous intima fibrosis was found in all control and SSc cases, a representative example is given in (B), indicated with ' $V$ '. H) Dilatation of veins was more frequently observed in SSc, not significant. ${ }^{*} \mathrm{p}<0.05$ vs control. Scale bars $100 \mu \mathrm{m}$. C, control, $S S c$, systemic sclerosis, $E V G$, elastic von Gieson.

\section{Enteric nervous system}

To evaluate the enteric nervous system we assessed both neuronal density and nerve fibre density, in particular in the submucosal plexus, the myenteric plexus and the muscularis propria. In the small bowel, no differences were observed between the SSc and the control group for the enteric nervous system (Figure 6.2A-C). In the colon, neuronal density was decreased in the myenteric plexus $(74 \%$ versus $35 \%, p=0.042$, Figure 6.2E) of SSc patients, but not in the submucosal plexus (Figure 6.2D). In contrast, the density of the submucosal nerve fibre network was frequently very low around the submucosal plexus of SSc patients (79\% versus 37\%, p = 0.020, Figure 6.2D), but not in the myenteric plexus (Figure 6.2E), nor in the muscularis propria (Figure 6.2C). 
A Small bowel submucosal plexus

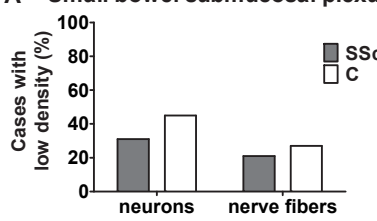

D Colon submucosal plexus
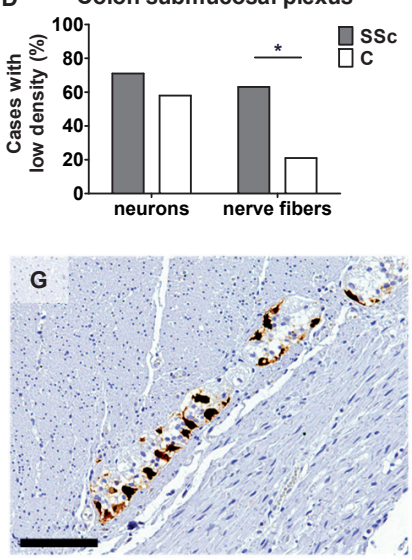

B Small bowel myenteric plexus
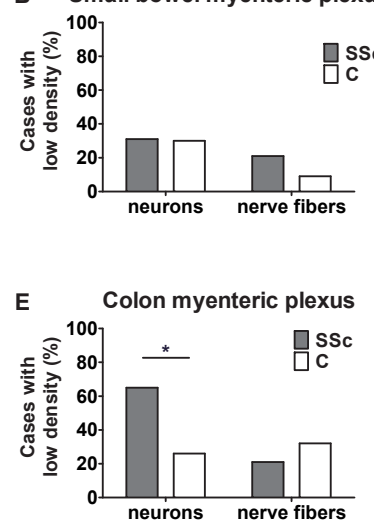

H

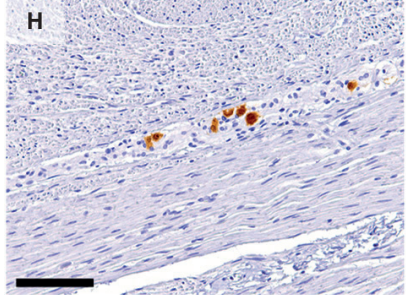

C Nerve fiber density m.propria

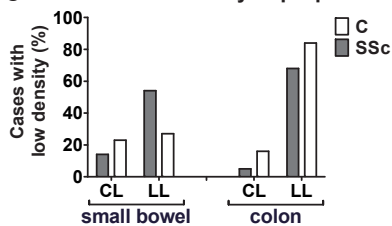

Myenteric ICC network
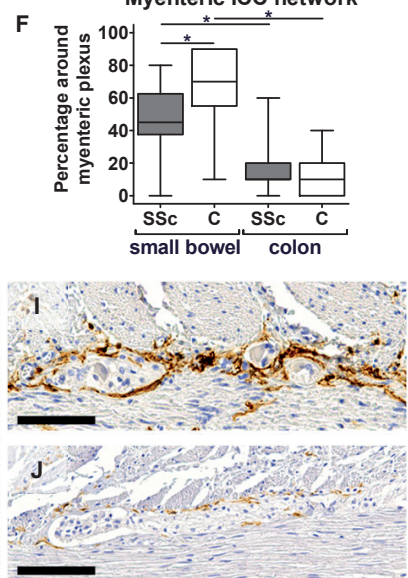

Figure 6.2 Histological findings in the enteric nervous system.

$\mathrm{A}$ and $\mathrm{B}$ ) show the percentage of cases with low density of neurons (HuC/D) and nerve fibres (S100) in the submucosal and myenteric plexus of small bowel, respectively. In (C), the percentage of cases with a low S100-positive nerve fibre density in the muscularis propria (score 0) is shown. D and E) show the percentage of cases with low density of neurons and nerve fibres in the submucosal and myenteric plexus of colon, respectively. In the SSc group, the nerve fibre density is significantly decreased in the submucosal plexus (D) and the neuronal density is decreased in the myenteric plexus of colon (E). F) The ICC network surrounding the myenteric plexus was significantly impaired in the small bowel in SSc. Representative examples of the $\mathrm{HuC} / \mathrm{D}$ positive neurons in the myenteric plexus of a control sample $(\mathrm{G})$ shows a higher neuronal density than that of a SSc section (H). In (I), a myenteric plexus of the small bowel is shown of a control that is completely surrounded by CD117 positive ICCs. J) shows an example of the impaired myenteric ICC network in the small bowel in SSc. ${ }^{*} \mathrm{p}<0.05$. Scale bars $100 \mu \mathrm{m}$. C, control, SSc, systemic sclerosis, $C L$, circular layer, $L L$, longitudinal layer, $I C C$, interstitial cell of Cajal.

\section{Myenteric ICC network}

In the small bowel, significantly less CD117 positive cells were present around the circumference of the myenteric plexus in SSc, compared to the control group (33 $\pm 24 \%$ versus $65 \pm 25 \%, \mathrm{p}=0.024)$. In the colon, the ICC network surrounding the myenteric plexus was comparable in both groups $(15 \pm 16 \%$ versus $12 \pm 11 \%, \mathrm{p}=0.751$, Figure $6.2 \mathrm{~F})$. In both groups, CD117 scores were higher in the small bowel as compared to colon $(\mathrm{p}<0.005)$. 


\section{Muscularis propria}

To evaluate the muscularis propria, we investigated the smooth muscle cells ( $\alpha$-SMA immunohistochemistry), the presence of polyglucosan inclusion bodies, and the presence of fibrosis. No difference was present between SSc patients and controls with respect to the $\alpha$-SMA filament proteins. Overall, more inclusion bodies were found in SSc patients compared to controls ( $25 \%$ versus $7 \%, p=0.05$, Figure $6.3 \mathrm{~A})$. The presence of fibrosis was evaluated in both the circular and longitudinal muscle layer in two ways: presence of focal fibrosis was evaluated on EVG stained sections, while generalised fibrosis was evaluated on Sirius Red stained sections by quantification of the amount of collagen. Focal fibrosis is more frequently present in patients with SSc in particular in the circular layer of the colon ( $26 \%$ versus $0 \%, \mathrm{p}=0.046$, Figure $6.3 \mathrm{~B})$. Generalised fibrosis was also more often present in the circular layer of SSc patients (small bowel: $60 \pm 18 \%(\mathrm{n}=12)$ versus $36 \pm 16 \%(\mathrm{n}=21), \mathrm{p}<0.001$; colon: $61 \pm 25 \%$ $(\mathrm{n}=14)$ versus $37 \pm 18 \%(\mathrm{n}=19), \mathrm{p}=0.003$, Figure $6.3 \mathrm{C})$. No differences between the $\mathrm{SSc}$ and control group were found in the longitudinal layer.
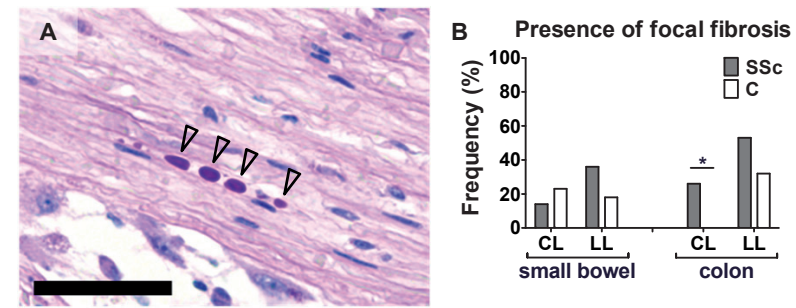

C Generalised fibrosis in the circular layer
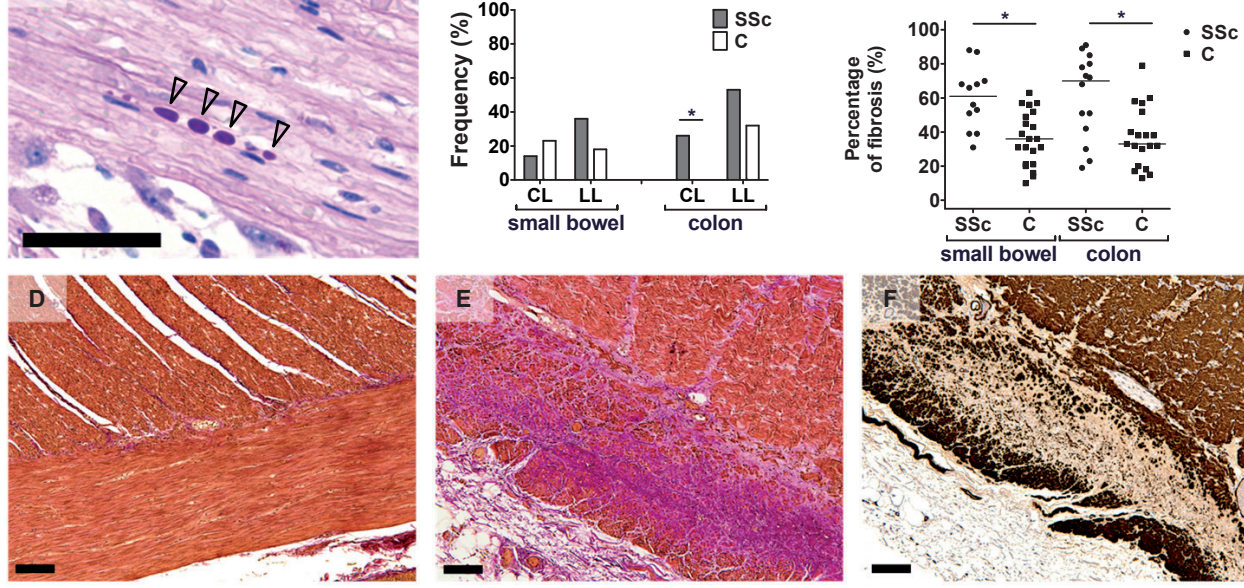

Figure 6.3 Histological findings in the muscularis propria.

Polyglucosan inclusion bodies were more often present in the SSc group. A) shows an example of these inclusion bodies (black arrowheads). Areas with remarkable fibrosis were scored on EVG stained sections (C, $\mathrm{D}, \mathrm{E})$. B) shows the presence of focal fibrosis in the circular layer (CL) and longitudinal layer (LL) of the small bowel and colon, respectively. Fibrosis was significantly more frequently found in the CL of the colon in SSc patients (A). The percentage of generalised fibrosis was computed on Sirius Red stained sections (C). Significantly more fibrosis was found in the circular layer in SSc patients compared to controls in both the small bowel and colon. D) shows the muscularis propria of the colon without fibrosis in a control section, EVG. Excessive collagen accumulation (pink) in the LL of a colon section of SSc reveals fibrosis in (E), EVG. In the same region, the absence of $\alpha$-SMA staining is found (E). ${ }^{*} \mathrm{p}<0.05$. Scale bars $100 \mu \mathrm{m} . C$, control, $S S c$, systemic sclerosis, $E V G$, elastic von Gieson, $S M A$, smooth muscle actin. 


\section{Sequential evaluation of histology in SSc}

Fibrosis of the intestinal muscular layers is considered the end-stage of gastrointestinal SSc (Figure 6.4A). Three subgroups were created: SSc patients with generalised fibrosis in the circular layer, SSc patients with focal fibrosis, and SSc patients with no relevant fibrosis in the muscle. Vascular and neural abnormalities were compared for these groups, but no differences could be observed (Figure 6.4B).

In the small bowel, all SSc patients with and without fibrosis showed some form of vascular changes (i.e. intima fibrosis in arteries, in small vessels and/or venous dilatation). In the colon, no vascular changes were observed in 3 of 10 patients with generalised fibrosis and in 1 of 2 patients without fibrosis. Vascular changes appeared to be more present in small bowel than in colon. Changes in the ENS tended to be more frequently present in the colon than in the small intestine. In the colon, 4 of 7 patients with decreased neurons in both plexuses and a reduced nerve fibre network in the submucosal plexus reported symptoms of severe intestinal dysmotility. This association was not found in the small intestine. Patients with a low percentage of ICCs around the myenteric plexus did not present with symptoms of gut dysmotility. No differences between the lcSSc and dcSSc types were observed (Figure 6.4B). 


\section{Chapter 6}

A Proposed sequential process of intestinal involvement in SSc

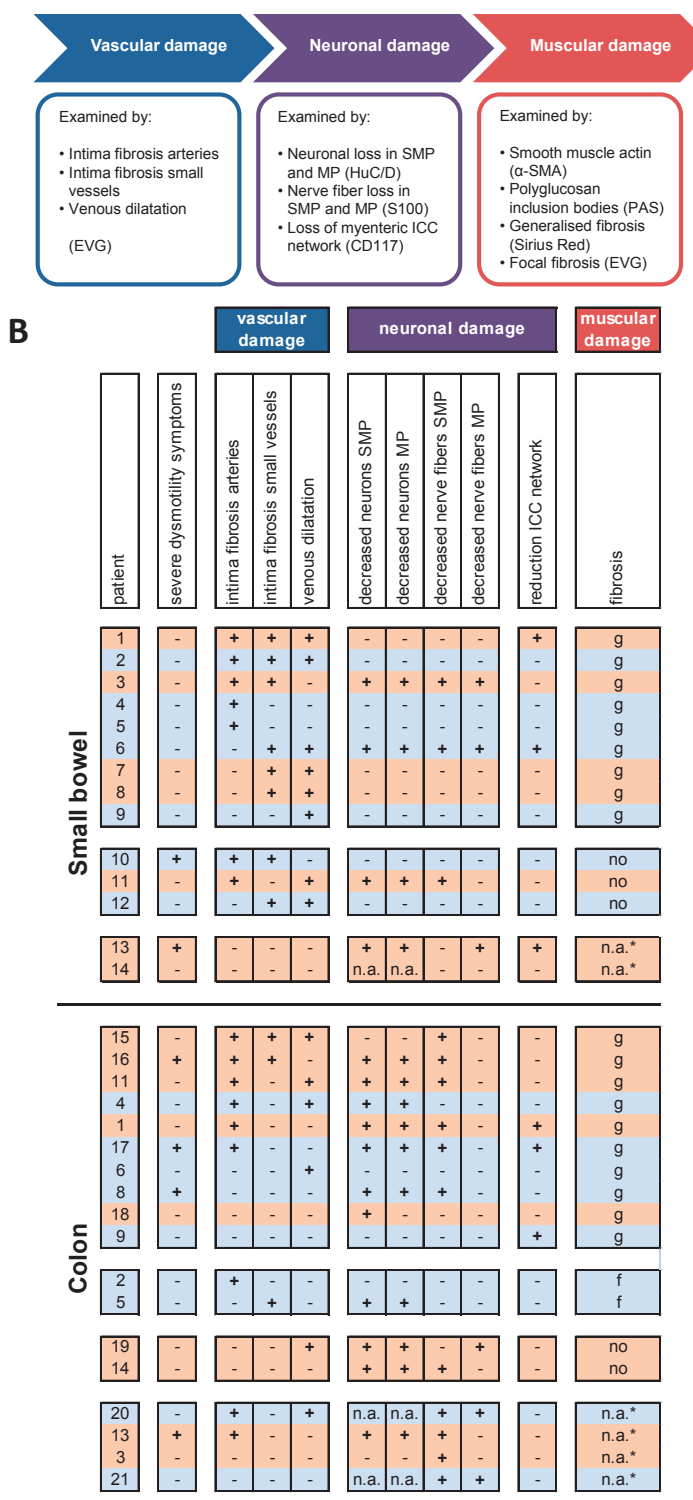

Figure 6.4 Evaluation of the proposed sequential pathogenesis of intestinal involvement in SSc.

Vascular, neuronal and muscular damage were examined by several parameters (A). In (B), an overview is given of each SSc patient, set against the presence of severe dysmotility symptoms, forms of vascular damage, neuronal damage and muscular damage. The proposed end-stage of the disease is fibrosis of the muscularis propria. Three subgroups were distinguished: generalised fibrosis in the circular muscle layer $(g)$, focal fibrosis $(f)$, and no fibrosis $(n o)$. Each patient number correlates with one individual SSc patient. Orange colour represents patients with limited cutaneous SSc, blue colour patients with diffuse cutaneous SSc.

${ }^{*}$ no focal fibrosis was present, but generalised fibrosis was not assessable. MP, myenteric plexus, SMP, submucosal plexus, ICC, interstitial cell of Cajal, n.a., not assessable. 


\section{Discussion}

The histological features of SSc in the intestines were systematically investigated, focusing on vasculature, ENS, myenteric network of ICCs and smooth muscle layers of the gut wall. The most frequent finding was vascular change in SSc patients in both small bowel and colon, represented by intima fibrosis in the arteries and small vessels (particularly pronounced in small bowel), and venous dilatation. Vascular intima fibrosis has been described in other organs affected by SSc, including the oesophagus and lungs. ${ }^{[19,28]}$

Second, remarkable generalised fibrosis was present in the circular layer of the small bowel and colon of SSc patients. SSc has been shown to commonly involve the circular muscle layer. ${ }^{[16,21]}$ Our findings confirm this and extend it by showing focal fibrosis being more frequently present in the longitudinal layers of small bowel and colon of SSc patients. In the oesophagus, Roberts et al assessed the loss of muscle cells (defined as 'smooth muscle atrophy') and they defined thickening of the muscle layer as 'fibrosis'. ${ }^{[19]}$ The figures in their study show replacement of smooth muscle cells by collagen. We found collagen patterns similar to that in the oesophagus, defined here as 'fibrosis'. Polyglucosan inclusion bodies were significantly more frequently observed in SSc cases, which may be associated with intestinal myopathy. ${ }^{[29]}$ There was no clear relationship between the presence of polyglucosan inclusions and symptoms of intestinal dysmotility in our study.

Third, we did not find changes of the ENS in the small bowel of SSc patients, but some significant differences between SSc patients and controls were shown in colon: less nerve fibres were found in the submucosal plexus, and the neuronal density in the myenteric plexus was decreased. This may be related to symptoms of intestinal hypomotility. In addition, the ICC network around the myenteric plexus in the small bowel of SSc patients was reduced compared to the control group, which was not clearly associated with symptoms of dysmotility. Loss of ICCs can be observed as secondary to the absence of enteric neurons, for example in Hirschsprung's disease ${ }^{[30,31]}$ or to impaired extrinsic innervations, as is the case in spinal cord injury ${ }^{[24]}$. However, we did not find any correlation between decreased neuronal density and myenteric ICC scores in our study.

Comparison of small bowel and colon samples from each individual patient, showed that intima fibrosis in the small vessels was more frequent in the small bowel, as was the presence of polyglucosan inclusion bodies. Clinically, up to $90 \%$ of SSc patients have complaints from involvement of the small bowel including hypomotility, secondary bacterial overgrowth and small bowel pseudo-obstruction. ${ }^{[11]}$ An association between histological changes and these severe small intestinal complaints could not be found in our study. In the colon, more changes in the ENS were found compared to small bowel. Furthermore, focal fibrosis of the muscularis propria was more pronounced in the colon than in the small bowel. However, the presence of generalised fibrosis in 
the circular muscle layer may have more effect on smooth muscle function than focal fibrosis. Reduction of the ENS may explain the symptoms of severe colonic dysmotility in four patients. Clinically, colon involvement is less frequent, and consists of delayed transit due to hypomotility. ${ }^{[11]}$

Intestinal involvement in SSc has been proposed as a sequential process, which might start with vascular damage, followed by autonomic neuronal dysfunction, smooth muscle atrophy and fibrosis. ${ }^{[17,18,32]}$ However, the exact mechanisms are still poorly understood. ${ }^{[1]}$ Since fibrosis of the muscularis propria is the considered end-stage lesion, we expected that in patients with fibrosis changes in the vasculature and ENS would be present as well (because we assume that these changes are not reversible). Case studies have indeed indicated that neuronal damage precedes smooth muscle atrophy and fibrosis in the gastrointestinal tract. ${ }^{[16,18]}$ However, in our larger series we could find histological indications for this correlation only in part of SSc patients: vascular and neuronal changes were shown in small bowel in two of nine patients and in colon in six of ten patients with generalised fibrosis of the muscularis propria. Therefore, the pathogenic mechanisms of gut involvement may be more complex. Our results indicate that parallel processes could be involved in part of SSc patients rather than sequential processes. The initial event in the pathogenesis of intestinal SSc may be either autonomic axonal degeneration (sympathetic overactivity) resulting in vascular instability, or autoimmune related (e.g. antibodies against myenteric neurons), or a combination of both. ${ }^{[33]}$ Manometric studies have shown evidence for a neuropathic phase preceding a myopathic phase in gut involvement. ${ }^{[34,35]}$ However, SSc patients may develop manometric abnormalities before the presence of histological changes ${ }^{[1]}$, which may explain that we did not find histological neuropathic features in all patients with generalised fibrosis of the circular muscle layer. Results of manometry were not available in our study. On the other hand, smooth muscle function may directly be affected by different processes: tissue ischaemia as result of vascular instability may trigger a repair reaction, leading to excessive collagen deposition. ${ }^{[33]}$ Furthermore, antibodies against the muscarinic-3 receptor have been found in SSc and may play a critical role in dysmotility in blocking the cholinergic neurotransmission and inhibition of acetylcholine action at smooth muscle cells. ${ }^{[15,36,37]}$ In addition, altered TGF- $\beta$ signalling may be related to spontaneous fibrosis and subsequent dysmotility in mouse colon. ${ }^{[38]}$

A limitation of this study was the quality of the autopsy material. The mucosa was not assessable due to autolysis, but the other structures of the gut wall were still present. Some tissue sections could only partly be analysed, because of improper tissue orientation. Limited tissue samples were used, which could have been resulted in missed histological abnormalities elsewhere in the intestines. Although we studied a large patient group for a rare disease as SSc, for statistical analysis the groups were small. Nevertheless, this is the first systematic study in the small bowel and colon using international guidelines for histological evaluation of the bowel wall, providing new 
insights in the complexity of the disease. The use of antibodies for (semi)quantification allows for more complete histological evaluation of neuronal damage. Neurons and nerve fibres were studied with $\mathrm{HuC} / \mathrm{D}$ and S100, respectively, resulting in the first data using this antibodies for SSc. Relevant information about clinical symptoms of intestinal hypomotility may have been missed, because of the retrospective character of our study. Currently, more clinical data are reported in the patient dossiers than for example twenty years ago, which could have caused underestimation of intestinal symptoms. Future studies should include tissues of SSc patients with known intestinal dysmotility, to combine histological features with clinical symptoms. In addition, research into the presence of autoantibodies in the (human) ENS and smooth muscle may provide new insights into involvement of these important structures for gut motility. Use of bowel resection material instead of autopsy material is recommended to improve tissue quality.

In conclusion, the postulated sequential processes leading to intestinal dysmotility in SSc are vascular damage, neuronal dysfunction, smooth muscle atrophy and fibrosis. This sequence could not be supported by our histological findings. The histological variation between SSc patients may suggest that pathological processes are parallel rather than sequential, explaining the diversity of histological features and clinical symptoms. 


\section{References}

1. Kumar S, Singh J, Rattan S, et al. Review article: pathogenesis and clinical manifestations of gastrointestinal involvement in systemic sclerosis. Aliment Pharmacol Ther, 2017. 45(7): p. 883898.

2. Katsumoto TR, Whitfield ML, Connolly MK. The pathogenesis of systemic sclerosis. Annu Rev Pathol, 2011. 6(1): p. 509-537.

3. Pellar RE, Pope JE. Evidence-based management of systemic sclerosis: Navigating recommendations and guidelines. Semin Arthritis Rheum, 2017. 46(6): p. 767-774.

4. Ebert EC. Gastric and enteric involvement in progressive systemic sclerosis. J Clin Gastroenterol, 2008. 42(1): p. 5-12.

5. Tiev KP, Cabane J. Digestive tract involvement in systemic sclerosis. Autoimmun Rev, 2011. 11(1): p. 68-73.

6. Gyger G, Baron M. Gastrointestinal manifestations of scleroderma: recent progress in evaluation, pathogenesis, and management. Curr Rheumatol Rep, 2012. 14(1): p. 22-29.

7. Bodukam V, Hays RD, Maranian P, et al. Association of gastrointestinal involvement and depressive symptoms in patients with systemic sclerosis. Rheumatology (Oxford), 2011. 50(2): p. 330-334.

8. Omair MA, Lee P. Effect of gastrointestinal manifestations on quality of life in 87 consecutive patients with systemic sclerosis. J Rheumatol, 2012. 39(5): p. 992-996.

9. Nagaraja V, McMahan ZH, Getzug T, et al. Management of gastrointestinal involvement in scleroderma. Curr Treatm Opt Rheumatol, 2015. 1(1): p. 82-105.

10. Valenzuela A, Li S, Becker L, et al. Intestinal pseudo-obstruction in patients with systemic sclerosis: an analysis of the Nationwide Inpatient Sample. Rheumatology (Oxford), 2016. 55(4): p. 654-658.

11. Tian XP, Zhang X. Gastrointestinal complications of systemic sclerosis. World J Gastroenterol, 2013. 19(41): p. 7062-7068.

12. Marie I, Ducrotte P, Denis P, et al. Outcome of small-bowel motor impairment in systemic sclerosis-a prospective manometric 5-yr follow-up. Rheumatology (Oxford), 2007. 46(1): p. 150-153.

13. Marie I, Levesque H, Ducrotte P, et al. Manometry of the upper intestinal tract in patients with systemic sclerosis: a prospective study. Arthritis Rheum, 1998. 41(10): p. 1874-1883.

14. Sjolund K, Bartosik I, Lindberg G, et al. Small intestinal manometry in patients with systemic sclerosis. Eur J Gastroenterol Hepatol, 2005. 17(11): p. 1205-1212.

15. Kumar S, Singh J, Kedika R, et al. Role of muscarinic-3 receptor antibody in systemic sclerosis: correlation with disease duration and effects of IVIG. Am J Physiol Gastrointest Liver Physiol, 2016. 310(11): p. G1052-1060.

16. Jaovisidha K, Csuka ME, Almagro UA, et al. Severe gastrointestinal involvement in systemic sclerosis: report of five cases and review of the literature. Semin Arthritis Rheum, 2005. 34(4): p. 689-702.

17. Sallam H, McNearney TA, Chen JD. Systematic review: pathophysiology and management of gastrointestinal dysmotility in systemic sclerosis (scleroderma). Aliment Pharmacol Ther, 2006. 23(6): p. 691-712. 
18. Sjogren RW. Gastrointestinal motility disorders in scleroderma. Arthritis Rheum, 1994. 37(9): p. 1265-1282.

19. Roberts CG, Hummers LK, Ravich WJ, et al. A case-control study of the pathology of oesophageal disease in systemic sclerosis (scleroderma). Gut, 2006. 55(12): p. 1697-1703.

20. Manetti M, Milia AF, Benelli G, et al. The gastric wall in systemic sclerosis patients: a morphological study. Ital J Anat Embryol, 2010. 115(1-2): p. 115-121.

21. Rohrmann CA, Jr., Ricci MT, Krishnamurthy S, et al. Radiologic and histologic differentiation of neuromuscular disorders of the gastrointestinal tract: visceral myopathies, visceral neuropathies, and progressive systemic sclerosis. AJR Am J Roentgenol, 1984. 143(5): p. 933-941.

22. Knowles CH, De Giorgio R, Kapur RP, et al. Gastrointestinal neuromuscular pathology: guidelines for histological techniques and reporting on behalf of the Gastro 2009 International Working Group. Acta Neuropathol, 2009. 118(2): p. 271-301.

23. Code of Conduct of the Dutch federation of Medical Scientific Societies. [Internet]. Available from: http://www.federa.org. Accessed 8 Dec 2014.

24. den Braber-Ymker M, Lammens M, van Putten MJ, et al. The enteric nervous system and the musculature of the colon are altered in patients with spina bifida and spinal cord injury. Virchows Arch, 2017. 470(2): p. 175-184.

25. den Braber-Ymker M, Heijker S, Lammens M, et al. Practical and reproducible estimation of myenteric interstitial cells of Cajal in the bowel for diagnostic purposes. Neurogastroenterol Motil, 2016. 28(8): p. 1261-1267.

26. Hadi AM, Mouchaers KT, Schalij I, et al. Rapid quantification of myocardial fibrosis: a new macrobased automated analysis. Cell Oncol (Dordr), 2011. 34(4): p. 343-354.

27. Schindelin J, Arganda-Carreras I, Frise E, et al. Fiji: an open-source platform for biological-image analysis. Nat Methods, 2012. 9(7): p. 676-682.

28. Overbeek MJ, Vonk MC, Boonstra A, et al. Pulmonary arterial hypertension in limited cutaneous systemic sclerosis: a distinctive vasculopathy. Eur Respir J, 2009. 34(2): p. 371-379.

29. Bernardini N, Ippolito C, Segnani C, et al. Histopathology in gastrointestinal neuromuscular diseases: methodological and ontological issues. Adv Anat Pathol, 2013. 20(1): p. 17-31.

30. Rolle U, Piaseczna-Piotrowska A, Puri P. Interstitial cells of Cajal in the normal gut and in intestinal motility disorders of childhood. Pediatr Surg Int, 2007. 23(12): p. 1139-1152.

31. Wang H, Zhang Y, Liu W, et al. Interstitial cells of Cajal reduce in number in recto-sigmoid Hirschsprung's disease and total colonic aganglionosis. Neurosci Lett, 2009. 451(3): p. 208-211.

32. Sjogren RW. Gastrointestinal features of scleroderma. Curr Opin Rheumatol, 1996. 8(6): p. 569575.

33. Emmanuel A. Current management of the gastrointestinal complications of systemic sclerosis. Nat Rev Gastroenterol Hepatol, 2016. 13(8): p. 461-472.

34. Greydanus MP, Camilleri M. Abnormal postcibal antral and small bowel motility due to neuropathy or myopathy in systemic sclerosis. Gastroenterology, 1989. 96(1): p. 110-115.

35. Cohen S, Fisher R, Lipshutz W, et al. The pathogenesis of esophageal dysfunction in scleroderma and Raynaud's disease. J Clin Invest, 1972. 51(10): p. 2663-2668. 


\section{Chapter 6}

36. Singh J, Mehendiratta V, Del Galdo F, et al. Immunoglobulins from scleroderma patients inhibit the muscarinic receptor activation in internal anal sphincter smooth muscle cells. Am J Physiol Gastrointest Liver Physiol, 2009. 297(6): p. G1206-1213.

37. Kawaguchi Y, Nakamura Y, Matsumoto I, et al. Muscarinic-3 acetylcholine receptor autoantibody in patients with systemic sclerosis: contribution to severe gastrointestinal tract dysmotility. Ann Rheum Dis, 2009. 68(5): p. 710-714.

38. Thoua NM, Derrett-Smith EC, Khan K, et al. Gut fibrosis with altered colonic contractility in a mouse model of scleroderma. Rheumatology (Oxford), 2012. 51(11): p. 1989-1998. 
Intestinal hypomotility in systemic sclerosis 


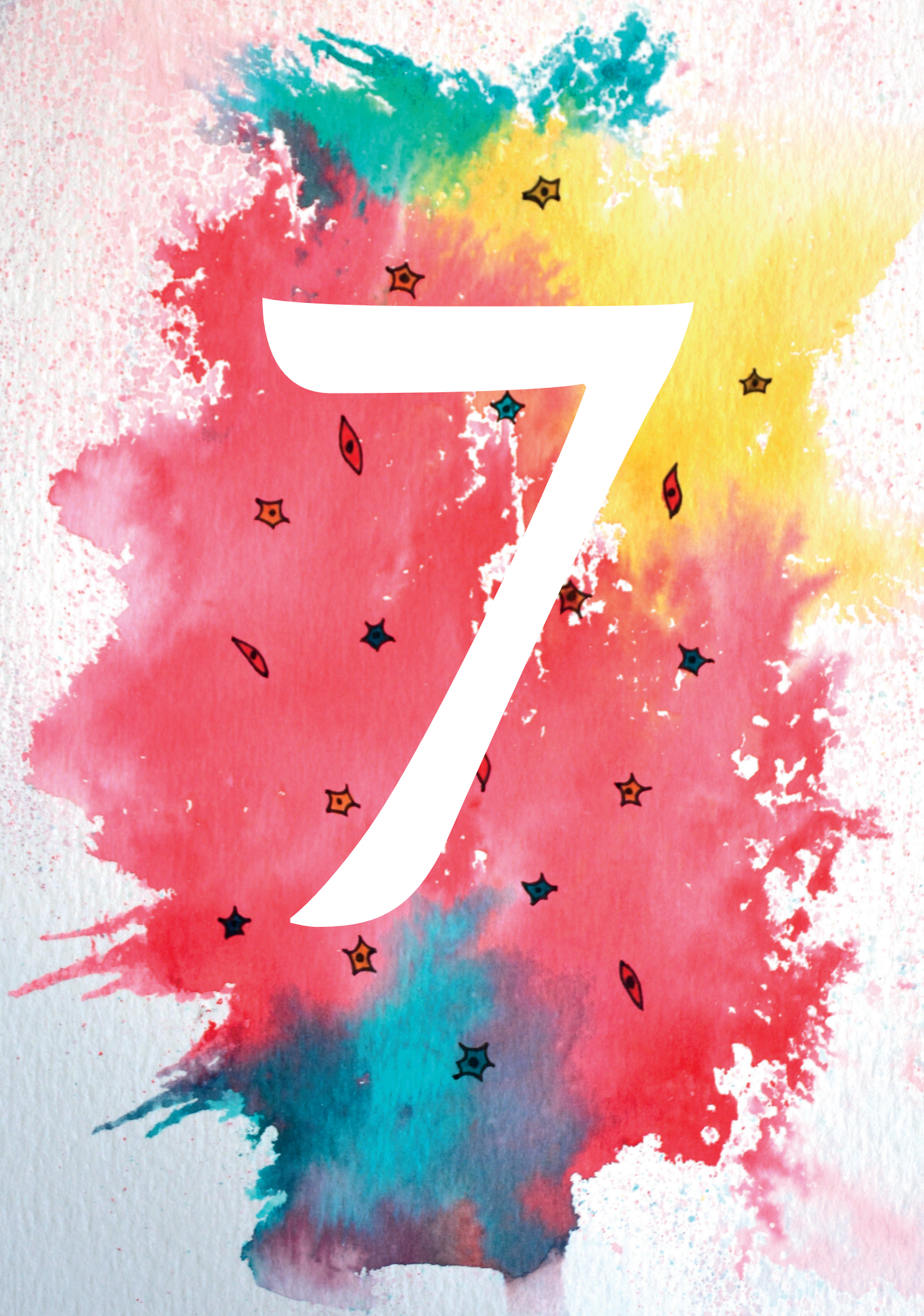




\section{Chapter 7}

\section{General discussion}


Chapter 7 
In this thesis, the histological background of the pathophysiology of secondary motility disorders with a clear aetiology (spinal cord damage, amyloidosis and systemic sclerosis) was investigated to understand possible mechanisms of intestinal motility disorders.

\section{A. Markers for histological evaluation}

A broad panel of histological and immunohistochemical markers was used in this thesis for a systematic analysis of the neuromuscular layers of the bowel wall, based on the markers used in our daily routine diagnostics and the guidelines ${ }^{[1,2]}$. Table 7.1 shows an overview of the markers that I would recommend for use in the clinical workflow of the pathologist for patients with suspected intestinal motility disorders. Based on our results, assessment of the (myenteric) ICC network (CD117) and density of glial cells (S100) seems to be most important in patients with spinal cord damage, amyloidosis and SSc. Other markers can give additional information about abnormalities in the bowel wall, which is essential to provide a complete evaluation of possible causes of dysmotility symptoms in the individual patient.

Calretinin can only be used as marker for a subpopulation of neurons ${ }^{[3]}$ and showed low clinical value in our studies. Calretinin is therefore not recommended for evaluation of enteric neurons. $\mathrm{HuC} / \mathrm{D}$ is a more accurate alternative, because it is a marker for the whole neuron population and moreover suitable for quantification. ${ }^{[2]} \mathrm{I}$ will not advise desmin as marker for smooth muscle tissue, because this marker showed inconsequent staining intensities in our series and provided no clinical differences in our studies. Other smooth muscle markers may be smoothelin, histone deacetylase 8 and smooth muscle myosin heavy chain. ${ }^{[2]}$ 


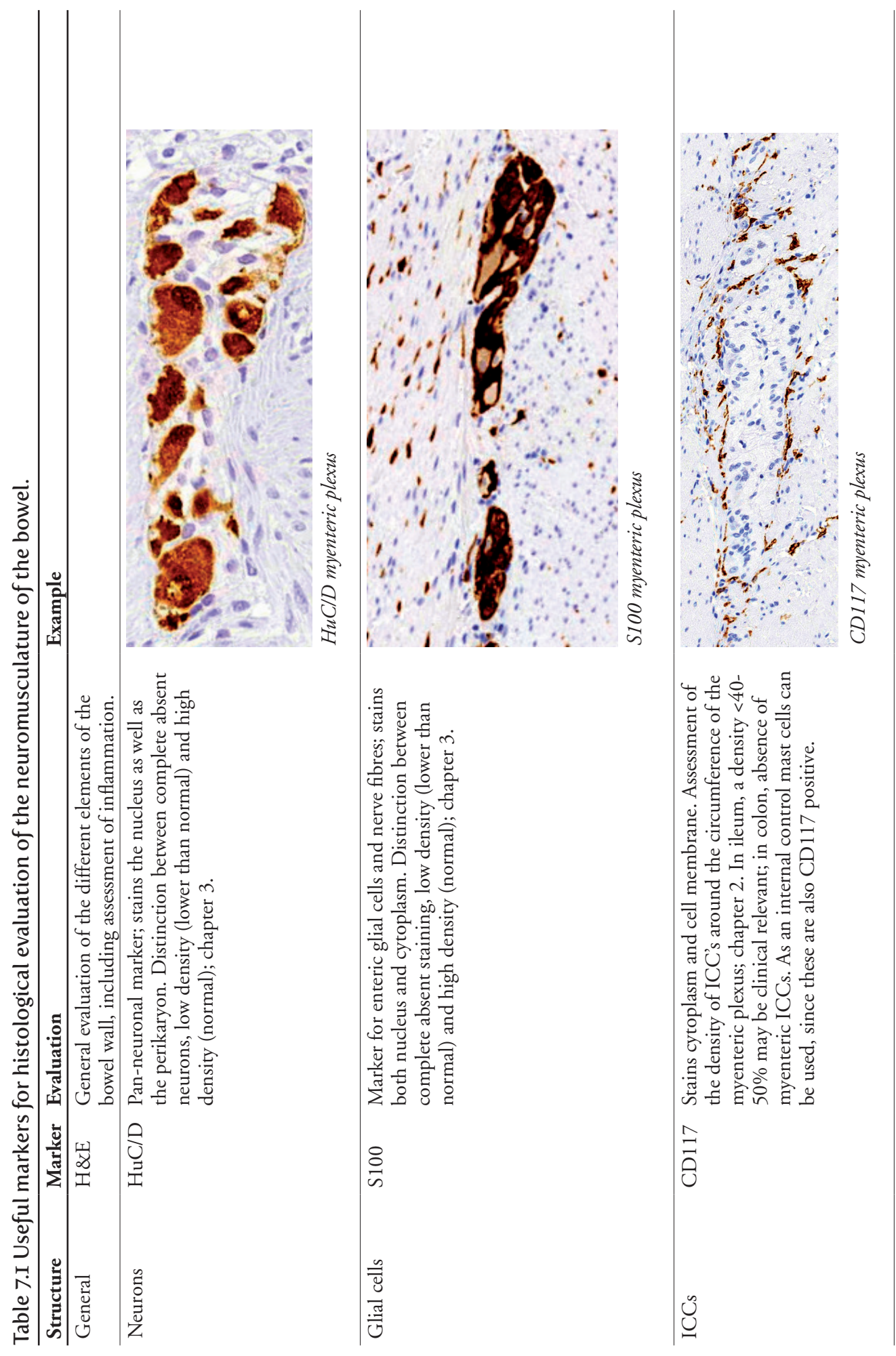



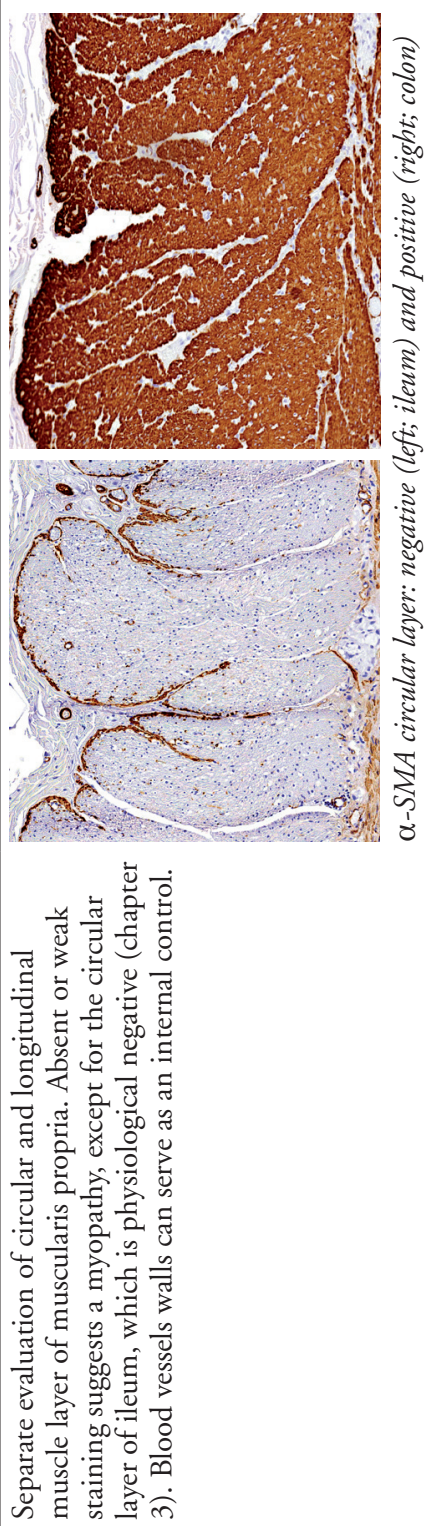

声

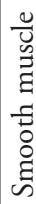

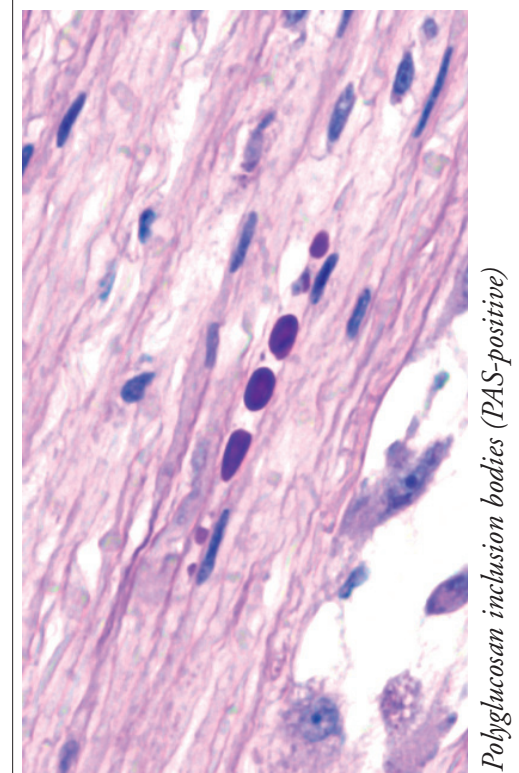

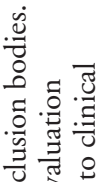

$\exists$ वे

歇

苟. $\Xi$

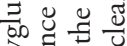

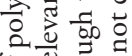

晥总司

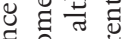

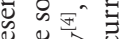

壳害.

吾

壳完号

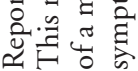

这

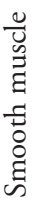




\section{Chapter 7}

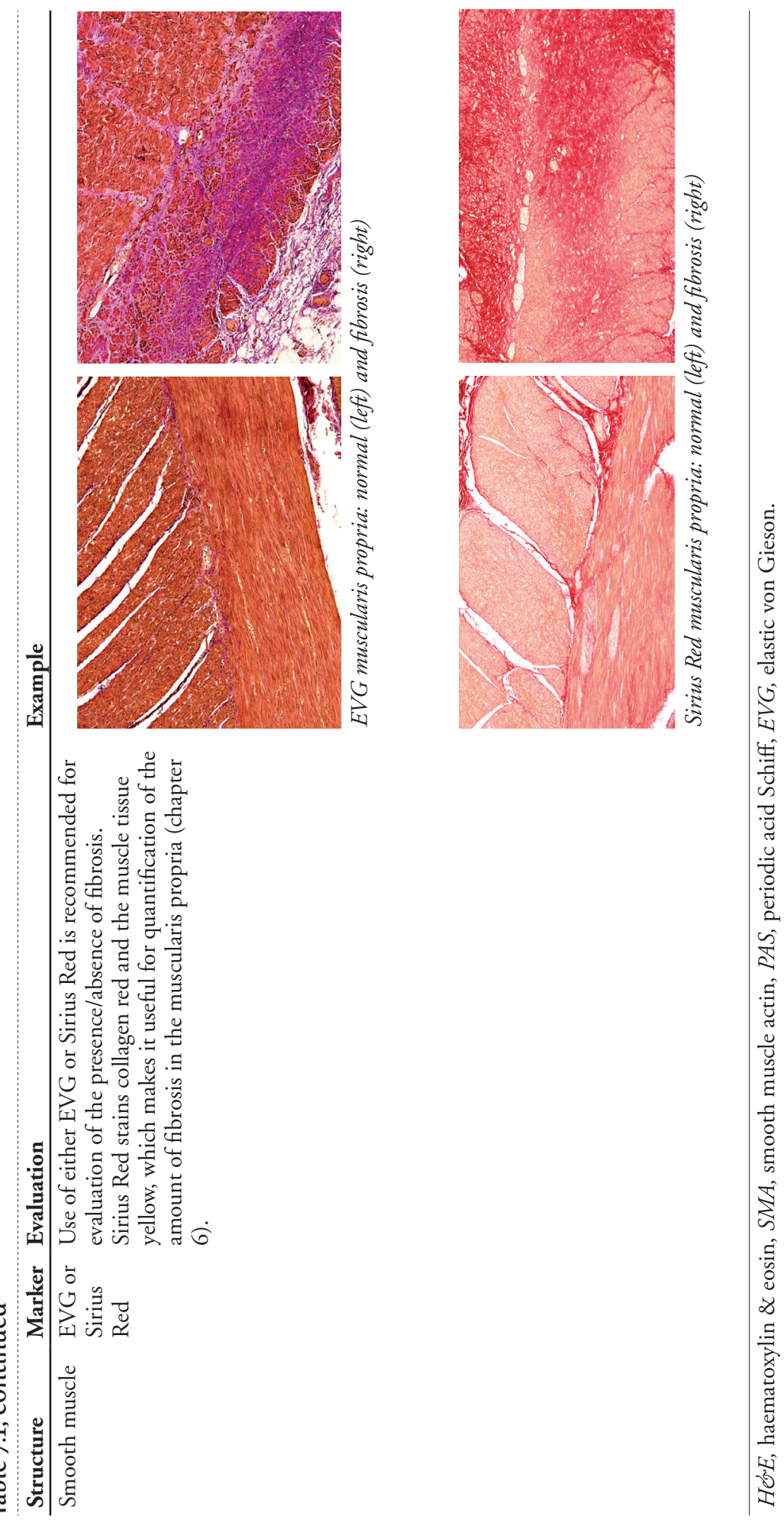




\section{B. Histological comparison of motility disorders}

The London Classification of gastrointestinal neuromuscular pathology (GINMP) provided a classification system based on international consensus. ${ }^{[5]}$ GINMP has been divided into neuropathies, myopathies and ICC abnormalities (enteric mesenchymopathies), each with defined histological phenotypes. Furthermore, this classification describes the relationship between clinical entities (primary and secondary motility disorders) and histological phenotypes. Histological phenotypes were either categorised as aetiological or morphological (associated) changes, in which observed GINMP findings provide strong or weak evidence of a pathogenic mechanism, respectively. ${ }^{[5]}$ We have not distinguished between the aetiological and morphological phenotype. Table 7.2 shows an overview of relevant histological phenotypes of several clinical entities described in literature (Hirschsprung's disease, acquired chronic intestinal pseudo-obstruction (CIPO), slow transit constipation and diabetic gastroenteropathy), supplemented with our results of the presented secondary motility disorders in this thesis. We added vascular involvement to the list of histological findings in Table 7.2 because this was an important finding in amyloidosis and SSc, although the vasculature is strictly speaking not a part of the neuromuscular apparatus. The categories neuropathy, myopathy, ICC abnormalities and vascular involvement (Table 7.2) are discussed below.

Neuropathy. A reduction of neurons (hypoganglionosis) has been shown in several motility disorders: in Hirschsprung's disease (aganglionosis), acquired CIPO, slow transit constipation and in colon samples from patients with diabetic gastroenteropathy. ${ }^{[5-7]}$ We also found hypoganglionosis in the myenteric plexus of patients with spinal cord damage and SSc. In addition, a decrease of glial cells has been reported in slow transit constipation (submucosal and myenteric plexus) ${ }^{[6,8]}$ and was found in our patients with spinal cord damage (myenteric plexus) and SSc (submucosal plexus) as well. In contrast, increased glia were described in patients with acquired CIPO, which seemed to be reactive to neuron loss in the myenteric plexus. ${ }^{[9]}$ We did not find increased glia in our patient groups. Degenerative neuropathy (e.g. shrunken or apoptotic neurons) has been reported in acquired CIPO, slow transit constipation and diabetic gastroenteropathy ${ }^{[5]}$, but degeneration of neurons was not assessed in this thesis. Inflammation of the ganglia (ganglionitis) can be present in Hirschsprung's disease, acquired CIPO and slow transit constipation ${ }^{[5]}$, but was not present in our patient cohorts.

Myopathy. Degenerative leiomyopathy includes myocyte damage and loss, and fibrosis. ${ }^{[5]}$ We found fibrosis in patients with spinal cord damage (longitudinal muscle layer) and SSc (generalised fibrosis in circular layer and focal fibrosis in longitudinal layer). The major cause of muscular failure in our patients with amyloidosis seemed to be deposition of amyloid in the muscularis propria. Degeneration of smooth muscle 
and fibrosis was found in acquired CIPO as well (both muscular layers). ${ }^{[10,11]}$ Significant loss of smooth muscle actin (filament protein abnormality) was only observed in acquired CIPO. ${ }^{[5,12]}$ Some spina bifida and amyloidosis cases in our studies showed lower expression of smooth muscle actin, but no significant differences between patient and control groups could be observed. Polyglucosan inclusion bodies (abnormal content in smooth muscle $e^{[4]}$ ) were found in patients with acquired $\mathrm{CIPO}^{[5]}$, as well as in our patients with amyloidosis (even without association with muscular amyloid deposits), SSc and some control patients, suggesting that this is not a very specific finding.

ICC abnormalities. Decreased ICC network has been described in many clinical entities, including Hirschsprung's disease, acquired CIPO, slow transit constipation and diabetes. ${ }^{[5]}$ Reduction of the myenteric ICC network was seen in all of our patient groups compared to controls, except for colon sections of SSc patients.

Vascular involvement. We investigated the condition of the blood vessels in the intestinal wall in patients with amyloidosis and SSc. In amyloidosis, amyloid deposits were found in the blood vessel walls, while fibrotic changes of small vessels were observed in patients with SSc. Dysfunction of the vasculature may indirectly affect the function of the neuromuscular apparatus, e.g. because of ischaemia.

\section{Pathophysiology of intestinal motility disorders}

The histological characteristics found in our research into secondary motility disorders may provide useful understanding of pathophysiological mechanisms of intestinal motility disorders in general. The two most remarkable histological changes in our studies were reduction of the density of the myenteric ICC network and decrease of glial cell density. Furthermore, a large individual variation with respect to histological features was shown within our patient groups.

\section{Myenteric ICC network}

A decreased ICC network was generally found in our patient cohorts. The myenteric ICC network is important for gut motility, so that loss of ICCs may result in clinical symptoms of intestinal dysmotility. ${ }^{[13,14]}$ By contrast, reduction of ICCs or disruption of the ICC network could be a result instead of the cause of luminal dilatation, as shown in colon samples of mice. ${ }^{[15]}$ Our studies showed heterogeneous reduction of the ICC network in all patient groups, but not in all individual patients. The studies in this thesis may give some valuable insights in this complex research area.

First, we showed that the myenteric ICC network may be the earliest affected component of the enteric neuromusculature after disrupted extrinsic innervation in patients with spinal cord damage (chapter 4). In our patient groups, ICC loss may precede disruption of the distribution of neurons and glial cells in the ENS. However, 
the presence of neurons and glial cells does not imply that the neurotransmission is functional. Impaired neurotransmission from the extrinsic nerves to the ENS may affect the number of ICCs prior to the distribution of neurons and glial cells, probably because of the fine balance of ICC homeostasis. ICC loss may further decrease neurotransmission, which may subsequently lead to reduction of the number of neurons or glial cells. The cooperation between ICCs, neurons and glial cells is extremely complex and have to be further investigated in physiological studies.

Second, we discovered that fibrosis or amyloid was present in (small) blood vessel walls in the intestines of amyloidosis and SSc patients, respectively. We postulate that the resulting decreased oxygenation may lead to ischaemic changes in ICCs, resulting in a decreased ICC network (chapters 5 and 6). In small animals with ischaemia/ reperfusion injury after gut transplantation (short-term ischaemia), apoptosis and recovery of the ICC network have been shown. ${ }^{[16,17]}$ Mei et al. ${ }^{[18]}$ described apoptosis and recovery of ICCs after ischaemia and reperfusion, but in addition demonstrated the presence of apoptosis in enteric neurons and smooth muscle cells, although to a lesser extent. Thacker et al. ${ }^{[19]}$ reported a distortion of glial cells after ischaemia/reperfusion injury, which was associated with damage and loss of enteric neurons. Whether ICCs are more sensitive for ischaemia than for example enteric neurons or smooth muscle cells is currently not clearly described in literature, and needs further investigation. However, ICCs might be able to proliferate after (short-term) ischaemia, while enteric neurons may be permanently lost. Apoptotic ICCs have also been found in normal human colon together with histologically normal ICC networks, which supports the hypothesis that in the normal situation there is a balance between apoptosis and proliferation of ICCs. [20] There may be a continuous replacement of ICCs to maintain normal networks and to provide normal GI motility. ${ }^{[20,21]}$ We propose that long-term ischaemia may occur due to the chronic character of amyloidosis and SSc. This may result in disruption of the apoptosis-proliferation balance of the ICC network.

Third, we frequently found amyloid deposits in the smooth muscle tissue adjacently to the myenteric plexus (chapter 5). This may cause a compression of the ICC network leading to degeneration of ICCs, probably prior to degeneration or loss of neurons or glial cells in the myenteric ganglia. As far as we know, the effect of compression on ICCs is currently not described in literature and is thus subject for further research.

In short, we argue that loss of myenteric ICCs can be reactive to other pathologic events, including disrupted extrinsic innervation, ischaemia or compression of ICCs. ICC loss is commonly shown in GINMDs (Table 7.2) and may thus be a response to other mechanisms instead of the primary event in motility disorders. A wide variety of factors may disturb the balance of ICC homeostasis, including, but not limited to the number of ICCs. 


\section{Enteric glia}

The second common finding in our patient groups was a reduced glial cell density. Glial cells may have an important role in the physiological control of gut functions at multiple levels, including the control of GI motility. ${ }^{[22,23]}$ Bassotti et al. showed loss of glial cells, together with reduction of neurons and ICCs, in colon samples of patients with slow transit constipation. ${ }^{[6]}$ The loss of glial cells, but not of neurons and ICCs, was also described in the terminal ileum of the same patients ${ }^{[8]}$, indicating that glial cells may be involved in (severe) abnormal GI motility. ${ }^{[24]}$ We also observed significant loss of glial cells in the myenteric plexus in patients with spinal cord damage and clinical symptoms of severe dysmotility. The glial density was significantly decreased in the colonic submucosal plexus of our SSc patients as well, which might be related to symptoms of severe dysmotility in some patients.

Constipation is the common finding in human pathological conditions with decreased enteric glial cells. ${ }^{[24]}$ Currently, underlying mechanisms are completely unknown, partly because of limited understanding of the factors that regulate the maintenance of enteric glial cell numbers in the GI tract. ${ }^{[23]}$ Factors resulting in loss of enteric glia are also not well understood. Some animal studies have suggested that age may have an effect on glial cell numbers ${ }^{[25,26]}$, but we did not find this association in our control groups. Loss of glial cells may result in decreased function of the glial network. Impaired function of glial cells may result in neuronal damage, which might be an additional cause of GI dysmotility. ${ }^{[23]}$

Reduction of glial cells together with fewer enteric neurons or ICCs might result in intestinal dysmotility, caused by several mechanisms: impaired support and stabilisation of the ENS, decreased gut neurotransmission, and reduced homeostatic support to the enteric neurons, which may lead to neurodegeneration. ${ }^{[24,27]}$ Impairment of enteric glial cells may thus be an important event in the pathogenesis of intestinal dysmotility.

\section{Individual variation}

Primary GINMP such as CIPO and slow transit constipation show a large individual variation within each clinical entity, resulting in an enormous range of histological characteristics, as shown in Table 7.2. We know that CIPO represents a group of heterogeneous disorders with multiple pathophysiological mechanisms resulting in various morphological phenotypes (e.g. neuropathy, myopathy). ${ }^{[28]}$ This variation makes it impossible to distinguish one general underlying pathophysiological mechanism in CIPO and other primary intestinal motility disorders. We investigated secondary GINMP to create more uniform groups based on known aetiology, thus to reduce the heterogeneity within our study groups. Hence, we expected less individual variation of histological changes within one disease group. Nevertheless, we observed large individual differences within each patient group at histological level. Consequently, we found no 
uniform general pathophysiological patterns that could be applied to primary GINMP. Correlation of histological findings to clinical symptoms is important in clinical practice, but this could partly be done in our studies. GI symptoms were not or incompletely reported in medical files, especially in case histories from more than 15-20 years ago, because less was known about GI symptoms in spinal cord damage, amyloidosis and SSc at that time. In future research, it would be important to prospectively investigate the correlation of clinical symptoms and histological changes in a systematic way. Malagaleda et al. recently showed that small intestinal dysmotility detected by intestinal manometry was often associated with abnormal histological findings, but they could not find a correlation between specific manometric patterns and histopathological findings. ${ }^{[2]]}$ Thus, we believe that evaluation of the neuromuscular structures in the gut wall (at the location of the pseudo-obstruction) remains important for patients with persisting clinical GI symptoms. Assessment of each individual case is necessary because of the large diversity between individuals.

\section{Future perspectives}

Appropriate histopathological diagnosis will contribute to an accurate diagnosis, a better prognosis and more adequate (targeted) therapy. ${ }^{[1]}$ The research described in this thesis provides many future directions to improve the histopathological diagnosis of intestinal motility disorders.

\section{Histological analysis of intestinal motility disorders}

In this thesis, we investigated the myenteric ICC network. The diagnostic value of evaluation of the intramuscular ICC network can be studied in future research, because this network may play a role in the neurotransmission. Furthermore, the diagnostic value of other immunohistochemical smooth muscle markers such as smoothelin, histone deacetylase 8 and smooth muscle myosin heavy chain can be investigated in future research. ${ }^{[2,30]}$

Quantitative evaluation of the ENS can give additional information for diagnosis of GINMP. Knowles et al. ${ }^{[31]}$ reviewed relevant quantification methods and normal values of the human ENS. They could not produce robust normal ranges and described methodological recommendations for quantification of the ENS. Currently, the most suitable quantitative evaluation method of cells in the ENS for translation to routine pathologic diagnostics uses double HuC/D-S100 staining. ${ }^{[2]}$ Future studies have to focus on the usability of quantitative evaluation of cells in the ENS in daily diagnostic practice and should correlate (ab)normal cell numbers to clinical symptoms of the patient. 


\section{Full-thickness tissue material}

Full-thickness excision biopsies of the intestinal wall are needed for a complete assessment of the neuromusculature. One problem in daily diagnostic practice is the lack of intestinal full-thickness tissue material. Current laparoscopic (seromuscular) biopsy methods are relatively invasive, and rarely obtained as a diagnostic tool for patients with severe motility disorders. ${ }^{[32,33]}$ Therefore, alternative diagnostic methods for evaluation of all layers of the gut wall are needed. Alternatives are endoscopic full-thickness biopsies ${ }^{[34-37]}$, optical techniques ${ }^{[38]}$ or use of the appendix ${ }^{[39,40]}$. In my view, endoscopic full-thickness biopsy methods are closest to clinical implementation. The first results have recently been shown from colon, showing that sufficient tissue material for histopathological analysis can be obtained. ${ }^{[37]}$ All layers of the colonic wall could be obtained by creating, clipping and resection of a 'pseudopolyp', without general anaesthesia and complications such as leakage or peritonitis. Endoscopic optical techniques may be promising in the further future, especially full-field optical coherence microscopy, which is the only technique that has been used for imaging of the enteric myenteric plexus in human at this moment. ${ }^{[38]}$ Although this technique has potential of real-time imaging of the intestinal wall, many improvement steps have still to be investigated and realised for use in vivo. Assessment of the appendix as alternative method for enteric motility disorders seems to be restricted to systemic diseases in which neuromuscular structures are generally affected (e.g. type 1 diabetes), both in the entire length of the intestine and in all layers of the gut walls. ${ }^{[39,40]}$ However, the effect of mechanical stress or constipation may be absent in the appendix, which may cause (histological) differences between the appendix and the rest of the intestinal wall. Future studies into the diagnostic value of the appendix in systemic intestinal motility disorders are recommended.

\section{Association between histology, clinical symptoms and physiology}

A histopathological classification has been proposed for diagnosis of GINMP, including three categories: neuropathies, myopathies and mesenchymopathies (ICC abnormalities). ${ }^{[5]}$ I agree with Bassotti and Villanacci ${ }^{47}$ that abnormalities in motility disorders are rarely restricted to one part of the neuromusculature, and that more specific categories like neurogliopathies or neuromyopathies could be included in the classification. ${ }^{[2,24,41]}$ This also means that abnormalities of the enteric neuromusculature must systematically be documented in the pathology reports (neuronal and glial density, myenteric ICC network, presence of fibrosis, condition of muscularis propria). In the future, a more detailed histopathological classification will give the opportunity for more targeted (individual) treatment. However, not all patients with histological abnormalities have clinical symptoms of dysmotility. Asymptomatic patients may have no benefit from therapeutic intervention. Therefore, the relation between histopathology and clinical symptoms is important for prognosis and management of the disease and also for research into disease mechanisms. Consequently, clinical symptoms of dysmotility 
should be systematically registered in medical files, in particular for secondary GINMDs. Abdominal pain, vomiting, constipation, diarrhoea, and weight loss should minimally be reported for the intestines. Information about parenteral nutrition and medication influencing bowel motility function should be registered as well. In the future, better documentation of clinical symptoms in routine practice will allow for a better decision whether therapy is advised, based on the correlation between clinical symptoms and histological changes. In addition, histological findings should be related to physiological measurements of gastrointestinal motility such as manometry (local motility) or transit studies (whole-organ) ${ }^{[42,43]}$, which would lead to better understanding of the pathophysiology of (severe) gastrointestinal dysfunction. Keller et al. ${ }^{[43]}$ provides recommendations about clinical motility testing.

\section{Secondary motility disorders}

Future prospective studies into the histological background of pathophysiological mechanisms in spinal cord damage, amyloidosis and systemic sclerosis should combine a systematic histological evaluation of the neuromuscular structures of the intestinal wall with physiological measurements (colonic manometry or transit studies). Clinical symptoms should be accurately documented, as described above. In this way, clinical symptoms can be supported by physiological abnormalities and morphological changes. Use of surgical resection material or full-thickness biopsies is recommended above autopsy material, because the quality of tissue material is usually better. Moreover, reporting of the exact location of the tissue sample (obtained from the dysmotility area or not) will give valuable input for correlation of the histology and clinical abnormalities.

Quantification of the amount of fibrosis in the muscularis propria in SSc was useful in research setting (chapter 6). The clinical value of quantification of fibrosis have to be further investigated, for instance correlation with clinical symptoms and prognosis. For implementation in daily routine diagnostics, this method should be optimised into an easy-to-use tool. 


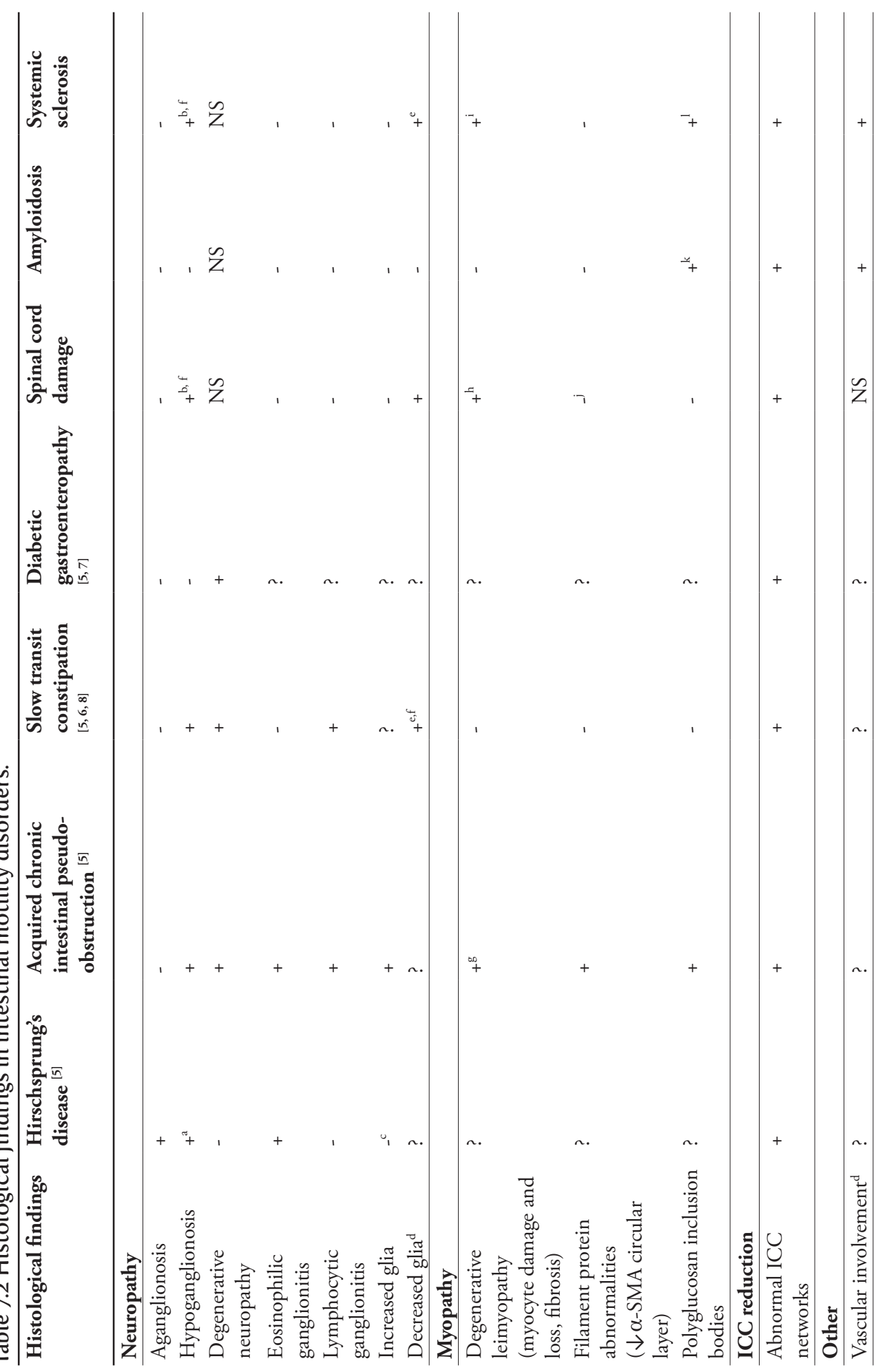




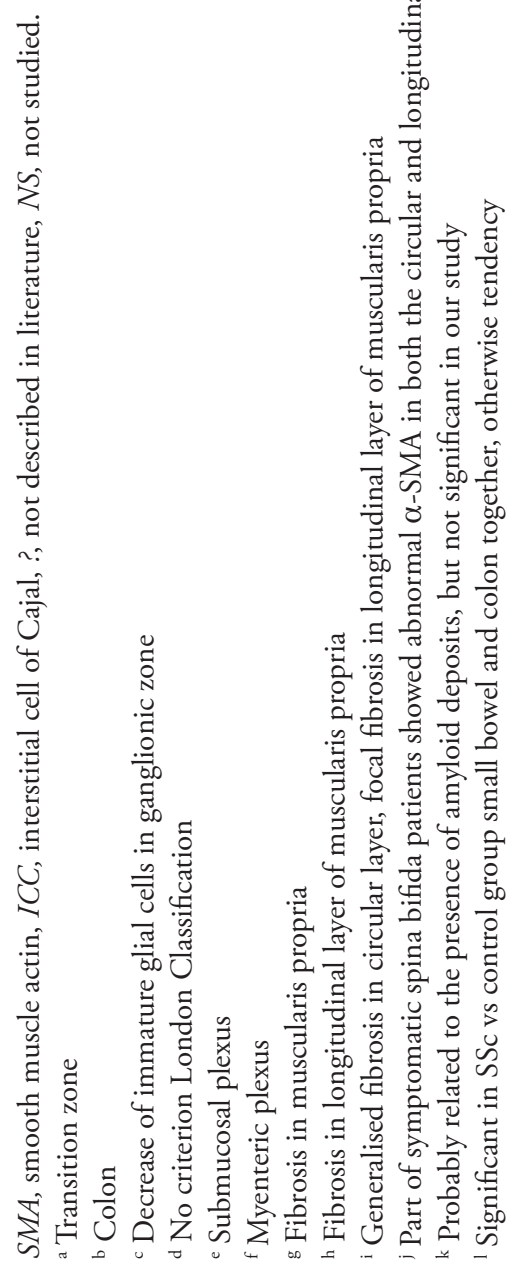




\section{References}

1. Knowles CH, De Giorgio R, Kapur RP, et al. Gastrointestinal neuromuscular pathology: guidelines for histological techniques and reporting on behalf of the Gastro 2009 International Working Group. Acta Neuropathol, 2009. 118(2): p. 271-301.

2. Bernardini N, Ippolito C, Segnani C, et al. Histopathology in gastrointestinal neuromuscular diseases: methodological and ontological issues. Adv Anat Pathol, 2013. 20(1): p. 17-31.

3. Kustermann A, Neuhuber W, Brehmer A. Calretinin and somatostatin immunoreactivities label different human submucosal neuron populations. Anat Rec (Hoboken), 2011. 294(5): p. 858-869.

4. Knowles $\mathrm{CH}$, Nickols CD, Feakins R, et al. A systematic analysis of polyglucosan bodies in the human gastrointestinal tract in health and disease. Acta Neuropathol, 2003. 105(4): p. 410-413.

5. Knowles $\mathrm{CH}$, De Giorgio R, Kapur RP, et al. The London Classification of gastrointestinal neuromuscular pathology: report on behalf of the Gastro 2009 International Working Group. Gut, 2010. 59(7): p. 882-887.

6. Bassotti G, Villanacci V, Maurer CA, et al. The role of glial cells and apoptosis of enteric neurones in the neuropathology of intractable slow transit constipation. Gut, 2006. 55(1): p. 41-46.

7. Chandrasekharan B, Anitha M, Blatt R, et al. Colonic motor dysfunction in human diabetes is associated with enteric neuronal loss and increased oxidative stress. Neurogastroenterol Motil, 2011. 23(2): p. 131-138, e126.

8. Bassotti G, Villanacci V, Cathomas G, et al. Enteric neuropathology of the terminal ileum in patients with intractable slow-transit constipation. Human Pathology, 2006. 37(10): p. 1252-1258.

9. Schuffler MD, Jonak Z. Chronic Idiopathic Intestinal Pseudo-Obstruction Caused by a Degenerative Disorder of the Myenteric Plexus: The Use of Smith's Method to Define the Neuropathology. Gastroenterology, 1982. 82(3): p. 476-486.

10. Amiot A, Cazals-Hatem D, Joly F, et al. The role of immunohistochemistry in idiopathic chronic intestinal pseudoobstruction (CIPO): a case-control study. Am J Surg Pathol, 2009. 33(5): p. 749-758.

11. Moore SW, Schneider JW, Kaschula RDC. Non-familial visceral myopathy: clinical and pathologic features of degenerative leiomyopathy. Pediatric Surgery International, 2002. 18(1): p. 6-12.

12. Knowles CH, Silk DB, Darzi A, et al. Deranged smooth muscle alpha-actin as a biomarker of intestinal pseudo-obstruction: a controlled multinational case series. Gut, 2004. 53(11): p. 15831589.

13. Huizinga JD, Zarate N, Farrugia G. Physiology, Injury, and Recovery of Interstitial Cells of Cajal: Basic and Clinical Science. Gastroenterology, 2009. 137(5): p. 1548-1556.

14. Streutker CJ, Huizinga JD, Driman DK, et al. Interstitial cells of Cajal in health and disease. Part I: normal ICC structure and function with associated motility disorders. Histopathology, 2007. 50(2): p. 176-189.

15. Wu CC, Lin YM, Gao J, et al. Are Interstitial Cells of Cajal Involved in Mechanical Stress-Induced Gene Expression and Impairment of Smooth Muscle Contractility in Bowel Obstruction? PLoS ONE, 2013. 8(9): e76222.

16. von Websky MW, Kalff JC, Schafer N. Current knowledge on regulation and impairment of 
motility after intestinal transplantation. Curr Opin Organ Transplant, 2015. 20(3): p. 303-307.

17. Suzuki M, Takahashi A, Toki F, et al. The effects of intestinal ischemia on colonic motility in conscious rats. Journal of Gastroenterology, 2008. 43(10): p. 767-773.

18. Mei F, Guo S, He YT, et al. Apoptosis of interstitial cells of Cajal, smooth muscle cells, and enteric neurons induced by intestinal ischemia and reperfusion injury in adult guinea pigs. Virchows Archiv, 2009. 454(4): p. 401-409.

19. Thacker M, Rivera LR, Cho H-J, et al. The relationship between glial distortion and neuronal changes following intestinal ischemia and reperfusion. Neurogastroenterology \& Motility, 2011. 23(11): p. e500-e509.

20. Gibbons SJ, De Giorgio R, Faussone Pellegrini MS, et al. Apoptotic cell death of human interstitial cells of Cajal. Neurogastroenterology and Motility, 2009. 21(1): p. 85-93.

21. Gibbons SJ, Gao J, Farrugia G. ICC network density: Regulation and consequences, in Lecture Notes in Computational Vision and Biomechanics. 2013. p. 29-49.

22. Neunlist M, Rolli-Derkinderen M, Latorre R, et al. Enteric glial cells: recent developments and future directions. Gastroenterology, 2014. 147(6): p. 1230-1237.

23. Sharkey KA. Emerging roles for enteric glia in gastrointestinal disorders. J Clin Invest, 2015. 125(3): p. 918-925.

24. Bassotti G, Villanacci V, Fisogni S, et al. Enteric glial cells and their role in gastrointestinal motor abnormalities: Introducing the neuro-gliopathies. World Journal of Gastroenterology, 2007. 13(30): p. 4035-4041.

25. Phillips RJ, Kieffer EJ, Powley TL. Loss of glia and neurons in the myenteric plexus of the aged Fischer 344 rat. Anatomy and Embryology, 2004. 209(1): p. 19-30.

26. McClain JL, Grubišić V, Fried D, et al. Ca2+ responses in enteric glia are mediated by connexin-43 hemichannels and modulate colonic transit in mice. Gastroenterology, 2014. 146(2): p. 497-507.

27. Ruhl A. Glial cells in the gut. Neurogastroenterol Motil, 2005. 17(6): p. 777-790.

28. Downes TJ, Cheruvu MS, Karunaratne TB, et al. Pathophysiology, Diagnosis, and Management of Chronic Intestinal Pseudo-Obstruction. J Clin Gastroenterol, 2018. 52(6): p. 477-489.

29. Malagelada C, Karunaratne TB, Accarino A, et al. Comparison between small bowel manometric patterns and full-thickness biopsy histopathology in severe intestinal dysmotility. Neurogastroenterology and Motility, 2018. 30(3): e13219.

30. Wedel T, Van Eys GJ, Waltregny D, et al. Novel smooth muscle markers reveal abnormalities of the intestinal musculature in severe colorectal motility disorders. Neurogastroenterol Motil, 2006. 18(7): p. 526-538.

31. Knowles $\mathrm{CH}$, Veress B, Kapur RP, et al. Quantitation of cellular components of the enteric nervous system in the normal human gastrointestinal tract--report on behalf of the Gastro 2009 International Working Group. Neurogastroenterol Motil, 2011. 23(2): p. 115-124.

32. Knowles $\mathrm{CH}$, Martin JE. New techniques in the tissue diagnosis of gastrointestinal neuromuscular diseases. World J Gastroenterol, 2009. 15(2): p. 192-197.

33. King SK, Sutcliffe JR, Hutson JM. Laparoscopic seromuscular colonic biopsies: a surgeon's experience. J Pediatr Surg, 2005. 40(2): p. 381-384. 


\section{Chapter 7}

34. Ohlsson B, Gustafsson RJ, Toth E, et al. Endoscopic versus Laparoscopic Full-Thickness Biopsy in the Pathological Evaluation of the Enteric Nervous System. Case Rep Gastroenterol, 2018. 12(1): p. 32-40.

35. Rajan E, Al-Bawardy B, Gostout CJ, et al. Endoscopic muscle biopsy sampling of the duodenum and rectum: a pilot survival study in a porcine model to detect myenteric neurons. Gastrointestinal endoscopy, 2018. 87(2): p. 600-606.

36. Rajan E, Gostout CJ, Wong Kee Song LM, et al. Innovative gastric endoscopic muscle biopsy to identify all cell types, including myenteric neurons and interstitial cells of Cajal in patients with idiopathic gastroparesis: a feasibility study (with video). Gastrointestinal endoscopy, 2016. 84(3): p. 512-517.

37. Valli PV, Pohl D, Fried M, et al. Diagnostic use of endoscopic full-thickness wall resection (eFTR)-a novel minimally invasive technique for colonic tissue sampling in patients with severe gastrointestinal motility disorders. Neurogastroenterol Motil, 2018. 30(1): e13153.

38. Coron E, Auksorius E, Pieretti A, et al. Full-field optical coherence microscopy is a novel technique for imaging enteric ganglia in the gastrointestinal tract. Neurogastroenterology \& Motility, 2012. 24(12): p. e611-e621.

39. Knowles $\mathrm{CH}$, De Giorgio R. Observations on a vestigial organ: a potential surrogate for enteric neuromesenchymal disease. Neurogastroenterol Motil, 2008. 20(4): p. 263-268.

40. Abell TL, Camilleri M, Donohoe K, et al. Consensus Recommendations for Gastric Emptying Scintigraphy: A Joint Report of the American Neurogastroenterology and Motility Society and the Society of Nuclear Medicine. Am J Gastroenterol, 2008. 103(3): p. 753-763.

41. Bassotti G, Villanacci V. The London Classification of gastrointestinal neuromuscular pathology: a little more flexibility would be wise. Gut, 2011. 60(10): p. 1437.

42. Emmanuel A, Butt S. Small intestine and colon motility. Medicine, 2015. 43(5): p. 271-275.

43. Keller J, Bassotti G, Clarke J, et al. Advances in the diagnosis and classification of gastric and intestinal motility disorders. Nature Reviews Gastroenterology \&Amp; Hepatology, 2018. 15: p. 291-308. 
General discussion 


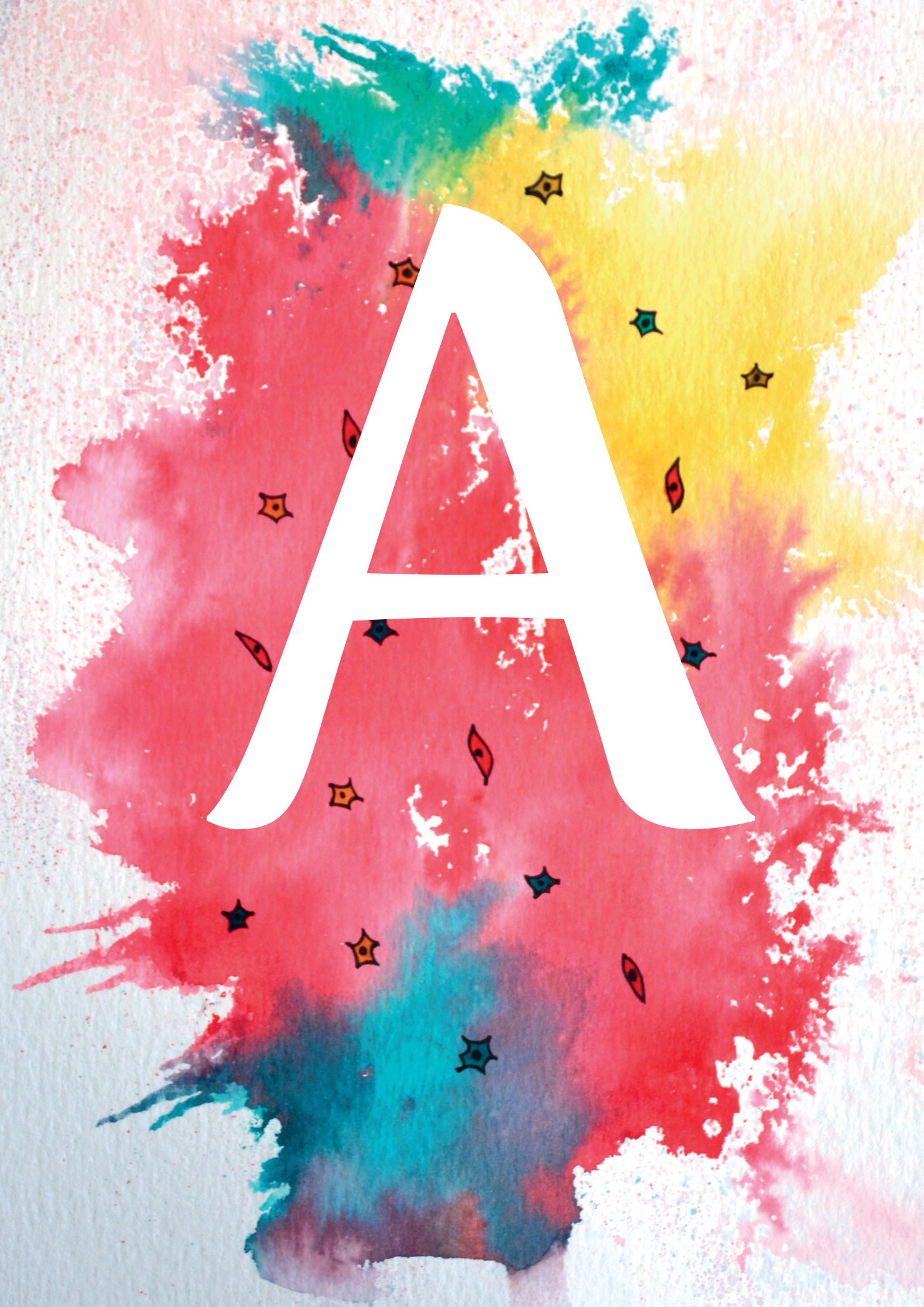




\section{Appendices}

\section{Summary}

Nederlandse samenvatting

List of abbreviations

List of publications

List of abstracts and presentations

Curriculum Vitae

Dankwoord 
Appendices 


\section{Summary}

Intestinal motility is regulated by the enteric nervous system (ENS), the interstitial cells of Cajal (ICCs) and the smooth muscle cells of the muscularis propria. Changes in one or more of these structures can lead to disruption of intestinal motility, summarised in the term gastrointestinal neuromuscular disease (GINMD). The term GINMD includes a clinical heterogeneous group of both primary motility disorders, in which the intestinal neuromuscular system is primarily affected (e.g. Hirschsprung's disease or chronic intestinal pseudo-obstruction), and secondary motility disorders, which are the result of a systemic disease (e.g. systemic sclerosis or amyloidosis). Primary GINMDs such as chronic intestinal pseudo-obstruction or slow transit constipation show a large individual variation, resulting in a broad spectrum of histological characteristics in the bowel. This makes it impossible to distinguish one general underlying pathophysiologic mechanism. The aetiology of secondary GINMDs is generally better understood, but the histopathological features are often not systematically studied.

In this thesis, the histological background of the pathophysiology of secondary motility disorders with a clear aetiology (spinal cord damage, amyloidosis and systemic sclerosis) is investigated to understand possible mechanisms of intestinal motility disorders.

\section{Part I: Methodological issues}

The myenteric network of ICCs may have an important role in peristalsis and propagates slow waves to smooth muscle cells. Loss of ICCs may therefore result in intestinal dysmotility. The International Working Group on GINMD suggested in their guidelines the importance of histological assessment of ICCs in the bowel for diagnosing patients with GINMD. Reporting a decrease in ICC number of more than $50 \%$ might be useful in diagnostic workup, according to quantitative methods. However, the quantitative methods described in literature are not practical for the daily routine of the pathologist. Therefore, we presented a straightforward semiquantitative estimation method for myenteric ICCs of the bowel (chapter 2). In this study, two control groups and four groups composed of patients with gastrointestinal motility disorders were created. The proposed estimation method for the myenteric ICC network generally showed good interobserver and intra-observer agreement and reliability. More myenteric ICCs were found in the small bowel than in the colon. No significant differences between colonic regions were shown, nor were there any differences in the orientation of the sections. Since this method is semiquantitative, simple and capable to differentiate between normal and diseased tissue, it can be used in routine diagnostics of gastrointestinal neuromuscular disorders. 
Besides the ICC network, other neuromuscular structures were semiquantitatively evaluated in this thesis using different immunohistochemical stains, including the number of neurons, the density of the nerve fibre network (glial cells), and smooth muscle filament proteins (chapter 3). These semiquantitative methods were used in the studies presented in chapters 4 to 6.

\section{Part II: Secondary motility disorders}

A graphic summary of the most important histological findings of spinal cord damage, amyloidosis and systemic sclerosis is given in Figure A.1.

\section{Spinal cord damage}

Spina bifida and spinal cord injury are both diseases with a dysfunctional spinal cord, resulting in interruption of the extrinsic nerve fibres of bowel innervation. Extrinsic modulation of the regulation of enteric motility by the ENS is needed for adequate bowel activity. Therefore, damaged extrinsic input results in enteric dysmotility. In the colon, two main types of dysfunction can be distinguished, dependent on the level of the lesion: an upper motor neuron syndrome and a lower motor neuron syndrome. Both syndromes result in constipation. In chapter 4, we studied colon samples from gut resections and could distinguish between a group with severe motility disorders (as indication for surgery), and a group with mild or without motility disorders (other indications for surgery, unavailable information about dysmotility related symptoms).

The disruption of extrinsic innervation of the intestines, due to spinal cord damage, results in disturbance of modulation of the intrinsic reflexes of the ENS. Therefore, we expected changes in the ENS, specifically alterations in the distribution of neurons and/or glial cells. Our study showed a reduction of the myenteric ICC network in almost all spina bifida and spinal cord injury patients, but a reduction of neuronal density in approximately two thirds of the patients with ICC loss. Therefore, the ICC network may be the first structure of the neuromuscular apparatus that becomes affected by damaged extrinsic innervation. Reduction of ICCs may precede loss of neurons and glial cells in the myenteric plexus. Interestingly, enteric glia were significantly decreased in patients with severe motility problems, indicating that glial cells play a significant role in the regulation of motility, as described in literature. No differences could be found between spina bifida (congenital disease) and spinal cord injury (acquired disease) patients, suggesting that changes in the neuromuscular apparatus are reactive to disrupted extrinsic innervation regardless of the aetiology of the lesions. 


\section{Amyloidosis}

Amyloidosis includes a diverse group of disorders characterised by extracellular abnormal fibrillar deposits in various tissues. In chapter 5 , we studied gut samples from patients with AL and AA amyloidosis, the two most common types of amyloidosis. AL amyloidosis is associated with plasma cell dyscrasias, producing increased immunoglobulin light chain amyloid fibrils. The underlying diseases of AA amyloidosis are chronic inflammatory or infectious disorders (i.e. rheumatoid arthritis and Crohn's disease), resulting in abnormal levels of serum amyloid $\mathrm{A}$, which is an acute-phase reactant protein produced by the liver. In both AL and AA amyloidosis, insoluble amyloid proteins can be deposited in several internal organs including the GI tract.

It has been suggested that different mechanisms are responsible for intestinal pseudo-obstruction in those patients: an enteric myopathy in AL and a neuropathy in AA amyloidosis. Furthermore, amyloid deposits are frequently found in the vascular walls, which may lead to ischaemia and further damage of neuromuscular structures in the gut wall. We therefore expected muscular changes in AL amyloidosis, neuronal changes in AA amyloidosis, and vascular involvement in both types of amyloidosis. In the mucosa, amyloid deposits were present in AA but absent in AL patients. Deposition of amyloid was present in the vascular walls of all patients as was expected. We found no differences between the AL and AA amyloidosis groups in the muscle layers and nerve plexuses of the bowel wall. Based on our histological findings, we proposed an alternative hypothesis about intestinal dysmotility in amyloidosis. First, amyloid is deposited in blood vessel walls. Second, amyloid deposition extends into the muscularis propria. Third, this may affect the myenteric ICC network leading to ICC loss. Finally, the myenteric plexus may become involved, possibly resulting in clinical symptoms of severe dysmotility (e.g. pseudo-obstruction).

\section{Systemic sclerosis}

In chapter 6, we investigated histological features of hypomotility in systemic sclerosis (SSc). SSc is a complex connective tissue disease caused by unknown environmental factors resulting in accumulation of collagen in the skin and visceral organs, including the GI tract. Although the exact mechanisms are still unclear, several processes play a role in the pathogenesis of SSc: an abnormal immune system leads to production of autoantibodies and autoimmunity, microvasculopathy, and fibroblast dysfunction resulting in excessive fibrosis. Involvement of the intestines can lead to hypomotility. Processes involved in the intestinal pathogenesis are microvascular damage, dysfunction of autonomic nerves, muscular atrophy and fibrosis, and autoantibodies, although it is currently not clear how these mechanisms interact. Microvascular damage and ischaemia have been suggested as the primary event in intestinal involvement.

It has been postulated that development of intestinal dysmotility may be a sequential process, starting with vascular changes, leading to neuronal damage, atrophy 
of the muscularis propria, followed by fibrosis. Hence, we expected in tissue material from SSc patients with fibrosis in the muscularis propria (the end-stage of the process of intestinal dysmotility) that vascular changes and neuronal damage could be observed as well. Our results could only partly confirm this, indicating that several parallel instead of sequential mechanisms may occur, resulting in large variation of morphological characteristics between individual patients. The diverse histological characteristics between patients may explain the variety of clinical intestinal symptoms in patients with SSc. The key finding of our histological study into SSc was the presence of fibrosis in blood vessel walls and in the muscularis propria, especially in the circular layer of the muscularis propria. In small bowel sections of SSc patients with severe intestinal dysmotility complaints, we did not find an association with histological changes of the neuromusculature. In the colon, reduction of the ENS may explain the symptoms of severe colonic dysmotility in some patients.

\section{General conclusion}

Finally, the results and conclusions of this thesis are discussed in a broader perspective. The two most striking findings in our studies on secondary motility disorders are a decreased density of the myenteric ICC network and a reduced density of glial cells in a large part of our patient cohorts. By limiting ourselves to well-defined secondary motility disorders, we have aimed for groups that are as uniform as possible, in contrast to the heterogeneous group of primary GINMDs. The study into specific diseases has provided new insights and hypotheses within the field of GINMDs. The surprising heterogeneity within our patient cohorts makes it difficult to derive general pathophysiological mechanisms for (primary) intestinal motility disorders. Therefore, it remains important to assess the neuromuscular structures in the gut of each individual patient with severe clinical motility complaints. A careful histopathological diagnosis will ultimately contribute to an accurate diagnosis, a better prognosis and appropriate (targeted) therapy for the patient. 

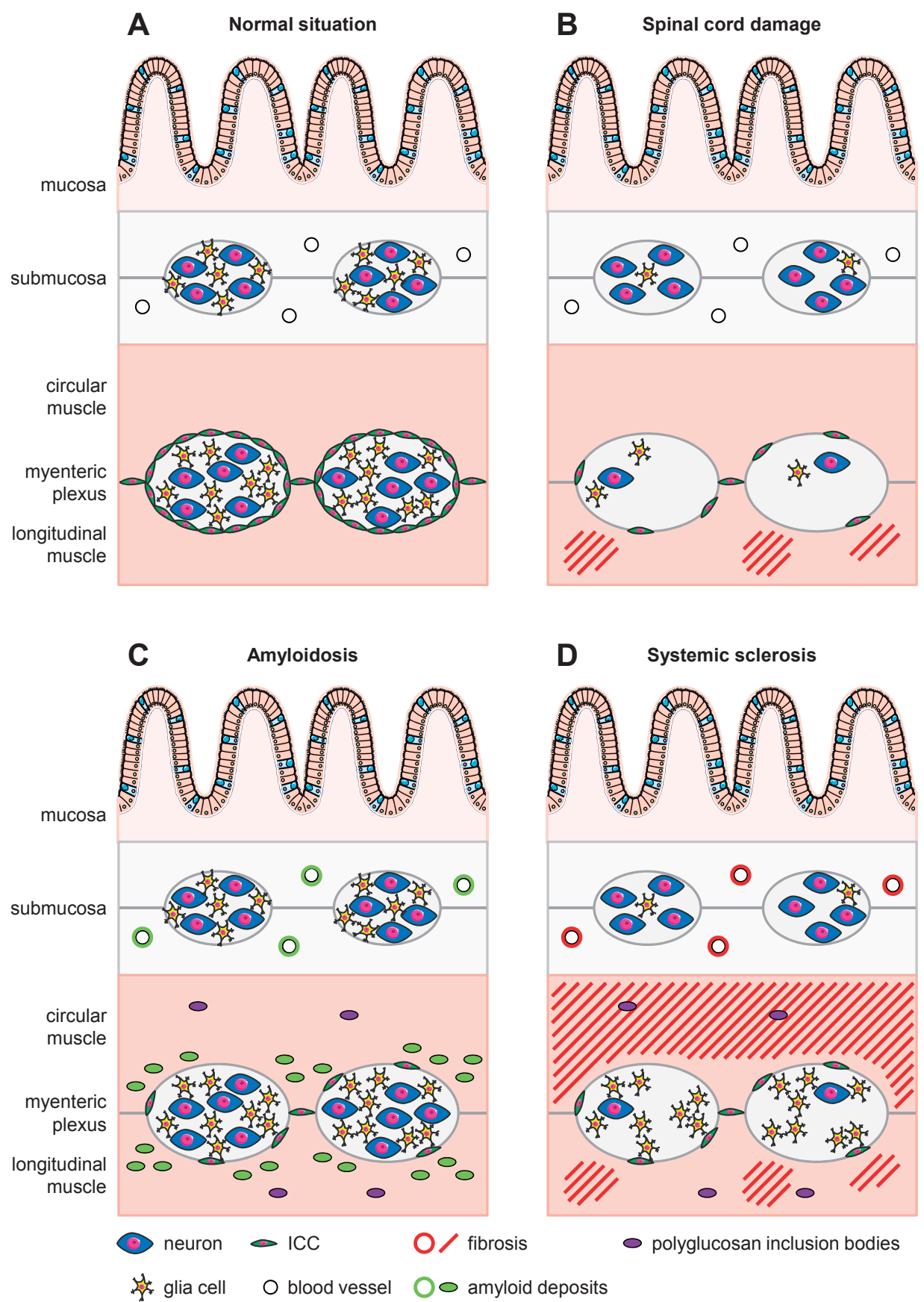

Figure A.I Graphic summary of the main histological findings in secondary motility disorders. A) Schematic representation of the gut wall in normal condition. Only structures studied in this thesis are shown: submucosal and myenteric plexuses, myenteric ICC network, muscularis propria and blood vessels. B) Major histological changes in spinal cord damage were decreased neurons in the myenteric plexus 


\section{Appendices}

(colon), loss of glial cells, fibrosis in the longitudinal muscle layer and reduction of the myenteric ICC network. C) In amyloidosis, amyloid deposits were found in the vascular walls (AL and AA patients) and in the muscularis propria (AL patients). Polyglucosan inclusion bodies were present in the muscularis propria, and a reduction of ICCs was shown. D) Findings in systemic sclerosis were fibrosis in the blood vessel walls, and in the circular and longitudinal muscle layers. Neurons were decreased in the myenteric plexus (colon), loss of glial cells were shown in the submucosal plexus, polyglucosan inclusion bodies were present in the muscularis propria, and the ICC network was reduced. ICC, interstitial cell of Cajal. This figure is created with images adapted from Servier Medical Art (Intestinal villi; Neuron). Original images are licensed under a Creative Commons Attribution 3.0 Unreported License. Simplification and colour changes were made to the original neuron and mucosa cartoons. 


\section{Nederlandse samenvatting}

Darmmotiliteit wordt gereguleerd door het enterische zenuwstelsel (Engels: enteric nervous system, ENS), de interstitiële cellen van Cajal (ICC's) en de gladde spiercellen van de muscularis propria. Afwijkingen in een of meer van deze structuren kunnen leiden tot een verstoring van de darmmotiliteit, samengevat onder de noemer gastro-intestinale neuromusculaire ziekte (GINMD). De term GINMD omvat een klinische heterogene groep van zowel primaire motiliteitsstoornissen, waarbij het neuromusculaire systeem van de darm primair is aangedaan (bijvoorbeeld ziekte van Hirschsprung of chronische intestinale pseudo-obstructie), als secundaire motiliteitsstoornissen, die het gevolg zijn van een systemische ziekte (bijvoorbeeld systemische sclerose of amyloïdose). Primaire GINMD's zoals chronische intestinale pseudo-obstructie of slow-transit obstipatie tonen een grote individuele variatie, waarbij een breed spectrum aan histologische kenmerken in de darm wordt gezien. Hierdoor is het onmogelijk om één algemeen onderliggend pathofysiologisch mechanisme te onderscheiden. De etiologie van secundaire GINMD's is over het algemeen duidelijker, maar de histopathologische kenmerken zijn meestal niet systematisch onderzocht.

In dit proefschrift wordt de histologische achtergrond van de pathofysiologie van secundaire motiliteitsstoornissen met een duidelijke etiologie (beschadiging van het ruggenmerg, amyloïdose en systemische sclerose) onderzocht om mogelijke mechanismen van darmmotiliteitsstoornissen te begrijpen.

\section{Deel I: Methodologische issues}

Het myenterische netwerk van ICC's heeft een belangrijke rol in de peristaltiek en zorgt onder andere voor de voortplanting van 'slow waves' naar de gladde spiercellen. Verlies van ICC's kan daarom leiden tot dismotiliteit van de darm. De Internationale Werkgroep voor GINMD beschrijft in haar richtlijnen het belang van histologische beoordeling van ICC's in de darm voor de diagnose van patiënten met GINMD. Het aantal ICC's kan op een kwantitatieve manier worden bepaald, waarbij het vermelden van een afname met meer dan 50\% nuttig kan zijn in de diagnostiek. De kwantitatieve methoden die in de literatuur worden gebruikt zijn echter momenteel niet praktisch voor de dagelijkse routine van de patholoog. Daarom presenteerden we in hoofdstuk 2 een eenvoudige semikwantitatieve methode om de ICC's in de myenterische plexus van de darm te schatten. In deze studie werden twee controlegroepen en vier groepen bestaande uit patiënten met gastro-intestinale motiliteitsstoornissen gevormd. De voorgestelde schattingsmethode voor het myenterische ICC-netwerk toonde over het algemeen een goede interobserver- en intra-observerovereenkomst en een goede betrouwbaarheid. Er werden meer myenterische ICC's gevonden in de dunne darm 
dan in de dikke darm. Tussen verschillende regio's in het colon werden geen significante verschillen gevonden. Er waren ook geen verschillen tussen de oriëntatie van de coupes (dwars- of lengtedoorsnede van de darm). Aangezien de beschreven methode eenvoudig en semikwantitatief in staat is om onderscheid te maken tussen normaal en ziek weefsel, kan deze worden gebruikt bij routinematige diagnostiek van GINMD.

Hoofdstuk 3 beschrijft op welke semikwantitatieve manier het aantal neuronen, de dichtheid van het zenuwvezelnetwerk (inclusief gliacellen) en het gladde spierweefsel in de muscularis propria zijn geëvalueerd. Deze methoden zijn gebruikt in de studies in de hoofdstukken 4 tot en met 6 .

\section{Deel II: Secundaire motiliteitsstoornissen}

Een schematisch overzicht van de belangrijkste histologische bevindingen bij beschadiging van het ruggenmerg, amyloïdose en systemische sclerose wordt gegeven in Figuur A.1.

\section{Beschadiging van het ruggenmerg}

Spina bifida en dwarslaesie zijn aandoeningen waarbij het ruggenmerg beschadigd is. Dit resulteert in een onderbreking van de extrinsieke zenuwvezels van de darminnervatie. Voor een goede darmmotiliteit is zowel intrinsieke innervatie (ENS) als extrinsieke innervatie nodig. De intrinsieke peristaltische reflex wordt gemoduleerd door extrinsieke zenuwvezels. Beschadiging van deze extrinsieke input kan daarom resulteren in dismotiliteit van de darm. In de dikke darm kan dit leiden tot obstipatie. In hoofdstuk $\mathbf{4}$ hebben we dikke darmweefsel van patiënten met spina bifida en dwarslaesie onderzocht. We hebben onderscheid gemaakt tussen een groep met ernstige motiliteitsstoornissen (als indicatie voor de operatie) en een groep met lichte of zonder motiliteitsstoornissen (andere indicaties voor chirurgie, informatie over andere symptomen gerelateerd aan dismotiliteit was niet beschikbaar).

In deze patiëntengroepen verwachtten we veranderingen in het ENS, voornamelijk veranderingen in de dichtheid van neuronen en/of gliacellen. In onze studie werd vooral afname van het myenterische ICC-netwerk gevonden bij bijna alle spina bifida en dwarslaesie patiënten, maar slechts een vermindering van de dichtheid van neuronen bij ongeveer tweederde van deze patiënten. Het ICC-netwerk zou daarom de eerste structuur van het neuromusculaire apparaat kunnen zijn die wordt aangedaan als gevolg van beschadigde extrinsieke innervatie. Vervolgens zou de afname van ICC's kunnen resulteren in een verlies van neuronen en gliacellen in de myenterische plexus. Daarnaast zagen we bij patiënten met ernstige motiliteitsproblemen een significante afname van enterische gliacellen. Dit kan wijzen op de relevante rol die gliacellen spelen bij de regulatie van motiliteit, zoals beschreven in de literatuur. Er konden geen verschillen 
worden gevonden tussen patiënten met spina bifida (aangeboren ziekte) en dwarslaesie (verworven ziekte), wat suggereert dat veranderingen in het neuromusculaire apparaat een gevolg zijn van verstoorde extrinsieke innervatie onafhankelijk van etiologie.

\section{Amyloïdose}

Amyloïdose omvat een diverse groep stapelingsziekten van verkeerd gevouwen eiwitten (amyloïd) in verschillende weefsels. In hoofdstuk 5 bestudeerden we darmweefsel van patiënten met $\mathrm{AL}$ - en $\mathrm{AA}$-amyloïdose, de twee meest voorkomende soorten amyloïdose. AL-amyloïdose is het gevolg van groei van plasmacellen in het beenmerg dat resulteert in een hoge productie van immunoglobulines. De onderliggende ziekten van AA-amyloïdose zijn chronische ontstekings- of infectieziekten (zoals reumatoïde artritis en de ziekte van Crohn). Hierdoor wordt een verhoogd niveau van het ontstekingseiwit serum amyloïd A geproduceerd door de lever. Zowel bij AL- als bij AA-amyloïdose kunnen onoplosbare eiwitten in allerlei inwendige organen neerslaan als amyloïd, waaronder het maagdarmkanaal.

In de literatuur wordt beschreven dat verschillende mechanismen verantwoordelijk zijn voor intestinale pseudo-obstructie bij amyloïdosepatiënten: een enterische myopathie in AL-amyloïdose en een neuropathie in AA-amylö̈dose. Daarnaast worden amyloïdafzettingen vaak aangetroffen in de vaatwanden van beide typen amyloïdose, wat kan leiden tot ischemie en verdere beschadiging van neuromusculaire structuren in de darmwand. We verwachtten daarom spierveranderingen in AL-amyloïdose, neurale veranderingen in AA-amylö̈dose en amyloïdneerslag in bloedvaten bij beide typen amyloïdose. In het slijmvlies waren amyloïdafzettingen aanwezig in AA-patiënten maar afwezig in AL-patiënten. Depositie van amyloïd was aanwezig in de vaatwanden van alle patiënten zoals we hadden verwacht. Er werden geen verschillen gevonden tussen de AL- en AA-amylö̈dosegroepen in de spierlagen en zenuwplexussen van de darmwand. Op basis van onze histologische bevindingen, hebben we een alternatieve hypothese voorgesteld over het proces van intestinale dismotiliteit bij amylö̈dose: Amylö̈dafzetting vindt als eerste plaats in de bloedvatwanden. Daarna slaat het amyloïd neer in de muscularis propria. Dit kan vervolgens van invloed zijn op het functioneren van het myenterische ICC-netwerk, leidend tot ICC-verlies. Ten slotte kan de myenterische plexus betrokken raken, wat zou kunnen resulteren in klinische symptomen van ernstige dismotiliteit (bijv. pseudo-obstructie).

\section{Systemische sclerose}

In hoofdstuk $\mathbf{6}$ hebben we histologische kenmerken van verminderde darmmotiliteit bij systemische sclerose (SSc) onderzocht. SSc is een complexe bindweefselaandoening waarvan de oorzaak onbekend is. De ziekte resulteert in ophoping van collageen in de huid en viscerale organen, inclusief het maagdarmkanaal. Verschillende processen spelen een rol bij de pathogenese van SSc: een afwijkend immuunsysteem leidt tot de productie 
van auto-antilichamen en auto-immuniteit, microvasculopathie en disfunctionerende fibroblasten wat leidt tot overmatige fibrose. Als de darmen zijn aangedaan kan dit leiden tot verminderde motiliteit. Processen die betrokken zijn bij de pathogenese in de darmen zijn microvasculaire schade, disfunctie van autonome zenuwen, musculaire atrofie en fibrose, en auto-antilichamen. Momenteel is het niet duidelijk hoe deze mechanismen op elkaar inwerken. Waarschijnlijk zijn microvasculaire schade en ischemie de belangrijkste gebeurtenissen bij betrokkenheid van de darmen.

In de literatuur wordt beschreven dat ontwikkeling van intestinale dismotiliteit bij SSc een sequentieel proces zou kunnen zijn. Dit proces zou beginnen met vasculaire veranderingen, leidend tot neurale schade, atrofie van de muscularis propria en gevolgd door fibrose. Daarom verwachtten we dat in weefselmateriaal van SSc-patiënten met fibrose in de muscularis propria (het eindstadium van het proces van intestinale dismotiliteit) tevens vasculaire veranderingen en neurale schade waargenomen zou kunnen worden. Onze resultaten konden dit slechts gedeeltelijk bevestigen, wat suggereert dat in plaats van sequentiële veranderingen, meerdere parallelle processen plaatsvinden. Deze verschillende processen zouden de grote variatie in morfologische kenmerken en klinische klachten tussen individuele patiënten kunnen verklaren.

\section{Algemene conclusie}

Tot slot worden de resultaten en conclusies van dit proefschrift in een breder perspectief besproken. De twee opvallendste bevindingen in onze studies naar secundaire motiliteitsstoornissen zijn een afgenomen dichtheid van het myenterische ICC-netwerk en een verminderde dichtheid van gliacellen in een groot deel van de patiëntgroepen. Door ons te beperken tot goed gedefinieerde secundaire motiliteitsstoornissen hebben we gestreefd naar zo uniform mogelijke groepen, in tegenstelling tot de heterogene groep van primaire GINMD's. De studie naar specifieke ziektebeelden heeft nieuwe inzichten en hypotheses binnen het veld van de GINMD's opgeleverd. De verrassende heterogeniteit binnen onze patiëntencohorten maakt het niet goed mogelijk om algemene pathofysiologische mechanismes af te leiden voor (primaire) darmmotiliteitsstoornissen. In de toekomst zal het daarom belangrijk blijven om de neuromusculaire structuren in de darm van elke individuele patiënt met ernstige klinische motiliteitsklachten te beoordelen. Een zorgvuldige histopathologische diagnose zal uiteindelijk bijdragen aan een nauwkeurige diagnose, een betere prognose en beter aansluitende (gerichte) therapie voor de patiënt. 
Nederlandse samenvatting 
Appendices 


\section{List of abbreviations}

$\begin{array}{ll}\text { AA } & \text { Serum amyloid A amyloidosis } \\ \text { AL } & \text { Amyloid light-chain amyloidosis } \\ \text { CIPO } & \text { Chronic intestinal pseudo-obstruction } \\ \text { CL } & \text { Circular layer of muscularis propria } \\ \text { CNS } & \text { Central nervous system } \\ \text { dcSSc } & \text { Diffuse cutaneous form of SSc } \\ \text { ENS } & \text { Enteric nervous system } \\ \text { EVG } & \text { Elastic von Gieson } \\ \text { FFPE } & \text { Formalin-fixed paraffin-embedded } \\ \text { GI } & \text { Gastrointestinal } \\ \text { GINMD } & \text { Gastrointestinal neuromuscular disease } \\ \text { GINMP } & \text { Gastrointestinal neuromuscular pathology } \\ \text { H\&E } & \text { Haematoxylin and Eosin } \\ \text { ICC } & \text { Interstitial cell of Cajal } \\ \text { IPAN } & \text { Intrinsic primary afferent neuron } \\ \text { lcSSc } & \text { Limited cutaneous form of SSc } \\ \text { LL } & \text { Longitudinal layer of muscularis propria } \\ \text { PAS } & \text { Periodic acid Schiff } \\ \text { SB } & \text { Spina bifida } \\ \text { SCI } & \text { Spinal cord injury } \\ \text { SMA } & \text { Smooth muscle actin } \\ \text { SSc } & \text { Systemic sclerosis } \\ \text { STC } & \text { Slow transit constipation } \\ \end{array}$


Appendices 


\section{List of publications}

Den Braber-Ymker M, Heijker S, Lammens M, Nagtegaal ID. Practical and reproducible estimation of myenteric interstitial cells of Cajal in the bowel for diagnostic purposes. Neurogastroenterol Motil, 2016. 28(8): p. 1261-1267.

Den Braber-Ymker M, Lammens M, van Putten MJAM, Nagtegaal ID. The enteric nervous system and the musculature of the colon are altered in patients with spina bifida and spinal cord injury. Virchows Arch, 2017. 470(2): p. 175-184.

Den Braber-Ymker M, Heijker S, Lammens M, Nagtegaal ID. Intestinal involvement in amyloidosis is a sequential process. Neurogastroenterol Motil, 2018. 30(12): p. e13469.

Den Braber-Ymker M, Vonk MC, Grünberg K, Lammens M, Nagtegaal ID. Intestinal hypomotility in systemic sclerosis: a histological study into the sequence of events.

Submitted and under review.

\section{List of abstracts and presentations}

Den Braber-Ymker M, Uijttenboogaart JT, Lammens M, Nagtegaal ID. Histological investigations into intestinal dysmotility in systemic sclerosis.

Poster presentation, FNM 2016 Joint International Meeting: 2nd Federation of Neurogastroenterology and Motility Meeting, San Francisco.

Den Braber-Ymker M, Heijker S, Lammens M, Nagtegaal ID. Amyloid in the intestines: myopathy seems the most likely cause of dysmotility.

Poster presentation, NeuroGASTRO 2017, Cork, Ireland. 
Appendices 


\section{Curriculum Vitae}

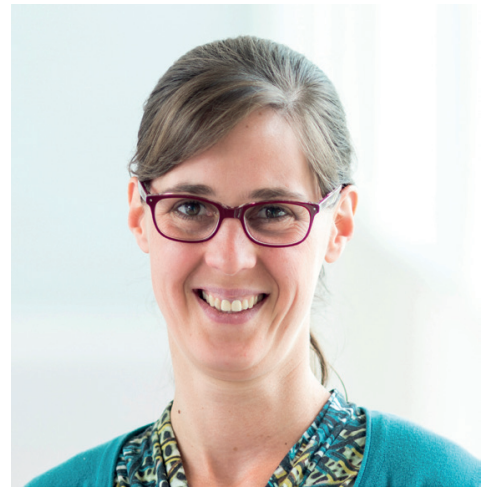

Marjanne den Braber-Ymker werd op 6 mei 1986 geboren te Zweeloo. Zij behaalde haar $\mathrm{VWO}+$ diploma aan het Hondsrugcollege in Emmen in 2004, waarna zij begon aan haar studie Technische Geneeskunde aan de Universiteit Twente. In de zomer van 2006 nam zij deel aan de masterclass Pathologie, waar haar interesse voor pathologie werd gewekt. Door verschillende studentassistentschappen maakte zij kennis met het begeleiden van studenten waardoor haar enthousiasme voor het geven van onderwijs werd aangewakkerd. Tijdens haar master Reconstructive Medicine heeft ze achtereenvolgens stages gelopen op de afdelingen Vaatchirurgie (Medisch Spectrum Twente), Brandwondencentrum (Beverwijk), Interventiecardiologie (Medisch Spectrum Twente) en Hoofd-halsoncologie (Antoni van Leeuwenhoek ziekenhuis). Na het voltooien van haar afstudeerstage bij BIOS (Labon-a-Chip groep, Universiteit Twente) en Medisch Spectrum Twente ontving zij in 2011 haar masterdiploma. In 2012 is zij aan haar promotieonderzoek naar darmmotiliteitsstoornissen begonnen op de afdeling Pathologie van het Radboudumc onder begeleiding van prof. dr. ir. Michel van Putten (Universiteit Twente), prof. dr. Iris Nagtegaal (Radboudumc) en prof. dr. Martin Lammens (Radboudumc/UZ Antwerpen). Zij heeft haar promotieonderzoek parttime gedaan, waarbij ze de andere helft van haar tijd werkte als docent vanuit de afdeling Pathologie. Begin 2017 behaalde zij haar Basiskwalificatie Onderwijs. Momenteel is ze werkzaam als docent op de afdeling Pathologie van het Radboudumc. Als docent verzorgt zij onderwijs bij Technische Geneeskunde (Universiteit Twente) en bij Geneeskunde/Biomedische Wetenschappen (Radboudumc).

Marjanne is in 2012 getrouwd met Ton den Braber en woont in Arnhem. 
Appendices 


\section{Dankwoord}

$\mathrm{Na}$ zeven jaar is het einde van mijn promotietraject aangebroken! Dit had ik niet kunnen doen zonder de hulp en steun van veel mensen om mij heen. Bedankt voor jullie tijd, enthousiasme, hulp en vertrouwen! Een aantal mensen wil ik graag persoonlijk bedanken.

Iris Nagtegaal, beste Iris, bedankt voor je vele ideeën, je enthousiasme en begeleiding in de afgelopen jaren! Ik heb veel van je geleerd, zowel inhoudelijk als de manier waarop ik de resultaten op een goede manier kon weergeven en beschrijven. In de tijd dat alles teveel voor me was, heb je me geholpen om kleine, niet te perfecte stapjes te zetten, waardoor ik weer vertrouwen in mezelf kreeg en vooruitgang boekte. Het was zeker in het begin zoeken naar de richting die we met het onderzoek op wilden en op welke manier we de studies gingen opzetten. Gaandeweg werd dat steeds duidelijker en nu ligt er een proefschrift waar ik trots op ben.

Michel van Putten, beste Michel, ondanks dat je inhoudelijk verder bij mijn onderzoek vandaan stond heb ik veel gehad aan onze evaluatiemomenten, je vertrouwen en aanmoediging om verder te gaan. Soms had ik het gevoel dat ik ver achter lag op schema, maar dan stelde je me gerust met de opmerking dat het bij andere promovendi niet veel anders verliep. Ik vind het jammer dat onze plannen om een pilot te doen met 'EMGmetingen' van de darm niet door zijn gegaan, maar gezien de omstandigheden is het voor mij wel een verstandige keuze geweest. Bedankt voor je tijd en begeleiding!

Martin Lammens, beste Martin, we begonnen samen met Iris enthousiast met het onderzoek. Je hebt me in een half jaar tijd veel geleerd over darmmotiliteitsstoornissen, hoe ik de coupes moest bekijken, welke kleuringen bruikbaar zijn, etc. Toen je naar Antwerpen ging bleef je betrokken bij mijn onderzoek, je hebt veel goede ideeën gegeven en kritische vragen gesteld over de studies die we hebben gedaan. Bedankt daarvoor!

Sanneke Heijker, bedankt voor de samenwerking in de ICC-studie en het onderzoek over amyloïdose. Sandra Croockewit, bedankt voor de samenwerking in de studie over amyloïdose. Madelon Vonk en Katrien Grünberg, bedankt voor de samenwerking in het onderzoek over systemische sclerose.

De studenten die een stukje aan mijn onderzoek hebben bijgedragen: Sarah, Jasper, Frank, Esmée en Bryan. Ik vond het leuk om jullie stages te begeleiden. Bedankt voor jullie inzet. 
In de afgelopen jaren heb ik veel verschillende kamergenoten gehad: Nikki, Bart, Monica, Michiel Simons, Michiel van den Brand, Blanca, Thomas, Diede en Tijmen. Bedankt voor de gezelligheid, een luisterend oor, het delen van hoogtepunten en frustraties.

Peter de Wilde, zonder jou was ik wellicht nooit op de afdeling Pathologie terecht gekomen. Bedankt voor je inzet om een plek te creëren waarin ik mijn promotieonderzoek met onderwijs kon combineren. Ik heb veel van je geleerd. Je passie voor pathologie en wiskunde en je enthousiasme voor het onderwijs waren aanstekelijk. Rob de Waal, we hebben veel samengewerkt als onderwijscollega's. Bedankt voor alles wat ik van je heb geleerd dankzij je jarenlange ervaring.

Alle andere onderwijscollega's uit Nijmegen en Enschede, bedankt voor jullie samenwerking en enthousiasme. Mede dankzij jullie heb ik me kunnen ontwikkelen als docent en ontdekt dat ik het werken als docent bij de opleidingen Geneeskunde/ Biomedische wetenschappen en Technische Geneeskunde ontzettend leuk vind.

Annemieke, we hebben er al heel wat 'lunchkilometers' opzitten waarin we de werkstress even konden loslaten, ons hart konden luchten en elkaar scherp hielden. Ik hoop dat er nog vele kilometers zullen volgen. Bedankt voor je collegialiteit en gezelligheid. Ik ben blij met jou als paranimf?

Else, al zo'n lange tijd vriendinnen en nu bijna tegelijkertijd promoveren. Aan het korte weekje Makkum afgelopen voorjaar heb ik goede herinneringen: schrijven aan ons proefschrift terwijl het buiten stormde was effectief en inspirerend. Bedankt voor je vriendschap en dat je als paranimf naast me staat!

Lieve (schoon)familie en vrienden, jullie hebben je vast vaak afgevraagd wat ik allemaal heb gedaan de afgelopen jaren. Ik hoop dat mijn boekje het een klein beetje duidelijker maakt. Bedankt voor jullie interesse en steun. Erna, dankzij jou heb ik een prachtige kaft om mijn boekje. Bedankt voor de tijd die je daarvoor hebt vrijgemaakt! Mijn schoonouders, pa en ma Den Braber, bedankt voor jullie vertrouwen in mij en dat ik bij jullie altijd welkom ben. Mijn ouders, papa en mama Ymker, bedankt voor jullie liefde, steun en vertrouwen!

Lieve Ton, ik ben heel dankbaar datjij naast me staat in mijn leven. Je relativeringsvermogen helpt me om dingen positief te bekijken. Je weet me vaak op de kast te krijgen en maakt me aan het lachen. Je hebt me geholpen in de periode dat ik overspannen was en me aangemoedigd om mijn promotietraject af te maken. Bedankt voor je liefde en je geduld in de afgelopen jaren! 
Van al Uw goedheid wil ik blijven zingen;

tienduizend redenen tot dankbaarheid!

Opwekking 733 
